\title{
Quantum trigonometric Calogero-Sutherland model, irreducible characters and Clebsch-Gordan series for the exceptional algebra $E_{7}$
}

\author{
J. Fernández Núñez ${ }^{\dagger}$,W. García Fuertes ${ }^{\dagger}$, A.M. Perelomov ${ }^{\ddagger} *$ \\ $\dagger$ Departamento de Física, Facultad de Ciencias, Universidad de Oviedo, E-33007 Oviedo, Spain \\ $\ddagger$ Departamento de Física Teórica, Universidad de Zaragoza, E-50009 Zaragoza, Spain
}

\begin{abstract}
We re-express the quantum Calogero-Sutherland model for the Lie algebra $E_{7}$ and the particular value of the coupling constant $\kappa=1$ by using the fundamental irreducible characters of the algebra as dynamical variables. For that, we need to develop a systematic procedure to obtain all the ClebschGordan series required to perform the change of variables. We describe how the resulting quantum Hamiltonian operator can be used to compute more characters and Clebsch-Gordan series for this exceptional algebra.
\end{abstract}

\section{Introduction}

Integrable systems are important because they can be considered as 0-th order perturbative aproximations to non-integrable systems. By integrability we mean here integrability in the sense of Liouville, that is, the existence of a complete set of mutually commuting integrals of motion. During the three last decades of the past century, a plethora of highly nontrivial (classical and quantum) mechanical integrable systems were discovered, see $[1,2]$ for comprehensive reviews. Among these, the Calogero-Sutherland models form a distinguished class. The first analysis of a system of this kind was performed by Calogero [3] who studied, from the quantum standpoint, the dynamics on the infinite line of a set of particles interacting pairwise by rational plus quadratic potentials, and found that the problem was exactly solvable. Soon afterwards, Sutherland [4] arrived to similar results for the quantum problem on the circle, this time with trigonometric interaction; and later Moser [5] proved, in terms of Lax pairs, that the classical counterparts of these models also enjoyed integrability.

The identification of the general scope of these discoveries came with the work of Olshanetsky and Perelomov $[6,7,8]$, who realized that it is possible to associate models of this kind to all the root systems of the simple Lie algebras, and that all these models are integrable, both in the classical and the quantum framework [9, 10], for interactions of the type rational (or inverse-square), $q^{-2}$; rational+quadratic, $q^{-2}+\omega^{2} q^{2}$; trigonometric, $\sin ^{-2} q$; hyperbolic, $\sinh ^{-2} q$; and the most general, given by the Weierstrass elliptic function $\mathcal{P}(q)$. Nowadays, there is a widespread interest in this kind of integrable systems, and many mathematical and physical applications for them have been found, see for instance [11]. In Physics, we mention, among others, the remarkable connection established $[12,13]$ between the different CalogeroSutherland models and the properties of the equations describing the physics of disordered wires (the DMPK equation); the results are in good agreement with the experimental observations.

The study of the form and properties of the Schrödinger eigenfunctions for the quantum version of these models constitutes by itself an interesting line of research. In fact, these eigenfunctions have very

${ }^{*}$ On leave of absence from the Institute for Theoretical and Experimental Physics, 117259, Moscow, Russia. Current E-mail address: perelomo@mpim-bonn.mpg.de 
rich mathematical properties. In particular, for the trigonometric case, if we tune the coupling constants to some especial values, the wave functions correspond to the characters of the simple Lie algebras, while if we select a different tuning, we can make them to coincide with zonal spherical functions. Thus, the Calogero-Sutherland theories provide us with a new tool for computing these quantities. In this spirit, we will describe in the present paper how to use the trigonometric Calogero-Sutherland model to obtain both particular characters and Clebsch-Gordan series for the exceptional Lie algebra $E_{7}$. The main point of our approach is to express the Hamiltonian in a suitable set of independent variables, indeed the fundamental characters of $E_{7}$. The use of such kind of variables has been quite useful to solve the Schrödinger equation for the models associated to some algebras [6], [14]-[21].

The organization of the paper is as follows. Section 2 is a reminder of the properties of $E_{7}$ relevant for the contents of the paper. Section 3 describes the main properties of the Calogero-Sutherland models associated to root systems and explains how to find the Hamiltonian in the variables mentioned above. Section 4 gives some account of the computation of the Clebsch-Gordan series of $E_{7}$ needed to pass to the new variables. In Section 5 we present the Hamiltonian in these variables and describe its use for computing new characters and to reduce tensor products of representations. Some conclusions are given in Section 6, and finally, the appendices show some explicit results for characters and Clebsch-Gordan series of $E_{7}$.

\section{Summary of results on the Lie algebra $E_{7}$}

In this Section, we review some standard facts about the root and weight systems of the Lie algebra $E_{7}$, with the aim of fixing the notation and help the reader to follow the rest of the paper. More extensive and sound treatments of these topics can be found in many excellent textbooks, see for instance [22, 23].

The complex Lie algebra $E_{7}$ has dimension 133 and rank 7 , as the name suggests. From the geometrical point of view, it admits (with some subtleties, see [24]) an interpretation which extends the standard-one for the classical algebras: in the same way that these correspond to the isometries of projective spaces over the first three normed division algebras $-S O(n+1) \simeq \operatorname{Isom}\left(\mathbf{R} P^{n}\right), S U(n+1) \simeq \operatorname{Isom}\left(\mathbf{C} P^{n}\right)$, $S p(n+1) \simeq \operatorname{Isom}\left(\mathbf{H} P^{n}\right)-F_{4}, E_{6}, E_{7}$ and $E_{8}$ are the Lie algebras of the projective planes over extensions of the octonions, giving rise to the so-called "magic square": $F_{4} \simeq \operatorname{Isom}\left(\mathbf{O} P^{2}\right), E_{6} \simeq \operatorname{Isom}\left[(\mathbf{C} \otimes \mathbf{O}) P^{2}\right]$, $E_{7} \simeq \operatorname{Isom}\left[(\mathbf{H} \otimes \mathbf{O}) P^{2}\right], E_{8} \simeq \operatorname{Isom}\left[(\mathbf{O} \otimes \mathbf{O}) P^{2}\right]$.

The Dynkin diagram of $E_{7}$, see Figure 1 ,

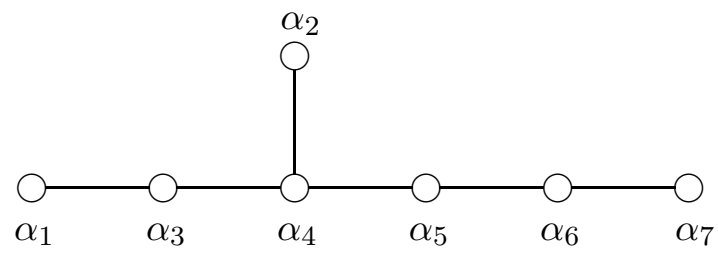

Figure 1. The Dynkin diagram for the Lie algebra $E_{7}$.

encodes the euclidean relations $A_{i j}=\left(\alpha_{i}, \alpha_{j}\right)$ among the simple roots, which are

$$
\begin{aligned}
\left(\alpha_{i}, \alpha_{i}\right) & =2, & & i=1, \ldots, 7 \\
\left(\alpha_{i}, \alpha_{i+2}\right) & =-1, & & i=1,2 \\
\left(\alpha_{i}, \alpha_{i+1}\right) & =-1, & & i=3, \ldots, 6 \\
\left(\alpha_{i}, \alpha_{j}\right) & =0, & & \text { in all other cases. }
\end{aligned}
$$


Therefore, the Cartan matrix $A=\left(A_{i j}\right)$ and its inverse $A^{-1}=\left(A_{i j}^{-1}\right)$ read

$$
A=\left(\begin{array}{ccccccc}
2 & 0 & -1 & 0 & 0 & 0 & 0 \\
0 & 2 & 0 & -1 & 0 & 0 & 0 \\
-1 & 0 & 2 & -1 & 0 & 0 & 0 \\
0 & -1 & -1 & 2 & -1 & 0 & 0 \\
0 & 0 & 0 & -1 & 2 & -1 & 0 \\
0 & 0 & 0 & 0 & -1 & 2 & -1 \\
0 & 0 & 0 & 0 & 0 & -1 & 2
\end{array}\right), \quad A^{-1}=\frac{1}{2}\left(\begin{array}{ccccccc}
4 & 4 & 6 & 8 & 6 & 4 & 2 \\
4 & 7 & 8 & 12 & 9 & 6 & 3 \\
6 & 8 & 12 & 16 & 12 & 8 & 4 \\
8 & 12 & 16 & 24 & 18 & 12 & 6 \\
6 & 9 & 12 & 18 & 15 & 10 & 5 \\
4 & 6 & 8 & 12 & 10 & 8 & 4 \\
2 & 3 & 4 & 6 & 5 & 4 & 3
\end{array}\right) .
$$

Throughout this paper we will use a realization of this root system in terms of a system of vectors $\left\{v_{i}\right\}_{i=1, \ldots, 8}$ of $\mathbf{R}^{8}$ (endowed with the standard Euclidean product $\left.(),\right)$ satisfying the relations $\left(v_{i}, v_{j}\right)=$ $-\frac{1}{8}+\delta_{i j}[22]$. With reference to this system, $E_{7}$ is the root system in the hyperplane $V \subset \mathbf{R}^{8}$ of equation $\sum_{i} v_{i}=0$ given by $\mathcal{R}=\left\{v_{i}-v_{j}, v_{i}+v_{j}+v_{k}+v_{l} \mid i \neq j \neq k \neq l\right\}$, the positive ones being those in the subset $\mathcal{R}^{+}=\left\{v_{i}-v_{j}, v_{8}-v_{i}, v_{i}+v_{j}+v_{k}+v_{8} \mid i<j<k<8\right\}$. There are 63 positive roots, which can be classified by heights as indicated in Table 1 . The seven simple roots are

$$
\begin{array}{ll}
\alpha_{1}=v_{1}-v_{2}, & \alpha_{2}=v_{4}+v_{5}+v_{6}+v_{7}, \\
\alpha_{3}=v_{2}-v_{3}, & \alpha_{4}=v_{3}-v_{4}, \\
\alpha_{5}=v_{4}-v_{5}, & \alpha_{6}=v_{5}-v_{6}, \\
\alpha_{7}=v_{6}-v_{7}, &
\end{array}
$$

which clearly satisfy the relations (1).

The hyperplane $V$ can be viewed as $\mathbf{R}^{7}$, and the basis made with the vectors $v_{1}, \ldots, v_{7}$ is related to the canonical basis $\left\{e_{k}\right\}_{k=1, \ldots, 7}$ by $v_{k}=e_{k}-\frac{1}{7}\left(1+\frac{1}{\sqrt{8}}\right) \sum_{j=1}^{7} e_{j}$; thus, the simple roots $\alpha_{i}$ are given by

$$
\begin{aligned}
\alpha_{1} & =e_{1}-e_{2}, \\
\alpha_{2} & =\frac{1}{7}\left(3-\frac{2}{\sqrt{2}}\right) \sum_{j=4}^{7} e_{j}-\frac{4}{7}\left(1+\frac{1}{\sqrt{8}}\right) \sum_{j=1}^{3} e_{j}, \\
\alpha_{k} & =e_{k-1}-e_{k}, \quad k=3, \ldots, 7 .
\end{aligned}
$$

The fundamental weights $\lambda_{i}=\sum_{j=1}^{7} A_{j i}^{-1} \alpha_{j}$ are

$$
\begin{aligned}
& \lambda_{1}=v_{1}-v_{8}, \\
& \lambda_{2}=-2 v_{8}, \\
& \lambda_{3}=v_{1}+v_{2}-2 v_{8}, \\
& \lambda_{4}=v_{1}+v_{2}+v_{3}+3 v-3 v_{8}, \\
& \lambda_{5}=v_{1}+v_{2}+v_{3}+v_{4}-2 v_{8}, \\
& \lambda_{6}=v_{1}+v_{2}+v_{3}+v_{4}+v_{5}-v_{8}, \\
& \lambda_{7}=v_{1}+v_{2}+v_{3}+v_{4}+v_{5}+v_{6},
\end{aligned}
$$

as it follows from (2) and (3). As $E_{7}$ is simply-laced, the geometry of the weight system is summarized by the relations $\left(\lambda_{i}, \lambda_{j}\right)=A_{i j}^{-1}$. The Weyl vector is

$$
\rho=\frac{1}{2} \sum_{\alpha \in \mathcal{R}^{+}} \alpha=\sum_{i=1}^{7} \lambda_{i}=\frac{1}{2}\left(34 \alpha_{1}+49 \alpha_{2}+66 \alpha_{3}+96 \alpha_{4}+75 \alpha_{5}+52 \alpha_{6}+27 \alpha_{7}\right),
$$




\begin{tabular}{|c|c|}
\hline ht & Positive roots \\
\hline 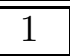 & $\alpha_{1}, \alpha_{2}, \alpha_{3}, \alpha_{4}, \alpha_{5}, \alpha_{6}, \alpha_{7}$ \\
\hline 2 & $\alpha_{1}+\alpha_{3}, \alpha_{3}+\alpha_{4}, \alpha_{4}+\alpha_{5}, \alpha_{5}+\alpha_{6}, \alpha_{2}+\alpha_{4}, \alpha_{6}+\alpha_{7}$ \\
\hline 3 & $\alpha_{1}+\alpha_{3}+\alpha_{4}, \alpha_{3}+\alpha_{4}+\alpha_{5}, \alpha_{4}+\alpha_{5}+\alpha_{6}, \alpha_{2}+\alpha_{3}+\alpha_{4}, \alpha_{2}+\alpha_{4}+\alpha_{5}, \alpha_{5}+\alpha_{6}+\alpha_{7}$ \\
\hline 4 & $\begin{array}{l}\alpha_{1}+\alpha_{3}+\alpha_{4}+\alpha_{5}, \alpha_{3}+\alpha_{4}+\alpha_{5}+\alpha_{6}, \alpha_{1}+\alpha_{2}+\alpha_{3}+\alpha_{4}, \alpha_{2}+\alpha_{3}+\alpha_{4}+\alpha_{5} \\
\alpha_{2}+\alpha_{4}+\alpha_{5}+\alpha_{6}, \alpha_{4}+\alpha_{5}+\alpha_{6}+\alpha_{7}\end{array}$ \\
\hline 5 & $\begin{array}{l}\alpha_{1}+\alpha_{3}+\alpha_{4}+\alpha_{5}+\alpha_{6}, \alpha_{1}+\alpha_{2}+\alpha_{3}+\alpha_{4}+\alpha_{5}, \alpha_{2}+\alpha_{3}+2 \alpha_{4}+\alpha_{5} \\
\alpha_{2}+\alpha_{3}+\alpha_{4}+\alpha_{5}+\alpha_{6}, \alpha_{2}+\alpha_{4}+\alpha_{5}+\alpha_{6}+\alpha_{7}, \alpha_{3}+\alpha_{4}+\alpha_{5}+\alpha_{6}+\alpha_{7}\end{array}$ \\
\hline 6 & $\begin{array}{l}\alpha_{1}+\alpha_{2}+\alpha_{3}+2 \alpha_{4}+\alpha_{5}, \alpha_{1}+\alpha_{2}+\alpha_{3}+\alpha_{4}+\alpha_{5}+\alpha_{6}, \alpha_{2}+\alpha_{3}+2 \alpha_{4}+\alpha_{5}+\alpha_{6}, \\
\alpha_{1}+\alpha_{3}+\alpha_{4}+\alpha_{5}+\alpha_{6}+\alpha_{7}, \alpha_{2}+\alpha_{3}+\alpha_{4}+\alpha_{5}+\alpha_{6}+\alpha_{7}\end{array}$ \\
\hline 7 & $\begin{array}{l}\alpha_{1}+\alpha_{2}+2 \alpha_{3}+2 \alpha_{4}+\alpha_{5}, \alpha_{2}+\alpha_{3}+2 \alpha_{4}+2 \alpha_{5}+\alpha_{6}, \alpha_{1}+\alpha_{2}+\alpha_{3}+2 \alpha_{4}+\alpha_{5}+\alpha_{6}, \\
\alpha_{1}+\alpha_{2}+\alpha_{3}+\alpha_{4}+\alpha_{5}+\alpha_{6}+\alpha_{7}, \alpha_{2}+\alpha_{3}+2 \alpha_{4}+\alpha_{5}+\alpha_{6}+\alpha_{7}\end{array}$ \\
\hline 8 & $\begin{array}{l}\alpha_{1}+\alpha_{2}+2 \alpha_{3}+2 \alpha_{4}+\alpha_{5}+\alpha_{6}, \alpha_{1}+\alpha_{2}+\alpha_{3}+2 \alpha_{4}+2 \alpha_{5}+\alpha_{6} \\
\alpha_{1}+\alpha_{2}+\alpha_{3}+2 \alpha_{4}+\alpha_{5}+\alpha_{6}+\alpha_{7}, \alpha_{2}+\alpha_{3}+2 \alpha_{4}+2 \alpha_{5}+\alpha_{6}+\alpha_{7}\end{array}$ \\
\hline 9 & $\begin{array}{l}\alpha_{1}+\alpha_{2}+2 \alpha_{3}+2 \alpha_{4}+2 \alpha_{5}+\alpha_{6}, \alpha_{1}+\alpha_{2}+\alpha_{3}+2 \alpha_{4}+2 \alpha_{5}+\alpha_{6}+\alpha_{7} \\
\alpha_{1}+\alpha_{2}+2 \alpha_{3}+2 \alpha_{4}+\alpha_{5}+\alpha_{6}+\alpha_{7}, \alpha_{2}+\alpha_{3}+2 \alpha_{4}+2 \alpha_{5}+2 \alpha_{6}+\alpha_{7}\end{array}$ \\
\hline 10 & $\begin{array}{l}\alpha_{1}+\alpha_{2}+\alpha_{3}+2 \alpha_{4}+2 \alpha_{5}+2 \alpha_{6}+\alpha_{7}, \alpha_{1}+\alpha_{2}+2 \alpha_{3}+2 \alpha_{4}+2 \alpha_{5}+\alpha_{6}+\alpha_{7}, \\
\alpha_{1}+\alpha_{2}+2 \alpha_{3}+3 \alpha_{4}+2 \alpha_{5}+\alpha_{6}\end{array}$ \\
\hline 11 & $\begin{array}{l}\alpha_{1}+2 \alpha_{2}+2 \alpha_{3}+3 \alpha_{4}+2 \alpha_{5}+\alpha_{6}, \alpha_{1}+\alpha_{2}+2 \alpha_{3}+2 \alpha_{4}+2 \alpha_{5}+2 \alpha_{6}+\alpha_{7} \\
\alpha_{1}+\alpha_{2}+2 \alpha_{3}+3 \alpha_{4}+2 \alpha_{5}+\alpha_{6}+\alpha_{7}\end{array}$ \\
\hline 12 & $\alpha_{1}+\alpha_{2}+2 \alpha_{3}+3 \alpha_{4}+2 \alpha_{5}+2 \alpha_{6}+\alpha_{7}, \alpha_{1}+2 \alpha_{2}+2 \alpha_{3}+3 \alpha_{4}+2 \alpha_{5}+\alpha_{6}+\alpha_{7}$ \\
\hline 13 & $\alpha_{1}+\alpha_{2}+2 \alpha_{3}+3 \alpha_{4}+3 \alpha_{5}+2 \alpha_{6}+\alpha_{7}, \alpha_{1}+2 \alpha_{2}+2 \alpha_{3}+3 \alpha_{4}+2 \alpha_{5}+2 \alpha_{6}+\alpha_{7}$ \\
\hline 14 & $\alpha_{1}+2 \alpha_{2}+2 \alpha_{3}+3 \alpha_{4}+3 \alpha_{5}+2 \alpha_{6}+\alpha_{7}$ \\
\hline 15 & $\alpha_{1}+2 \alpha_{2}+2 \alpha_{3}+4 \alpha_{4}+3 \alpha_{5}+2 \alpha_{6}+\alpha_{7}$ \\
\hline 16 & $\alpha_{1}+2 \alpha_{2}+3 \alpha_{3}+4 \alpha_{4}+3 \alpha_{5}+2 \alpha_{6}+\alpha_{7}$ \\
\hline 17 & $2 \alpha_{1}+2 \alpha_{2}+3 \alpha_{3}+4 \alpha_{4}+3 \alpha_{5}+2 \alpha_{6}+\alpha_{7}$ \\
\hline
\end{tabular}

Table 1: Heights of positive roots of $E_{7}$.

and has lenght $|\rho|=\sqrt{798} / 2$. The Weyl formula for dimensions applied to the irreducible representation associated to the integral dominant weight $\mu=\sum_{i=1}^{7} m_{i} \lambda_{i}$ gives

$$
\operatorname{dim} R_{\mu}=\prod_{\alpha \in \mathcal{R}^{+}} \frac{(\alpha, \mu+\rho)}{(\alpha, \rho)}=\frac{P}{2^{6} \cdot 3^{6} \cdot 4^{6} \cdot 5^{6} \cdot 6^{5} \cdot 7^{5} \cdot 8^{4} \cdot 9^{4} \cdot 10^{3} \cdot 11^{3} \cdot 12^{2} \cdot 13^{2} \cdot 14 \cdot 15 \cdot 16 \cdot 17}
$$

where $P$ is a product extended to the set of positive roots in which the root $\alpha=\sum_{i=1}^{7} a_{i} \alpha_{i}$ contributes with a factor ht $(\alpha)+\sum_{i=1}^{7} a_{i} m_{i}$, where ht $(\alpha)$ is the height of $\alpha$. In particular, for the basic representations $R_{\lambda_{k}}$, one finds:

$$
\begin{array}{ll}
\operatorname{dim} R_{\lambda_{1}}=133, & \operatorname{dim} R_{\lambda_{2}}=912, \\
\operatorname{dim} R_{\lambda_{3}}=8645, & \operatorname{dim} R_{\lambda_{4}}=365750, \\
\operatorname{dim} R_{\lambda_{5}}=27664, & \operatorname{dim} R_{\lambda_{6}}=1539, \\
\operatorname{dim} R_{\lambda_{7}}=56 . &
\end{array}
$$

All the irreducible representations are self-adjoint; $R_{\lambda_{1}}$ is the adjoint representation and $R_{\lambda_{7}}$, the fundamental one. 


\section{The trigonometric Calogero-Sutherland model associated to a root system}

First of all, we review briefly the general theory of the quantum trigonometric Calogero-Sutherland model related to a root system $\mathcal{R}$ associated to a simple Lie algebra $L$ of rank $r$, and later study explicity the $E_{7}$ case. For Calogero-Sutherland systems other than trigonometric see [10]; see also [25].

The trigonometric Calogero-Sutherland model related to the root system $\mathcal{R}$ of rank $r$ is the quantum system in an Euclidean space $\mathbf{R}^{r}$ defined by the standard Hamiltonian operator

$$
H=\frac{1}{2} \sum_{j=1}^{r} p_{j}^{2}+\sum_{\alpha \in \mathcal{R}^{+}} \kappa_{\alpha}\left(\kappa_{\alpha}-1\right) \sin ^{-2}(\alpha, q),
$$

where $q=\left(q_{j}\right)$ is a cartesian coordinate system and $p_{j}=-\mathrm{i} \partial_{q_{j}} ; \mathcal{R}^{+}$is the set of the positive roots of $L$, and the coupling constants $\kappa_{\alpha}$ are such that $\kappa_{\alpha}=\kappa_{\beta}$ if $|\alpha|=|\beta|$. We will restrict ourselves to the case of simply-laced root systems (as the $E$-series is), for which the Calogero-Sutherland model depends only on one coupling constant $\kappa$.

Although the Hamiltonian (5) is defined in all $\mathbf{R}^{r}$, the configuration space is confined by the singularities (infinite walls) $(\alpha, q)=0$. If the $q$-coordinates are assumed to take values in the $[0, \pi]$ interval, $H$ can be interpreted as describing the dynamics of a system of $r$ unit mass particles moving on the circle with interaction $V(q)=\kappa(\kappa-1) \sum_{\alpha} \sin ^{-2}(\alpha, q)$, but notice that there is not translational invariance. The wave functions have to be $\pi$-periodic.

The main problem is to find the stationary states, i.e., to solve the Schrödinger eigenvalue problem $H \Psi=E \Psi$. The following important facts about this family of quantum mechanical systems were well established in $[6,10]$.

(a)They are integrable, and moreover they are exactly solvable. The configuration space is confined to the Weyl alcove $\Lambda_{W}=\left\{q \in \mathbf{R}^{r} \mid 0<(\alpha, q)<\pi\right\}$.

(b)The ground state energy and (non-normalized) wave function are

$$
\begin{aligned}
& E_{0}(\kappa)=2 \rho^{2} \kappa^{2} \\
& \Psi_{0}^{\kappa}(q)=\prod_{\alpha \in \mathcal{R}^{+}} \sin ^{\kappa}(\alpha, q),
\end{aligned}
$$

while the excited states are indexed by the highest weights $\mu=\sum m_{i} \lambda_{i} \in P^{+}\left(P^{+}\right.$is the cone of dominant weights) of the irreducible representations of $L$, that is, by the $r$-tuple of non-negative integers $\mathbf{m}=\left(m_{1}, \ldots, m_{r}\right)$ (the quantum numbers), and the wave functions satisfy

$$
\begin{aligned}
H \Psi_{\mathbf{m}}^{\kappa} & =E_{\mathbf{m}}(\kappa) \Psi_{\mathbf{m}}^{\kappa} \\
E_{\mathbf{m}}(\kappa) & =2(\mu+\kappa \rho, \mu+\kappa \rho) .
\end{aligned}
$$

(c)It is natural to look for the solutions $\Psi_{\mathbf{m}}^{\kappa}$ in the form

$$
\Psi_{\mathbf{m}}^{\kappa}(q)=\Psi_{0}^{\kappa}(q) \Phi_{\mathbf{m}}^{\kappa}(q),
$$

and consequently we are led to the eigenvalue problem

$$
\Delta^{\kappa} \Phi_{\mathbf{m}}^{\kappa}=\varepsilon_{\mathbf{m}}(\kappa) \Phi_{\mathbf{m}}^{\kappa}
$$

where $\Delta^{\kappa}$ is the linear differential operator

$$
\Delta^{\kappa}=-\frac{1}{2} \sum_{j=1}^{r} \partial_{q_{j}}^{2}-\kappa \sum_{\alpha \in \mathcal{R}^{+}} \cot (\alpha, q)\left(\alpha, \nabla_{q}\right),
$$


and the eigenvalues $\varepsilon_{\mathbf{m}}(\kappa)$ are the energies over the ground level, i.e.,

$$
\varepsilon_{\mathbf{m}}(\kappa)=E_{\mathbf{m}}(\kappa)-E_{0}(\kappa)=2(\mu, \mu+2 \kappa \rho) .
$$

Taking into account that $\left(\lambda_{j}, \lambda_{k}\right)=A_{j k}^{-1}$, it is possible to give a more explicit expression for the eigenvalues $\varepsilon_{\mathbf{m}}(\kappa)$ :

$$
\varepsilon_{\mathbf{m}}(\kappa)=2 \sum_{j, k=1}^{r} A_{j k}^{-1} m_{j} m_{k}+4 \kappa \sum_{j, k=1}^{r} A_{j k}^{-1} m_{j} .
$$

We will write $\varepsilon_{j}(\kappa)$ for the fundamental weigth $\lambda_{j}$, i.e., for the quantum numbers $(0, \ldots, \stackrel{(j)}{1}, \ldots, 0)$

(d)In the case $\kappa=0$ the wave functions (8) are (proportional to) the monomial symmetric functions

$$
M_{\lambda}(q)=\sum_{w \in W} e^{2 i(w \cdot \lambda, q)}, \lambda \in P^{+},
$$

$W$ being the Weyl group of $L$. And the wave functions in the case $\kappa=1$ are (proportional to) the characters of the irreducible representations

$$
\chi_{\lambda}(q)=\frac{\sum_{w \in W}(\operatorname{det} w) e^{2 i(w \cdot(\lambda+\rho), q)}}{\sum_{w \in W}(\operatorname{det} w) e^{2 i(w \cdot \rho, q)}}, \lambda \in P^{+} .
$$

Both $M_{\lambda}$ and $\chi_{\lambda}$ are sums over the orbit of $\lambda$ under $W$, and consequently, $W$-invariant; as wave functions, they represent superpositions of plane waves whose momenta are consistent with the required $\pi$-periodicity.

(d)Due to the Weyl symmetry of the Hamiltonian, the wave functions $\Phi_{\mathbf{m}}^{\kappa}(q)$ are $W$-invariant, and the best way to solve the eigenvalue problem (8) is to use the set of independent $W$-invariant variables $z_{k}=\chi_{\lambda_{k}}(q)$, in terms of which the wave functions $\Phi_{\mathbf{m}}^{\kappa}$ are polynomials.

Unfortunately, the expression of these characters $z_{k}$ in terms of the $q$-variables is complicated and makes the direct change of variables $z=z(q)$ very cumbersome. We are thus forced to follow a much more convenient, indirect route, which has proven to be useful for other root systems, [20, 21].

To this goal, the starting point is to write the operator $\Delta^{\kappa}$ in the $z$-variables:

$$
\Delta^{\kappa}=\sum_{j, k} a_{j k}(z) \partial_{z_{j}} \partial_{z_{k}}+\sum_{j}\left[b_{j}^{0}(z)+\kappa b_{j}^{1}(z)\right] \partial_{z_{j}},
$$

with $a_{j k}=a_{k j}$. Now, if we take into account the fact that, as pointed above, $b_{j}^{0}(z)+b_{j}^{1}(z)=\Delta^{1} z_{j}=$ $\varepsilon_{j}(1) z_{j}$, the full expression for the coefficients $b_{j}(q)=b_{j}^{0}(z)+b_{j}^{1}(z)$ appearing in $\Delta^{1}$ is completely determined by the Cartan matrix of the algebra; explicitly

$$
b_{j}(z)=2\left(A_{j j}^{-1}+2 \sum_{k} A_{k j}^{-1}\right) z_{j}, j=1, \ldots, r .
$$

On the other hand, in order to find the coefficients $a_{j k}$ we will relay on the quadratic Clebsh-Gordan series

$$
R_{\lambda_{j}} \otimes R_{\lambda_{k}}=\sum_{\mu \in Q_{j k}} N_{\mu ; j k} R_{\mu}
$$

where $Q_{j k} \subset P^{+}$is the set of dominant weights in the irreducible representation of highest weight $\lambda_{j}+\lambda_{k}$, and $N_{\lambda ; j k}$ is the multiplicity of the irreducible representation $R_{\lambda}$ in that series; in particular, $N_{\lambda_{j}+\lambda_{k} ; j k}=1$. In these expressions we will write $\mathbf{m}$ or $\left(m_{1}, \ldots, m_{r}\right)$ instead of $\mu=\sum_{i} m_{i} \lambda_{i}$. The Clebsh-Gordan series (16) yield the formulas

$$
z_{j} z_{k}=\sum_{\mathbf{m} \in Q_{j k}} N_{\mathbf{m} ; j k} \chi_{\mathbf{m}}(z)
$$


for the products of fundamental characters $z_{j} z_{k}$, and consequently we obtain the coefficients $a_{j k}$ by applying the operator $\Delta^{1}$ to the two members of (17):

$$
2 a_{j k}(z)=\sum_{\mathbf{m} \in Q_{j k}} N_{\mathbf{m} ; j k} \varepsilon_{\mathbf{m}}(1) \chi_{\mathbf{m}}(z)-b_{j}(z) z_{k}-b_{k}(z) z_{j}, j, k=1, \ldots, r .
$$

Therefore, to accomplish the task of fixing the form of the coefficents $a_{j k}$ we need the list of all the quadratic Clebsh-Gordan series, the explicit expressions of the characters entering in them, and the coefficients $b_{j}$. Although there are some results for $E_{7}$ already available in the literature [22, 26], most of the required Clebsch-Gordan series and characters remain, to our knowledge, to be calculated.

The remaining step to achieve the complete expression of $\Delta^{\kappa}$ is to look for the coefficients $b_{j}^{0}(z)$. These can be found if we know enough monomial symmetric functions $M_{\lambda}$ in terms of the $z$-variables. Suppose that the relations $M_{k}=M_{k}(z)$ are known, where $M_{k}=M_{\lambda_{k}}, k=1, \ldots, r$; then, from the eigenvalue equation $\Delta^{0} M_{k}=\varepsilon_{k}(0) M_{k}$ we obtain the following linear system for the $b^{0}$ 's:

$$
\sum_{i, j} a_{i j}(z) \frac{\partial^{2} M_{k}}{\partial z_{i} \partial z_{j}}+\sum_{j} b_{j}^{0}(z) \frac{\partial M_{k}}{\partial z_{j}}=2 \lambda_{k}^{2} M_{k}(z) .
$$

This system has a unique solution $\left(b_{j}^{0}\right)$ because each of the sets of characters and monomial symmetric functions constitutes a basis of $W$-invariant functions.

Recently [27] we have sound how to find the functions $M_{k}(z)$ in the $E_{6}$ case. In the present paper we will study only the case $\kappa=1$ and consequently we do not need to calculate the $b^{0}$-coefficients now.

\section{The quadratic Clebsh-Gordan series for $E_{7}$}

We have developed a systematic strategy, entirely based in a few elementary facts, to obtain all quantities needed for application of the formula (18). This strategy, which is essentially the same used in the previous paper [21] for the case of $E_{6}$, was described there in full detail, so we will confine ourselves here to mention some very general but important points. First of all, the series should be computed starting from those involving the most external dots of the Dynkin diagram, and going gradually towards the center of it. This is the order that allows the most efficient use of the orthogonality relations. Second, the orthogonality relations should be used not only to fix the multiplicity of some of the weights of lower height, but also to determine linear equations among the multiplicities of several weights of intermediate height. While for $E_{6}$ this is not of great importance, for the more complicated case of $E_{7}$ an extensive use of such linear constraints is required. This constraints, along with the bounds on multiplicities established in [28], make it posible to write a system of diophantine equations with unique solution for these multiplicities. Finally, once all the series are found, the inversion of them to obtain the second-order characters appearing in (18) requires the computation of many other characters of third, fourth and fifth order. The best way to perform these computations is as follows. Starting from the outer region of the Dynkin diagram, we build in each step the part of the $\Delta^{1}$ operator which only requires the characters that we already know. Then, we can use one of the the procedures described in Section 5 below to compute the characters needed to obtain the next coefficient through (18), and so on. This is possible because (18) gives each coefficient $a_{i j}(z)$ in terms of characters associated to weights whose height is lower or equal than $\lambda_{i}+\lambda_{j}$.

By means of these techniques, one finally arrives to the following list of Clebsch-Gordan series:

$$
\begin{aligned}
& R_{\lambda_{1}} \otimes R_{\lambda_{1}}=R_{2 \lambda_{1}} \oplus R_{\lambda_{6}} \oplus R_{\lambda_{3}} \oplus R_{\lambda_{1}} \oplus 1, \\
& R_{\lambda_{1}} \otimes R_{\lambda_{2}}=R_{\lambda_{1}+\lambda_{2}} \oplus R_{\lambda_{7}} \oplus R_{\lambda_{2}} \oplus R_{\lambda_{1}+\lambda_{7}} \oplus R_{\lambda_{5}}, \\
& R_{\lambda_{1}} \otimes R_{\lambda_{3}}=R_{\lambda_{1}+\lambda_{3}} \oplus R_{\lambda_{4}} \oplus R_{\lambda_{1}} \oplus R_{\lambda_{6}} \oplus R_{\lambda_{3}} \oplus R_{2 \lambda_{1}} \oplus R_{\lambda_{1}+\lambda_{6}} \oplus R_{\lambda_{2}+\lambda_{7}},
\end{aligned}
$$




$$
\begin{aligned}
& R_{\lambda_{1}} \otimes R_{\lambda_{4}}=R_{\lambda_{1}+\lambda_{4}} \oplus R_{\lambda_{2}+\lambda_{5}} \oplus R_{\lambda_{3}+\lambda_{6}} \oplus R_{\lambda_{1}+\lambda_{2}+\lambda_{7}} \oplus R_{\lambda_{5}+\lambda_{7}} \oplus R_{2 \lambda_{2}} \oplus R_{\lambda_{1}+\lambda_{3}} \oplus R_{\lambda_{4}} \oplus R_{\lambda_{1}+\lambda_{6}} \\
& \oplus \quad R_{\lambda_{2}+\lambda_{7}} \oplus R_{\lambda_{3}} \\
& R_{\lambda_{1}} \otimes R_{\lambda_{5}}=R_{\lambda_{1}+\lambda_{5}} \oplus R_{\lambda_{2}+\lambda_{6}} \oplus R_{\lambda_{2}} \oplus R_{\lambda_{5}} \oplus R_{\lambda_{1}+\lambda_{2}} \oplus R_{\lambda_{1}+\lambda_{7}} \oplus R_{\lambda_{3}+\lambda_{7}} \oplus R_{\lambda_{6}+\lambda_{7}} \\
& R_{\lambda_{1}} \otimes R_{\lambda_{6}}=R_{\lambda_{1}+\lambda_{6}} \oplus R_{\lambda_{2}+\lambda_{7}} \oplus R_{\lambda_{3}} \oplus R_{\lambda_{6}} \oplus R_{2 \lambda_{7}} \oplus R_{\lambda_{1}} \\
& R_{\lambda_{1}} \otimes R_{\lambda_{7}}=R_{\lambda_{1}+\lambda_{7}} \oplus R_{\lambda_{2}} \oplus R_{\lambda_{7}} \\
& R_{\lambda_{2}} \otimes R_{\lambda_{2}}=R_{2 \lambda_{2}} \oplus R_{\lambda_{1}} \oplus R_{2 \lambda_{7}} \oplus R_{\lambda_{6}} \oplus R_{2 \lambda_{1}} \oplus R_{\lambda_{3}} \oplus R_{\lambda_{2}+\lambda_{7}} \oplus R_{\lambda_{1}+\lambda_{6}} \oplus R_{\lambda_{4}} \oplus 1 \\
& R_{\lambda_{2}} \otimes R_{\lambda_{3}}=R_{\lambda_{2}+\lambda_{3}} \oplus R_{\lambda_{1}+\lambda_{5}} \oplus R_{\lambda_{2}} \oplus R_{\lambda_{5}} \oplus R_{\lambda_{7}} \oplus R_{\lambda_{2}+\lambda_{6}} \oplus R_{\lambda_{3}+\lambda_{7}} \oplus R_{\lambda_{6}+\lambda_{7}} \oplus 2 R_{\lambda_{1}+\lambda_{7}} \\
& \oplus 2 R_{\lambda_{1}+\lambda_{2}} \oplus R_{2 \lambda_{1}+\lambda_{7}} \\
& R_{\lambda_{2}} \otimes R_{\lambda_{4}}=R_{\lambda_{2}+\lambda_{4}} \oplus R_{\lambda_{3}+\lambda_{5}} \oplus R_{\lambda_{1}+\lambda_{2}+\lambda_{6}} \oplus R_{2 \lambda_{2}+\lambda_{7}} \oplus R_{\lambda_{1}+\lambda_{3}+\lambda_{7}} \oplus R_{\lambda_{5}+\lambda_{6}} \oplus R_{\lambda_{4}+\lambda_{7}} \oplus R_{\lambda_{1}+\lambda_{6}+\lambda_{7}} \\
& \oplus \quad R_{2 \lambda_{1}+\lambda_{2}} \oplus 2 R_{\lambda_{2}+\lambda_{3}} \oplus R_{\lambda_{2}+2 \lambda_{7}} \oplus 2 R_{\lambda_{1}+\lambda_{5}} \oplus R_{2 \lambda_{1}+\lambda_{7}} \oplus 2 R_{\lambda_{2}+\lambda_{6}} \oplus 2 R_{\lambda_{3}+\lambda_{7}} \\
& \oplus \quad 2 R_{\lambda_{1}+\lambda_{2}} \oplus R_{\lambda_{6}+\lambda_{7}} \oplus R_{\lambda_{5}} \oplus R_{\lambda_{1}+\lambda_{7}} \oplus R_{\lambda_{2}} \\
& R_{\lambda_{2}} \otimes R_{\lambda_{5}}=R_{\lambda_{2}+\lambda_{5}} \oplus R_{\lambda_{3}+\lambda_{6}} \oplus R_{2 \lambda_{6}} \oplus R_{\lambda_{1}+\lambda_{2}+\lambda_{7}} \oplus R_{2 \lambda_{2}} \oplus R_{\lambda_{1}+\lambda_{3}} \oplus R_{\lambda_{5}+\lambda_{7}} \oplus R_{\lambda_{4}} \oplus R_{\lambda_{1}+2 \lambda_{7}} \\
& \oplus \quad 2 R_{\lambda_{1}+\lambda_{6}} \oplus 2 R_{\lambda_{2}+\lambda_{7}} \oplus R_{2 \lambda_{7}} \oplus R_{2 \lambda_{1}} \oplus R_{\lambda_{3}} \oplus R_{\lambda_{6}} \oplus R_{\lambda_{1}} \\
& R_{\lambda_{2}} \otimes R_{\lambda_{6}}=R_{\lambda_{7}} \oplus R_{\lambda_{2}} \oplus 2 R_{\lambda_{1}+\lambda_{7}} \oplus R_{\lambda_{5}} \oplus R_{\lambda_{6}+\lambda_{7}} \oplus R_{\lambda_{1}+\lambda_{2}} \oplus R_{\lambda_{3}+\lambda_{7}} \oplus R_{\lambda_{2}+\lambda_{6}} \\
& R_{\lambda_{2}} \otimes R_{\lambda_{7}}=R_{\lambda_{2}+\lambda_{7}} \oplus R_{\lambda_{1}} \oplus R_{\lambda_{3}} \oplus R_{\lambda_{6}} \\
& R_{\lambda_{3}} \otimes R_{\lambda_{3}}=R_{2 \lambda_{3}} \oplus R_{\lambda_{1}+\lambda_{4}} \oplus R_{\lambda_{2}+\lambda_{5}} \oplus R_{2 \lambda_{1}+\lambda_{6}} \oplus R_{\lambda_{3}+\lambda_{6}} \oplus 2 R_{\lambda_{1}+\lambda_{2}+\lambda_{7}} \oplus R_{2 \lambda_{6}} \oplus R_{\lambda_{5}+\lambda_{7}} \oplus R_{3 \lambda_{1}} \\
& \oplus \quad R_{2 \lambda_{2}} \oplus 2 R_{\lambda_{1}+\lambda_{3}} \oplus R_{\lambda_{1}+2 \lambda_{7}} \oplus 2 R_{\lambda_{4}} \oplus 3 R_{\lambda_{1}+\lambda_{6}} \oplus 2 R_{2 \lambda_{1}} \oplus 2 R_{\lambda_{2}+\lambda_{7}} \oplus R_{2 \lambda_{7}} \oplus 2 R_{\lambda_{3}} \\
& \oplus \quad 2 R_{\lambda_{6}} \oplus R_{\lambda_{1}} \oplus 1 \\
& R_{\lambda_{3}} \otimes R_{\lambda_{4}}=R_{\lambda_{3}+\lambda_{4}} \oplus R_{\lambda_{1}+\lambda_{2}+\lambda_{5}} \oplus R_{2 \lambda_{2}+\lambda_{6}} \oplus R_{\lambda_{1}+\lambda_{3}+\lambda_{6}} \oplus R_{2 \lambda_{5}} \oplus R_{\lambda_{4}+\lambda_{6}} \\
& \oplus \quad R_{2 \lambda_{1}+\lambda_{2}+\lambda_{7}} \oplus R_{\lambda_{1}+2 \lambda_{6}} \oplus 2 R_{\lambda_{2}+\lambda_{3}+\lambda_{7}} \oplus 2 R_{\lambda_{1}+\lambda_{5}+\lambda_{7}} \oplus R_{2 \lambda_{1}+\lambda_{3}} \oplus R_{2 \lambda_{1}+2 \lambda_{7}} \\
& \oplus 2 R_{\lambda_{1}+2 \lambda_{2}} \oplus R_{2 \lambda_{3}} \oplus 3 R_{\lambda_{1}+\lambda_{4}} \oplus 2 R_{\lambda_{2}+\lambda_{6}+\lambda_{7}} \oplus 2 R_{2 \lambda_{1}+\lambda_{6}} \oplus 3 R_{\lambda_{2}+\lambda_{5}} \oplus R_{\lambda_{3}+2 \lambda_{7}} \oplus 4 R_{\lambda_{3}+\lambda_{6}} \\
& \oplus \quad R_{\lambda_{6}+2 \lambda_{7}} \oplus 5 R_{\lambda_{1}+\lambda_{2}+\lambda_{7}} \oplus R_{2 \lambda_{6}} \oplus 2 R_{2 \lambda_{2}} \oplus 3 R_{\lambda_{5}+\lambda_{7}} \oplus R_{3 \lambda_{1}} \oplus 3 R_{\lambda_{1}+\lambda_{3}} \oplus 2 R_{\lambda_{1}+2 \lambda_{7}} \oplus 3 R_{\lambda_{4}} \\
& \oplus \quad 4 R_{\lambda_{1}+\lambda_{6}} \oplus R_{2 \lambda_{1}} \oplus 3 R_{\lambda_{2}+\lambda_{7}} \oplus R_{2 \lambda_{7}} \oplus 2 R_{\lambda_{3}} \oplus R_{\lambda_{6}} \oplus R_{\lambda_{1}} \\
& R_{\lambda_{3}} \otimes R_{\lambda_{5}}=R_{\lambda_{3}+\lambda_{5}} \oplus R_{\lambda_{1}+\lambda_{2}+\lambda_{6}} \oplus R_{2 \lambda_{2}+\lambda_{7}} \oplus R_{\lambda_{1}+\lambda_{3}+\lambda_{7}} \oplus R_{\lambda_{5}+\lambda_{6}} \oplus R_{\lambda_{4}+\lambda_{7}} \oplus 2 R_{\lambda_{1}+\lambda_{6}+\lambda_{7}} \\
& \oplus \quad R_{2 \lambda_{1}+\lambda_{2}} \oplus R_{\lambda_{2}+2 \lambda_{7}} \oplus 2 R_{\lambda_{2}+\lambda_{3}} \oplus 2 R_{\lambda_{1}+\lambda_{5}} \oplus R_{3 \lambda_{7}} \oplus 2 R_{2 \lambda_{1}+\lambda_{7}} \oplus 3 R_{\lambda_{2}+\lambda_{6}} \oplus 3 R_{\lambda_{3}+\lambda_{7}} \\
& \oplus \quad 3 R_{\lambda_{1}+\lambda_{2}} \oplus 2 R_{\lambda_{6}+\lambda_{7}} \oplus 2 R_{\lambda_{5}} \oplus 3 R_{\lambda_{1}+\lambda_{7}} \oplus R_{\lambda_{2}} \oplus R_{\lambda_{7}} \\
& R_{\lambda_{3}} \otimes R_{\lambda_{6}}=R_{\lambda_{3}+\lambda_{6}} \oplus R_{\lambda_{1}+\lambda_{2}+\lambda_{7}} \oplus R_{2 \lambda_{2}} \oplus R_{\lambda_{1}+\lambda_{3}} \oplus R_{\lambda_{5}+\lambda_{7}} \oplus R_{\lambda_{4}} \oplus R_{\lambda_{1}+2 \lambda_{7}} \oplus 2 R_{\lambda_{1}+\lambda_{6}} \oplus 2 R_{\lambda_{2}+\lambda_{7}} \\
& \oplus \quad R_{2 \lambda_{1}} \oplus R_{2 \lambda_{7}} \oplus 2 R_{\lambda_{3}} \oplus R_{\lambda_{6}} \oplus R_{\lambda_{1}} \\
& R_{\lambda_{3}} \otimes R_{\lambda_{7}}=R_{\lambda_{3}+\lambda_{7}} \oplus R_{\lambda_{1}+\lambda_{7}} \oplus R_{\lambda_{1}+\lambda_{2}} \oplus R_{\lambda_{2}} \oplus R_{\lambda_{5}} \\
& R_{\lambda_{4}} \otimes R_{\lambda_{4}}=R_{2 \lambda_{4}} \oplus R_{\lambda_{2}+\lambda_{3}+\lambda_{5}} \oplus R_{2 \lambda_{3}+\lambda_{6}} \oplus R_{\lambda_{1}+2 \lambda_{5}} \oplus R_{\lambda_{1}+2 \lambda_{2}+\lambda_{6}} \oplus R_{3 \lambda_{2}+\lambda_{7}} \oplus R_{\lambda_{1}+\lambda_{4}+\lambda_{6}} \\
& \oplus 2 R_{\lambda_{1}+\lambda_{2}+\lambda_{3}+\lambda_{7}} \oplus 2 R_{\lambda_{2}+\lambda_{5}+\lambda_{6}} \oplus 2 R_{\lambda_{2}+\lambda_{4}+\lambda_{7}} \oplus R_{2 \lambda_{1}+2 \lambda_{6}} \oplus R_{2 \lambda_{1}+2 \lambda_{2}} \oplus R_{\lambda_{3}+2 \lambda_{6}} \\
& \oplus \quad R_{2 \lambda_{1}+\lambda_{5}+\lambda_{7}} \oplus 3 R_{\lambda_{3}+\lambda_{5}+\lambda_{7}} \oplus R_{\lambda_{1}+2 \lambda_{3}} \oplus 2 R_{2 \lambda_{1}+\lambda_{4}} \oplus 4 R_{\lambda_{1}+\lambda_{2}+\lambda_{6}+\lambda_{7}} \oplus R_{3 \lambda_{6}} \oplus 3 R_{2 \lambda_{2}+\lambda_{3}} \\
& \oplus \quad R_{3 \lambda_{1}+2 \lambda_{7}} \oplus 2 R_{\lambda_{5}+\lambda_{6}+\lambda_{7}} \oplus 2 R_{2 \lambda_{2}+2 \lambda_{7}} \oplus 2 R_{\lambda_{1}+\lambda_{3}+2 \lambda_{7}} \oplus 3 R_{\lambda_{3}+\lambda_{4}} \oplus 6 R_{\lambda_{1}+\lambda_{2}+\lambda_{5}} \\
& \oplus \quad 4 R_{2 \lambda_{2}+\lambda_{6}} \oplus 2 R_{\lambda_{4}+2 \lambda_{7}} \oplus 3 R_{\lambda_{1}+\lambda_{6}+2 \lambda_{7}} \oplus R_{3 \lambda_{1}+\lambda_{6}} \oplus 2 R_{2 \lambda_{5}} \oplus 6 R_{\lambda_{1}+\lambda_{3}+\lambda_{6}} \oplus 6 R_{2 \lambda_{1}+\lambda_{2}+\lambda_{7}} \\
& \oplus 7 R_{\lambda_{4}+\lambda_{6}} \oplus R_{4 \lambda_{1}} \oplus R_{\lambda_{2}+3 \lambda_{7}} \oplus 4 R_{\lambda_{1}+2 \lambda_{6}} \oplus 9 R_{\lambda_{2}+\lambda_{3}+\lambda_{7}} \oplus 9 R_{\lambda_{1}+\lambda_{5}+\lambda_{7}} \oplus 3 R_{2 \lambda_{1}+\lambda_{3}} \\
& \oplus \quad 6 R_{\lambda_{1}+2 \lambda_{2}} \oplus 4 R_{2 \lambda_{1}+2 \lambda_{7}} \oplus 4 R_{2 \lambda_{3}} \oplus 8 R_{\lambda_{2}+\lambda_{6}+\lambda_{7}} \oplus 6 R_{\lambda_{3}+2 \lambda_{7}} \oplus 8 R_{\lambda_{1}+\lambda_{4}} \oplus 8 R_{\lambda_{2}+\lambda_{5}} \\
& \oplus 8 R_{2 \lambda_{1}+\lambda_{6}} \oplus R_{4 \lambda_{7}} \oplus 3 R_{\lambda_{6}+2 \lambda_{7}} \oplus 2 R_{3 \lambda_{1}} \oplus 9 R_{\lambda_{3}+\lambda_{6}} \oplus 4 R_{2 \lambda_{6}} \oplus 12 R_{\lambda_{1}+\lambda_{2}+\lambda_{7}} \oplus 6 R_{\lambda_{1}+\lambda_{3}} \\
& \oplus 3 R_{2 \lambda_{2}} \oplus 7 R_{\lambda_{5}+\lambda_{7}} \oplus 7 R_{\lambda_{4}} \oplus 6 R_{\lambda_{1}+2 \lambda_{7}} \oplus 7 R_{\lambda_{1}+\lambda_{6}} \oplus 5 R_{\lambda_{2}+\lambda_{7}} \oplus 2 R_{2 \lambda_{7}} \oplus 3 R_{2 \lambda_{1}} \oplus 3 R_{\lambda_{3}} \\
& \oplus 3 R_{\lambda_{6}} \oplus R_{\lambda_{1}} \oplus 1
\end{aligned}
$$




$$
\begin{aligned}
& R_{\lambda_{4}} \otimes R_{\lambda_{5}}=R_{\lambda_{4}+\lambda_{5}} \oplus R_{\lambda_{2}+\lambda_{3}+\lambda_{6}} \oplus R_{\lambda_{1}+\lambda_{5}+\lambda_{6}} \oplus R_{2 \lambda_{3}+\lambda_{7}} \oplus R_{\lambda_{1}+2 \lambda_{2}+\lambda_{7}} \oplus R_{\lambda_{1}+\lambda_{4}+\lambda_{7}} \oplus R_{\lambda_{2}+2 \lambda_{6}} \\
& \oplus \quad 2 R_{\lambda_{2}+\lambda_{5}+\lambda_{7}} \oplus 2 R_{\lambda_{1}+\lambda_{2}+\lambda_{3}} \oplus R_{2 \lambda_{1}+\lambda_{6}+\lambda_{7}} \oplus R_{3 \lambda_{2}} \oplus 2 R_{\lambda_{3}+\lambda_{6}+\lambda_{7}} \oplus 2 R_{\lambda_{2}+\lambda_{4}} \\
& \oplus \quad R_{2 \lambda_{1}+\lambda_{5}} \oplus 3 R_{\lambda_{3}+\lambda_{5}} \oplus 2 R_{\lambda_{1}+\lambda_{2}+2 \lambda_{7}} \oplus 5 R_{\lambda_{1}+\lambda_{2}+\lambda_{6}} \oplus R_{2 \lambda_{6}+\lambda_{7}} \oplus R_{\lambda_{5}+2 \lambda_{7}} \oplus R_{3 \lambda_{1}+\lambda_{7}} \\
& \oplus \quad 4 R_{\lambda_{1}+\lambda_{3}+\lambda_{7}} \oplus 3 R_{\lambda_{5}+\lambda_{6}} \oplus 3 R_{2 \lambda_{2}+\lambda_{7}} \oplus 5 R_{\lambda_{4}+\lambda_{7}} \oplus 3 R_{2 \lambda_{1}+\lambda_{2}} \oplus 5 R_{\lambda_{2}+\lambda_{3}} \oplus R_{\lambda_{1}+3 \lambda_{7}} \\
& \oplus 5 R_{\lambda_{1}+\lambda_{6}+\lambda_{7}} \oplus 5 R_{\lambda_{1}+\lambda_{5}} \oplus 3 R_{\lambda_{2}+2 \lambda_{7}} \oplus 5 R_{\lambda_{2}+\lambda_{6}} \oplus 4 R_{2 \lambda_{1}+\lambda_{7}} \oplus 5 R_{\lambda_{3}+\lambda_{7}} \oplus 4 R_{\lambda_{1}+\lambda_{2}} \oplus R_{3 \lambda_{7}} \\
& \oplus 3 R_{\lambda_{6}+\lambda_{7}} \oplus 3 R_{\lambda_{5}} \oplus 3 R_{\lambda_{1}+\lambda_{7}} \oplus R_{\lambda_{2}} \oplus R_{\lambda_{7}} \\
& R_{\lambda_{4}} \otimes R_{\lambda_{6}}=R_{\lambda_{4}+\lambda_{6}} \oplus R_{\lambda_{2}+\lambda_{3}+\lambda_{7}} \oplus R_{2 \lambda_{3}} \oplus R_{\lambda_{1}+\lambda_{5}+\lambda_{7}} \oplus R_{\lambda_{2}+\lambda_{6}+\lambda_{7}} \oplus R_{\lambda_{1}+2 \lambda_{2}} \oplus R_{\lambda_{1}+\lambda_{4}} \oplus R_{\lambda_{3}+2 \lambda_{7}} \\
& \oplus \quad 2 R_{\lambda_{2}+\lambda_{5}} \oplus R_{2 \lambda_{1}+\lambda_{6}} \oplus 2 R_{\lambda_{3}+\lambda_{6}} \oplus 3 R_{\lambda_{1}+\lambda_{2}+\lambda_{7}} \oplus R_{2 \lambda_{6}} \oplus 2 R_{\lambda_{5}+\lambda_{7}} \oplus R_{2 \lambda_{2}} \oplus R_{\lambda_{1}+2 \lambda_{7}} \\
& \oplus 2 R_{\lambda_{1}+\lambda_{3}} \oplus 3 R_{\lambda_{4}} \oplus 2 R_{\lambda_{1}+\lambda_{6}} \oplus 2 R_{\lambda_{2}+\lambda_{7}} \oplus R_{2 \lambda_{1}} \oplus R_{\lambda_{3}} \oplus R_{\lambda_{6}} \\
& R_{\lambda_{4}} \otimes R_{\lambda_{7}}=R_{\lambda_{4}+\lambda_{7}} \oplus R_{\lambda_{2}+\lambda_{3}} \oplus R_{\lambda_{1}+\lambda_{5}} \oplus R_{\lambda_{2}+\lambda_{6}} \oplus R_{\lambda_{3}+\lambda_{7}} \oplus R_{\lambda_{1}+\lambda_{2}} \oplus R_{\lambda_{5}} \\
& R_{\lambda_{5}} \otimes R_{\lambda_{5}}=R_{2 \lambda_{5}} \oplus R_{\lambda_{4}+\lambda_{6}} \oplus R_{\lambda_{2}+\lambda_{3}+\lambda_{7}} \oplus R_{\lambda_{1}+2 \lambda_{6}} \oplus R_{2 \lambda_{3}} \oplus R_{\lambda_{1}+\lambda_{5}+\lambda_{7}} \oplus 2 R_{\lambda_{2}+\lambda_{6}+\lambda_{7}} \\
& \oplus \quad R_{\lambda_{1}+2 \lambda_{2}} \oplus R_{2 \lambda_{1}+2 \lambda_{7}} \oplus R_{\lambda_{1}+\lambda_{4}} \oplus R_{\lambda_{3}+2 \lambda_{7}} \oplus 2 R_{\lambda_{2}+\lambda_{5}} \oplus R_{2 \lambda_{1}+\lambda_{6}} \oplus 3 R_{\lambda_{3}+\lambda_{6}} \oplus 4 R_{\lambda_{1}+\lambda_{2}+\lambda_{7}} \\
& \oplus \quad R_{\lambda_{6}+2 \lambda_{7}} \oplus R_{3 \lambda_{1}} \oplus 2 R_{2 \lambda_{6}} \oplus 3 R_{\lambda_{5}+\lambda_{7}} \oplus 2 R_{2 \lambda_{2}} \oplus 3 R_{\lambda_{1}+2 \lambda_{7}} \oplus 2 R_{\lambda_{1}+\lambda_{3}} \\
& \oplus 3 R_{\lambda_{4}} \oplus 4 R_{\lambda_{1}+\lambda_{6}} \oplus 3 R_{\lambda_{2}+\lambda_{7}} \oplus 2 R_{2 \lambda_{7}} \oplus 2 R_{2 \lambda_{1}} \oplus 2 R_{\lambda_{3}} \oplus 2 R_{\lambda_{6}} \oplus R_{\lambda_{1}} \oplus 1 \\
& R_{\lambda_{5}} \otimes R_{\lambda_{6}}=R_{\lambda_{5}+\lambda_{6}} \oplus R_{\lambda_{4}+\lambda_{7}} \oplus R_{\lambda_{1}+\lambda_{6}+\lambda_{7}} \oplus R_{\lambda_{2}+\lambda_{3}} \oplus R_{\lambda_{2}+2 \lambda_{7}} \oplus R_{\lambda_{1}+\lambda_{5}} \\
& \oplus \quad 2 R_{\lambda_{2}+\lambda_{6}} \oplus R_{2 \lambda_{1}+\lambda_{7}} \oplus 2 R_{\lambda_{3}+\lambda_{7}} \oplus 2 R_{\lambda_{1}+\lambda_{2}} \oplus 2 R_{\lambda_{6}+\lambda_{7}} \oplus 2 R_{\lambda_{5}} \oplus 2 R_{\lambda_{1}+\lambda_{7}} \oplus R_{\lambda_{2}} \oplus R_{\lambda_{7}} \\
& R_{\lambda_{5}} \otimes R_{\lambda_{7}}=R_{\lambda_{5}+\lambda_{7}} \oplus R_{\lambda_{4}} \oplus R_{\lambda_{6}} \oplus R_{\lambda_{3}} \oplus R_{\lambda_{1}+\lambda_{6}} \oplus R_{\lambda_{2}+\lambda_{7}} \\
& R_{\lambda_{6}} \otimes R_{\lambda_{6}}=R_{2 \lambda_{6}} \oplus R_{\lambda_{5}+\lambda_{7}} \oplus R_{\lambda_{1}} \oplus R_{\lambda_{3}} \oplus R_{\lambda_{4}} \oplus 2 R_{\lambda_{6}} \oplus R_{2 \lambda_{1}} \oplus R_{2 \lambda_{7}} \oplus R_{\lambda_{1}+\lambda_{6}} \oplus 2 R_{\lambda_{2}+\lambda_{7}} \\
& \oplus \quad R_{\lambda_{1}+2 \lambda_{7}} \oplus 1 \\
& R_{\lambda_{6}} \otimes R_{\lambda_{7}}=R_{\lambda_{6}+\lambda_{7}} \oplus R_{\lambda_{5}} \oplus R_{\lambda_{1}+\lambda_{7}} \oplus R_{\lambda_{2}} \oplus R_{\lambda_{7}} \\
& R_{\lambda_{7}} \otimes R_{\lambda_{7}}=R_{2 \lambda_{7}} \oplus R_{\lambda_{1}} \oplus R_{\lambda_{6}} \oplus 1
\end{aligned}
$$

We present also a list of second order characters:

$$
\begin{aligned}
\chi_{2000000} & =z_{1}^{2}-z_{3}-z_{6}-z_{1}-1 \\
\chi_{1100000} & =z_{1} z_{2}-z_{5}-z_{1} z_{7} \\
\chi_{1010000} & =z_{1} z_{3}-z_{4}-z_{1} z_{6}-z_{1}^{2}+z_{7}^{2}+z_{3} \\
\chi_{1001000} & =z_{1} z_{4}-z_{2} z_{5}+z_{6}^{2}-z_{5} z_{7}+z_{1} z_{6}-z_{2} z_{7}-z_{7}^{2}+z_{6}+z_{1} \\
\chi_{1000100} & =z_{1} z_{5}-z_{2} z_{6}+z_{1} z_{7}-z_{2} \\
\chi_{1000010} & =z_{1} z_{6}-z_{2} z_{7}-z_{7}^{2}+z_{6}+z_{1}+1 \\
\chi_{1000001} & =z_{1} z_{7}-z_{2}-z_{7} \\
\chi_{0200000} & =z_{2}^{2}-z_{4}-z_{1} z_{6}-z_{1}^{2}+z_{3}+z_{6}+z_{1} \\
\chi_{0110000} & =z_{2} z_{3}-z_{1} z_{5}-z_{1}^{2} z_{7}+z_{3} z_{7}+z_{6} z_{7}+z_{1} z_{7} \\
\chi_{0101000} & =z_{2} z_{4}-z_{3} z_{5}+z_{1} z_{6} z_{7}-z_{2} z_{7}^{2}-z_{1} z_{5}+z_{2} z_{6}-z_{6} z_{7}+z_{5}+z_{2} \\
\chi_{0100100} & =z_{2} z_{5}-z_{3} z_{6}-z_{6}^{2}+z_{5} z_{7}+z_{1} z_{7}^{2}-z_{1} z_{6}-z_{6}-z_{1} \\
\chi_{0100010} & =z_{2} z_{6}-z_{3} z_{7}-z_{6} z_{7}+z_{5}+z_{2} \\
\chi_{0100001} & =z_{2} z_{7}-z_{3}-z_{6}-z_{1} \\
\chi_{0020000} & =z_{3}^{2}-z_{1} z_{4}-z_{1}^{2} z_{6}+z_{3} z_{6}+z_{5} z_{7}-z_{1}^{3}+2 z_{1} z_{3}+z_{1} z_{7}^{2}-z_{4}+z_{3}+z_{1} \\
\chi_{0011000} & =z_{3} z_{4}-z_{1} z_{2} z_{5}+z_{4} z_{6}+z_{1} z_{6}^{2}+z_{1}^{2} z_{6}-z_{3} z_{6}-z_{6} z_{7}^{2}-z_{1} z_{2} z_{7}+z_{2}^{2}+z_{5} z_{7}-z_{4}-z_{1} z_{6}+z_{7}^{2} \\
& +z_{3}-1
\end{aligned}
$$




$$
\begin{aligned}
\chi_{0010100} & =z_{3} z_{5}-z_{1} z_{2} z_{6}+z_{4} z_{7}+z_{1} z_{6} z_{7}+z_{2} z_{7}^{2}-z_{7}^{3}+z_{1}^{2} z_{7}-z_{2} z_{6}-z_{3} z_{7}-z_{1} z_{2}+z_{6} z_{7}-z_{5}-z_{1} z_{7} \\
& -z_{2}+z_{7} \\
\chi_{0010010} & =z_{3} z_{6}-z_{1} z_{2} z_{7}+z_{4}+z_{1} z_{6}+z_{2} z_{7}+z_{1}^{2}-z_{3}-z_{1} \\
\chi_{0010001} & =z_{3} z_{7}-z_{1} z_{2}+z_{7} \\
\chi_{0002000} & =z_{4}^{2}-z_{2} z_{3} z_{5}+z_{1} z_{4} z_{6}+z_{3} z_{6}^{2}+z_{1}^{2} z_{5} z_{7}-2 z_{3} z_{5} z_{7}-2 z_{4} z_{7}^{2}-z_{1} z_{6} z_{7}^{2}-z_{5}^{2}+2 z_{4} z_{6}+z_{1} z_{6}^{2} \\
& -z_{1} z_{5} z_{7}+z_{2} z_{5}+z_{7}^{4}-2 z_{6} z_{7}^{2}+z_{6}^{2}+2 z_{4}+z_{1} z_{6}-2 z_{7}^{2}+2 z_{6}+1 \\
\chi_{0001100} & =z_{4} z_{5}-z_{2} z_{3} z_{6}+z_{1} z_{4} z_{7}+z_{1}^{2} z_{6} z_{7}-z_{3} z_{5}-z_{5} z_{6}-z_{1} z_{7}^{3}-z_{4} z_{7}-z_{1} z_{5}+z_{7}^{3}-z_{6} z_{7}+z_{1} z_{7}-z_{7} \\
\chi_{0001010} & =z_{4} z_{6}-z_{2} z_{3} z_{7}+z_{1}^{2} z_{7}^{2}+z_{1} z_{4}-z_{3} z_{7}^{2}+z_{3} z_{6}-z_{5} z_{7}-2 z_{1} z_{7}^{2}+z_{4}+z_{1} z_{6}-z_{7}^{2}+z_{6}+z_{1}+1 \\
\chi_{0001001} & =z_{4} z_{7}-z_{2} z_{3}+z_{1}^{2} z_{7}-z_{3} z_{7}-z_{5}-z_{1} z_{7}-z_{7} \\
\chi_{0000200} & =z_{5}^{2}-z_{4} z_{6}-z_{1} z_{6}^{2}+z_{1} z_{5} z_{7}+z_{3} z_{7}^{2}-z_{3} z_{6}+z_{6} z_{7}^{2}-z_{6}^{2}-z_{4}-z_{1} z_{6}+z_{7}^{2}-z_{3}-2 z_{6}-1 \\
\chi_{0000110} & =z_{5} z_{6}-z_{4} z_{7}-z_{1} z_{6} z_{7}+z_{1} z_{5}+z_{7}^{3}+z_{3} z_{7}-z_{6} z_{7}+z_{5}-z_{7} \\
\chi_{0000101} & =z_{5} z_{7}-z_{4}-z_{1} z_{6}+z_{7}^{2}-z_{6}-1 \\
\chi_{0000020} & =z_{6}^{2}-z_{5} z_{7}-z_{1} z_{7}^{2}+z_{1} z_{6}+z_{3}+z_{6}+z_{1} \\
\chi_{0000011} & =z_{6} z_{7}-z_{5}-z_{1} z_{7} \\
\chi_{0000002} & =z_{7}^{2}-z_{6}-z_{1}-1
\end{aligned}
$$

\section{The Calogero-Sutherland Hamiltonian $\Delta^{1}$ in $E_{7}$. Some applications}

The coefficients $b_{j}(z)$ in the expression of $\Delta^{1}$ are easily obtained from (15) and (2):

$$
\begin{aligned}
& b_{1}(z)=72 z_{1} ; \quad b_{2}(z)=105 z_{2} ; \quad b_{3}(z)=144 z_{1} ; \quad b_{4}(z)=216 z_{4} ; \\
& b_{5}(z)=165 z_{5} ; \quad b_{6}(z)=112 z_{5} ; \quad b_{7}(z)=57 z_{7} .
\end{aligned}
$$

After having computed in Section 4 the necessary series and characters, we can now follow the lines indicated in Section 3 to obtain the Hamiltonian operator in the limit $\kappa=1$. The result for the coefficients $a_{j k}(z)$ in $(14)$ for $\kappa=1$ is

$$
\begin{aligned}
a_{11}(z) & =4\left(-19-10 z_{1}+z_{1}^{2}-z_{3}-5 z_{6}\right) \\
a_{12}(z) & =2\left(-7 z_{2}+2 z_{1} z_{2}-5 z_{5}-19 z_{7}-13 z_{1} z_{7}\right) \\
a_{13}(z) & =2\left(10-14 z_{1}-19 z_{1}^{2}+13 z_{3}+3 z_{1} z_{3}-3 z_{4}-4 z_{6}-9 z_{1} z_{6}-6 z_{2} z_{7}+9 z_{7}^{2}\right) \\
a_{14}(z) & =2\left(-10-2 z_{1}+18 z_{1}^{2}-7 z_{2}^{2}-24 z_{3}-6 z_{1} z_{3}+14 z_{4}+4 z_{1} z_{4}-4 z_{2} z_{5}+8 z_{6}+12 z_{1} z_{6}\right. \\
& \left.-4 z_{3} z_{6}+4 z_{6}^{2}-14 z_{2} z_{7}-5 z_{1} z_{2} z_{7}-9 z_{5} z_{7}-9 z_{7}^{2}+9 z_{1} z_{7}^{2}\right) \\
a_{15}(z) & =2\left(-12 z_{2}-6 z_{1} z_{2}+8 z_{5}+3 z_{1} z_{5}-5 z_{2} z_{6}+19 z_{7}+5 z_{1} z_{7}-5 z_{3} z_{7}-13 z_{6} z_{7}\right) \\
a_{16}(z) & =4\left(9-3 z_{1}-3 z_{3}+2 z_{6}+z_{1} z_{6}-3 z_{2} z_{7}-9 z_{7}^{2}\right) \\
a_{17}(z) & =2\left(-7 z_{2}-19 z_{7}+z_{1} z_{7}\right) \\
a_{22}(z) & =-40+24 z_{1}-36 z_{1}^{2}+7 z_{2}^{2}+24 z_{3}-4 z_{4}+44 z_{6}-16 z_{1} z_{6}-12 z_{2} z_{7}-36 z_{7}^{2} \\
a_{23}(z) & =2\left(9 z_{2}-14 z_{1} z_{2}+4 z_{2} z_{3}+16 z_{5}-4 z_{1} z_{5}-5 z_{2} z_{6}+4 z_{1} z_{7}-12 z_{1}^{2} z_{7}+7 z_{3} z_{7}-z_{6} z_{7}\right) \\
a_{24}(z) & =2\left(-7 z_{2}-4 z_{1} z_{2}-6 z_{1}^{2} z_{2}-z_{2} z_{3}+6 z_{2} z_{4}+20 z_{5}-6 z_{1} z_{5}-3 z_{3} z_{5}+14 z_{2} z_{6}-4 z_{1} z_{2} z_{6}\right. \\
& -5 z_{5} z_{6}-10 z_{1} z_{7}+22 z_{1}^{2} z_{7}-6 z_{2}^{2} z_{7}-12 z_{3} z_{7}-5 z_{1} z_{3} z_{7}+12 z_{4} z_{7} \\
& \left.-10 z_{6} z_{7}+13 z_{1} z_{6} z_{7}-9 z_{2} z_{7}^{2}\right) \\
a_{25}(z) & =40-24 z_{1}+12 z_{1}^{2}-14 z_{2}^{2}+12 z_{3}-12 z_{1} z_{3}+28 z_{4}+9 z_{2} z_{5}+16 z_{6}-12 z_{1} z_{6}
\end{aligned}
$$


$-8 z_{3} z_{6}-24 z_{6}^{2}+12 z_{2} z_{7}-10 z_{1} z_{2} z_{7}+14 z_{5} z_{7}-2 z_{7}^{2}-2 z_{1} z_{7}^{2}$

$$
\begin{aligned}
a_{26}(z) & =2\left(17 z_{2}-6 z_{1} z_{2}+8 z_{5}+3 z_{2} z_{6}-24 z_{1} z_{7}-5 z_{3} z_{7}-13 z_{6} z_{7}\right) \\
a_{27}(z) & =-48 z_{1}-12 z_{3}-28 z_{6}+3 z_{2} z_{7} \\
a_{33}(z) & =4\left(-20+16 z_{1}-5 z_{1}^{2}-9 z_{1}^{3}+8 z_{3}+12 z_{1} z_{3}+3 z_{3}^{2}-7 z_{4}-z_{1} z_{4}-2 z_{2} z_{5}-9 z_{6}\right. \\
& \left.+-3 z_{1} z_{6}-4 z_{1}^{2} z_{6}+2 z_{3} z_{6}-3 z_{6}^{2}+6 z_{2} z_{7}-6 z_{1} z_{2} z_{7}+8 z_{5} z_{7}+z_{7}^{2}+7 z_{1} z_{7}^{2}\right) \\
a_{34}(z) & =2\left(-20-12 z_{1}-6 z_{1}^{2}+6 z_{1}^{3}+9 z_{2}^{2}-7 z_{1} z_{2}^{2}+20 z_{3}+6 z_{1} z_{3}-6 z_{1}^{2} z_{3}+6 z_{3}^{2}-26 z_{4}+6 z_{1} z_{4}\right. \\
& +8 z_{3} z_{4}+2 z_{2} z_{5}-3 z_{1} z_{2} z_{5}-5 z_{5}^{2}-2 z_{6}-32 z_{1} z_{6}+20 z_{1}^{2} z_{6}-5 z_{2}^{2} z_{6}-24 z_{3} z_{6}-4 z_{1} z_{3} z_{6} \\
& +10 z_{4} z_{6}+4 z_{6}^{2}+4 z_{1} z_{6}^{2}-3 z_{2} z_{7}-2 z_{1} z_{2} z_{7}-5 z_{1}^{2} z_{2} z_{7}-z_{2} z_{3} z_{7}+15 z_{5} z_{7}+4 z_{2} z_{6} z_{7} \\
& \left.+20 z_{7}^{2}-5 z_{1} z_{7}^{2}+5 z_{1}^{2} z_{7}^{2}+9 z_{3} z_{7}^{2}-9 z_{6} z_{7}^{2}\right)
\end{aligned}
$$

$a_{35}(z)=2\left(-z_{2}+z_{1} z_{2}-6 z_{1}^{2} z_{2}-z_{2} z_{3}-22 z_{5}+15 z_{1} z_{5}+6 z_{3} z_{5}-15 z_{2} z_{6}-4 z_{1} z_{2} z_{6}-5 z_{5} z_{6}\right.$

$+18 z_{7}-5 z_{1} z_{7}+11 z_{1}^{2} z_{7}-6 z_{2}^{2} z_{7}-6 z_{3} z_{7}-5 z_{1} z_{3} z_{7}+12 z_{4} z_{7}+45 z_{6} z_{7}-8 z_{1} z_{6} z_{7}$

$\left.+12 z_{2} z_{7}^{2}-18 z_{7}^{3}\right)$

$a_{36}(z)=2\left(20+6 z_{1}^{2}-7 z_{2}^{2}-18 z_{3}-6 z_{1} z_{3}+14 z_{4}+20 z_{6}+6 z_{1} z_{6}+4 z_{3} z_{6}+6 z_{2} z_{7}-5 z_{1} z_{2} z_{7}\right.$

$\left.-5 z_{5} z_{7}-z_{7}^{2}-13 z_{1} z_{7}^{2}\right)$

$a_{37}(z)=2\left(-7 z_{2}-6 z_{1} z_{2}-5 z_{5}+19 z_{7}-13 z_{1} z_{7}+2 z_{3} z_{7}\right)$

$a_{44}(z)=4\left(-10-16 z_{1}+8 z_{1}^{2}-6 z_{1}^{4}-4 z_{2}^{2}-16 z_{3}+24 z_{1}^{2} z_{3}-4 z_{2}^{2} z_{3}-12 z_{3}^{2}+8 z_{4}-16 z_{1} z_{4}-4 z_{1}^{2} z_{4}\right.$

$+8 z_{3} z_{4}+6 z_{4}^{2}+11 z_{2} z_{5}+2 z_{1} z_{2} z_{5}-z_{2} z_{3} z_{5}-12 z_{5}^{2}-2 z_{1} z_{5}^{2}+4 z_{6}-4 z_{1} z_{6}-14 z_{1}^{2} z_{6}+4 z_{1}^{3} z_{6}$

$+3 z_{2}^{2} z_{6}-2 z_{1} z_{2}^{2} z_{6}-4 z_{3} z_{6}-8 z_{1} z_{3} z_{6}-2 z_{3}^{2} z_{6}+6 z_{4} z_{6}+4 z_{1} z_{4} z_{6}-3 z_{2} z_{5} z_{6}-2 z_{6}^{2}+4 z_{1} z_{6}^{2}$

$+4 z_{3} z_{6}^{2}-2 z_{6}^{3}+z_{2} z_{7}+z_{1} z_{2} z_{7}+9 z_{1}^{2} z_{2} z_{7}-3 z_{2}^{3} z_{7}-8 z_{2} z_{3} z_{7}-3 z_{1} z_{2} z_{3} z_{7}+9 z_{2} z_{4} z_{7}-21 z_{5} z_{7}$

$+6 z_{1} z_{5} z_{7}+4 z_{1}^{2} z_{5} z_{7}-9 z_{3} z_{5} z_{7}-6 z_{2} z_{6} z_{7}+7 z_{1} z_{2} z_{6} z_{7}+9 z_{5} z_{6} z_{7}-9 z_{7}^{2}+17 z_{1} z_{7}^{2}-3 z_{1}^{2} z_{7}^{2}$

$\left.-2 z_{1}^{3} z_{7}^{2}+9 z_{1} z_{3} z_{7}^{2}-9 z_{4} z_{7}^{2}+9 z_{6} z_{7}^{2}-9 z_{1} z_{6} z_{7}^{2}\right)$

$a_{45}(z)=2\left(9 z_{2}-7 z_{1} z_{2}+13 z_{1}^{2} z_{2}-7 z_{2}^{3}-12 z_{2} z_{3}-7 z_{1} z_{2} z_{3}+21 z_{2} z_{4}-28 z_{5}-7 z_{1} z_{5}+7 z_{1}^{2} z_{5}\right.$

$-13 z_{3} z_{5}+9 z_{4} z_{5}-3 z_{2} z_{6}+7 z_{1} z_{2} z_{6}-3 z_{2} z_{3} z_{6}-19 z_{5} z_{6}-4 z_{1} z_{5} z_{6}-5 z_{2} z_{6}^{2}+21 z_{1} z_{7}$

$-24 z_{1}^{2} z_{7}+6 z_{1}^{3} z_{7}+7 z_{2}^{2} z_{7}-5 z_{1} z_{2}^{2} z_{7}+14 z_{3} z_{7}-z_{1} z_{3} z_{7}-5 z_{3}^{2} z_{7}-19 z_{4} z_{7}+10 z_{1} z_{4} z_{7}$

$\left.-z_{2} z_{5} z_{7}+10 z_{1} z_{6} z_{7}+4 z_{1}^{2} z_{6} z_{7}+5 z_{6}^{2} z_{7}-2 z_{2} z_{7}^{2}+4 z_{1} z_{2} z_{7}^{2}+9 z_{5} z_{7}^{2}-9 z_{1} z_{7}^{3}\right)$

$a_{46}(z)=2\left(20+12 z_{1}-18 z_{1}^{2}+12 z_{1}^{3}+7 z_{2}^{2}-6 z_{1} z_{2}^{2}+12 z_{3}-24 z_{1} z_{3}-6 z_{3}^{2}+2 z_{4}\right.$

$+12 z_{1} z_{4}-z_{2} z_{5}+2 z_{6}+8 z_{1} z_{6}+2 z_{1}^{2} z_{6}+8 z_{3} z_{6}+6 z_{4} z_{6}-4 z_{6}^{2}-6 z_{2} z_{7}-z_{1} z_{2} z_{7}$

$\left.-4 z_{2} z_{3} z_{7}-10 z_{5} z_{7}-4 z_{1} z_{5} z_{7}-5 z_{2} z_{6} z_{7}-20 z_{7}^{2}+2 z_{1} z_{7}^{2}+4 z_{1}^{2} z_{7}^{2}-9 z_{3} z_{7}^{2}+9 z_{6} z_{7}^{2}\right)$

$a_{47}(z)=2\left(-5 z_{2}-6 z_{1} z_{2}-5 z_{2} z_{3}-19 z_{5}-4 z_{1} z_{5}-5 z_{2} z_{6}-19 z_{7}+11 z_{1} z_{7}+5 z_{1}^{2} z_{7}-10 z_{3} z_{7}\right.$

$\left.+3 z_{4} z_{7}+9 z_{6} z_{7}\right)$

$a_{55}(z)=-60+48 z_{1}-12 z_{1}^{2}-12 z_{1} z_{2}^{2}-24 z_{3}+24 z_{1} z_{3}-12 z_{3}^{2}-48 z_{4}+24 z_{1} z_{4}+12 z_{2} z_{5}$

$+15 z_{5}^{2}-52 z_{6}+24 z_{1}^{2} z_{6}-48 z_{3} z_{6}-4 z_{4} z_{6}-48 z_{6}^{2}-16 z_{1} z_{6}^{2}+40 z_{2} z_{7}+8 z_{1} z_{2} z_{7}$

$-8 z_{2} z_{3} z_{7}+20 z_{5} z_{7}+8 z_{1} z_{5} z_{7}-24 z_{2} z_{6} z_{7}-16 z_{7}^{2}+4 z_{1} z_{7}^{2}-12 z_{1}^{2} z_{7}^{2}+32 z_{3} z_{7}^{2}+28 z_{6} z_{7}^{2}$

$a_{56}(z)=2\left(7 z_{2}-z_{1} z_{2}-5 z_{2} z_{3}+8 z_{5}+5 z_{1} z_{5}-7 z_{2} z_{6}+5 z_{5} z_{6}-28 z_{7}+19 z_{1} z_{7}-6 z_{1}^{2} z_{7}\right.$

$\left.+11 z_{3} z_{7}-3 z_{4} z_{7}-17 z_{6} z_{7}-9 z_{1} z_{6} z_{7}-6 z_{2} z_{7}^{2}+9 z_{7}^{3}\right)$

$a_{57}(z)=-20+12 z_{1}-12 z_{3}-8 z_{4}-48 z_{6}-20 z_{1} z_{6}-12 z_{2} z_{7}+5 z_{5} z_{7}+20 z_{7}^{2}$

$a_{66}(z)=4\left(-14+12 z_{1}-6 z_{1}^{2}+12 z_{3}-2 z_{4}+2 z_{6}+2 z_{6}^{2}-7 z_{2} z_{7}-z_{5} z_{7}-5 z_{7}^{2}-5 z_{1} z_{7}^{2}\right)$

$a_{67}(z)=2\left(-7 z_{2}-3 z_{5}-19 z_{7}-11 z_{1} z_{7}+2 z_{6} z_{7}\right)$

$a_{77}(z)=-60-24 z_{1}-4 z_{6}+3 z_{7}^{2}$ 
With the explicit expression of $\Delta^{1}$ at our disposal, we can now try to use the Schrödinger equation as an efficient mean to compute particular characters of $E_{7}$. Given that all these characters are polynomials in the $z$-variables, the Schrödinger equation can be solved by applying a systematic procedure, which is suitable to be implemented in a computer program able to carry out symbolic calculations. We propose two alternative methods to find the Schrödinger eigenfunctions:

1. Given a weight $\nu=\sum_{i=1}^{7} n_{i} \lambda_{i} \in P^{+}$, let us denote $z^{\mathbf{n}}$ (or $z^{\nu}$ ) the monomial $z^{\mathbf{n}}=\prod_{i=1}^{7} z_{i}^{n_{i}}$; thus $z_{i}=z^{\lambda_{i}}$. The operator $\Delta^{1}$ acting on $z^{\mathbf{n}}$ gives

$$
\Delta^{1} z^{\mathbf{n}}=\sum_{\beta \in \Lambda} S_{\beta, \mathbf{n}} z^{\mathbf{n}-\beta}=\varepsilon_{\mathbf{m}}(1) z^{\mathbf{m}}+\sum_{0 \neq \beta \in \Lambda} S_{\beta, \mathbf{n}} z^{\mathbf{n}-\beta},
$$

where $\Lambda$ only includes integral linear combinations of the simple roots with non-negative coefficients and, of course, in the exponent of (20) we express $\beta$ in the basis of fundamental weights. The eigenfunctions $\chi_{\mathbf{m}}$ can be written as

$$
\chi_{\mathbf{m}}=\sum_{\lambda \in Q_{\mathbf{m}}^{+}} C_{\lambda} z^{\mathbf{m}-\lambda}=z^{\mathbf{m}}+\sum_{0 \neq \lambda \in Q_{\mathbf{m}}^{+}} C_{\lambda} z^{\mathbf{m}-\lambda},
$$

where again the $\lambda$ in $Q_{\mathbf{m}}^{+}$are integral linear combinations of the simple roots with non-negative coefficients such that they do not give rise to negative powers of the $z$ 's. By substituting in the Schrödinger equation $\Delta^{1} \chi_{\mathbf{m}}=\varepsilon_{\mathbf{m}}(1) \chi_{\mathbf{m}}$ we find the iterative formula

$$
C_{\lambda}=\frac{1}{\varepsilon_{\mathbf{m}}(1)-\varepsilon_{\mathbf{m}-\lambda}(1)} \sum_{0 \neq \beta \in \Lambda} S_{\beta, \mathbf{m}-(\lambda-\beta)} C_{\lambda-\beta} .
$$

To use this formula in practice, one should take into account the heights of the $\lambda^{\prime} s$ involved, because each coefficient $C_{\lambda}$ can depend only on some of the $C_{\nu}$ such that $\operatorname{ht}(\nu)<\operatorname{ht}(\lambda)$.

2. The Clebsch-Gordan series for the product $\prod_{i=1}^{7} z_{i}^{m_{i}}$ reads

$$
z_{1}^{m_{1}} z_{2}^{m_{2}} z_{3}^{m_{3}} z_{4}^{m_{4}} z_{5}^{m_{5}} z_{6}^{m_{6}} z_{7}^{m_{7}}=\chi_{\mathbf{m}}+\sum_{\beta \in Q_{\mathbf{m}}} D_{\beta} \boldsymbol{\chi}_{\mathbf{m}-\beta}
$$

Here it is not difficult, in each particular case, to elaborate a list with all the elements in $Q_{\mathbf{m}}$ (i.e., the integral dominant weights appearing in the series). Furthermore, the operator $\Delta^{1}-\varepsilon_{\mathbf{n}}(1)$ annihilates the character $\chi_{\mathbf{n}}$. Having this into account, we can make use of the simple-looking formula

$$
\chi_{\mathbf{m}}=\left\{\prod_{\beta \in Q_{\mathbf{m}}}\left(\Delta^{1}-\varepsilon_{\mathbf{m}-\beta}(1)\right)\right\} z^{\mathbf{m}}
$$

to obtain the eigenfunctions.

Through any of these methods, it is possible to compute the characters rather quickly. As an illustration, we offer a list of the third order characters in the Appendix A.

Once we have a method for the computation of the characters, we can extend it to produce an algorithm for calculating the Clebsch-Gordan series. Suppose that we want to obtain the series for $\chi_{\mathbf{m}} \cdot \chi_{\mathbf{n}}$. We list the possible dominant weights entering in the series arranged by heights

$$
\chi_{\mathbf{m}} \cdot \chi_{\mathbf{n}}=\chi_{\mathbf{m}+\mathbf{n}}+N_{\mu_{1}} \chi_{\mu_{1}}+N_{\mu_{2}} \chi_{\mu_{2}}+\ldots
$$


The multiplicity $N_{\mu_{1}}$ is simply the difference between the coefficients of $z^{\mu_{1}}$ in $\chi_{\mathbf{m}} \cdot \chi_{\mathbf{n}}$ and in $\chi_{\mathbf{m}+\mathbf{n}}$. Then, $N_{\mu_{2}}$ is the difference between the coefficient of $z^{\mu_{2}}$ in $\chi_{\mathbf{m}} \cdot \chi_{\mathbf{n}}$ and the sum of the corresponding coefficients in $\chi_{\mathbf{m}+\mathbf{n}}$ and $\chi_{\mu_{1}}$, and so on. As an example, we present in Appendix B a list with all the cubic Clebsch-Gordan series.

The approach we are describing is also useful to find the general structure of the series for products of some specific types. Let us consider, for instance, series of the type $z_{7} \chi_{n \lambda_{7}}$ with arbitrary integer $n>0$. The weights of the representation $R_{\lambda_{7}}$ are given by the linear combinations $\pm\left(v_{i}+v_{j}\right), i \neq j[22]$. If we expand these weights in the basis of fundamental weights, we see that there are only four whose coefficients for all $\lambda_{i}$ with $i \neq 7$ are non-negative: $\lambda_{7}, \lambda_{6}-\lambda_{7}, \lambda_{1}-\lambda_{7}$ and $-\lambda_{7}$. Hence, the form of the series should be

$$
z_{7} \chi_{0,0,0,0,0,0, n}=\chi_{0,0,0,0,0,0, n+1}+a \chi_{0,0,0,0,0,1, n-1}+b \chi_{1,0,0,0,0,0, n-1}+c \chi_{0,0,0,0,0,0, n-1},
$$

where we have to fix $a, b$ and $c$. Now, by solving the Schrödinger equation by means of the first of the two methods described above, one finds

$$
\begin{aligned}
\chi_{0,0,0,0,0,0, n} & =z_{7}^{n}+(1-n) z_{6} z_{7}^{n-2}-z_{1} z_{7}^{n-2}+\ldots \\
\chi_{0,0,0,0,0,1, n-1} & =z_{6} z_{7}^{n-1}-z_{1} z_{7}^{n-1}+\ldots
\end{aligned}
$$

If we substitute this in (21), we can solve for $a$ and $b$, obtaining $a=b=1$. We can now fix $c$ by adjusting dimensions in (21). This gives $c=1$.

We list below the series of the form $z_{7} \chi_{n \lambda_{k}}$ obtained through the same procedure:

$$
\begin{aligned}
z_{7} \chi_{n, 0,0,0,0,0,0} & =\chi_{n, 0,0,0,0,0,1}+\chi_{n-1,1,0,0,0,0,0}+\chi_{n-1,0,0,0,0,0,1} \\
z_{7} \chi_{0, n, 0,0,0,0,0} & =\chi_{0, n, 0,0,0,0,1}+\chi_{0, n-1,1,0,0,0,0}+\chi_{0, n-1,0,0,0,1,0}+\chi_{1, n-1,0,0,0,0,0} \\
z_{7} \chi_{0,0, n, 0,0,0,0} & =\chi_{0,0, n, 0,0,0,1}+\chi_{1,1, n-1,0,0,0,0}+\chi_{0,0, n-1,0,1,0,0}+\chi_{1,0, n-1,0,0,0,1}+\chi_{0,1, n-1,0,0,0,0} \\
z_{7} \chi_{0,0,0, n, 0,0,0} & =\chi_{0,0,0, n, 0,0,1}+\chi_{0,1,1, n-1,0,0,0}+\chi_{1,0,0, n-1,1,0,0}+\chi_{0,1,0, n-1,0,1,0}+\chi_{0,0,1, n-1,0,0,1} \\
& +\chi_{1,1,0, n-1,0,0,0}+\chi_{0,0,0, n-1,1,0,0} \\
z_{7} \chi_{0,0,0,0, n, 0,0} & =\chi_{0,0,0,0, n, 0,1}+\chi_{0,0,0,1, n-1,0,0}+\chi_{1,0,0,0, n-1,1,0}+\chi_{0,1,0,0, n-1,0,1}+\chi_{0,0,1,0, n-1,0,0} \\
& +\chi_{0,0,0,0, n-1,1,0} \\
z_{7} \chi_{0,0,0,0,0, n, 0} & =\chi_{0,0,0,0,0, n, 1}+\chi_{0,0,0,0,1, n-1,0}+\chi_{1,0,0,0,0, n-1,1}+\chi_{0,1,0,0,0, n-1,0}+\chi_{0,0,0,0,0, n-1,1}
\end{aligned}
$$

\section{Conclusions}

In this paper we have shown how the Calogero-Sutherland Hamiltonian for the Lie algebra $E_{7}$ can be used to compute both Clebsch-Gordan series and characters of that algebra. The treatment we have presented can be applied to the cases of other simple algebras. It can be also extended to deal with the system of orthogonal polynomials based on $E_{7}$ for general values of the parameter $\kappa$. The way in which this should be done is the subject of a research now in progress and will be published elsewhere.

\section{Acknowledgements}

This work has been partially supported by the Spanish Ministerio de Educación y Ciencia under grants BFM2003-02532 (J.F.N.), BFM2003-00936 / FISI (W.G.F. and A.M.P.), and the sabbatical SAB-20030256 (A.M.P.). 


\section{Appendix A: List of the characters of $E_{7}$ of third order.}

$$
\begin{aligned}
& \chi_{3000000}=z_{1}^{3}-2 z_{1} z_{3}+z_{4}-z_{1} z_{6}-z_{1}^{2}+z_{2} z_{7}-z_{3}-2 z_{1} \\
& \chi_{2100000}=z_{1}^{2} z_{2}-z_{2} z_{3}-z_{1} z_{5}-z_{1}^{2} z_{7}+z_{6} z_{7}-z_{5}-z_{2} \\
& \chi_{1200000}=z_{1} z_{2}^{2}-z_{1} z_{4}-z_{2} z_{5}-z_{1}^{2} z_{6}+z_{3} z_{6}+z_{6}^{2}-z_{1} z_{2} z_{7}-z_{1}^{3}+2 z_{1} z_{3}-z_{4}+2 z_{1} z_{6}+z_{1}^{2}-z_{2} z_{7} \\
& +z_{3}+z_{6}+z_{1} \\
& \chi_{0300000}=z_{2}^{3}-2 z_{2} z_{4}+z_{3} z_{5}-2 z_{1} z_{2} z_{6}+z_{5} z_{6}+z_{1} z_{3} z_{7}-z_{4} z_{7}-2 z_{1}^{2} z_{2}+2 z_{2} z_{3}+z_{1} z_{5}+z_{2} z_{6} \\
& +z_{3} z_{7}+z_{1} z_{2}+z_{6} z_{7}+z_{1} z_{7} \\
& \chi_{2010000}=z_{1}^{2} z_{3}-z_{3}^{2}-z_{1} z_{4}-z_{1}^{2} z_{6}+z_{2} z_{5}-z_{3} z_{6}+z_{1} z_{2} z_{7}-z_{1}^{3}+z_{1} z_{7}^{2}-z_{2} z_{7}-z_{7}^{2}+z_{6}+z_{1}+1 \\
& \chi_{1110000}=z_{1} z_{2} z_{3}-z_{2} z_{4}-z_{1}^{2} z_{5}+z_{5} z_{6}-z_{1}^{3} z_{7}+z_{1} z_{3} z_{7}-z_{4} z_{7}+z_{1} z_{6} z_{7}+z_{1} z_{5}-z_{2} z_{6}+z_{1}^{2} z_{7}-z_{6} z_{7} \\
& +z_{5}+z_{1} z_{7} \\
& \chi_{0210000}=z_{2}^{2} z_{3}-z_{3} z_{4}-z_{1} z_{2} z_{5}+z_{5}^{2}-z_{4} z_{6}-z_{1}^{2} z_{2} z_{7}+z_{2} z_{3} z_{7}+z_{1} z_{5} z_{7}-z_{1} z_{4}+z_{2} z_{5}+z_{1} z_{2} z_{7}-z_{6}^{2} \\
& +z_{5} z_{7}+z_{1} z_{7}^{2}-z_{1} z_{6}+z_{2} z_{7}-z_{3}-z_{6}-z_{1} \\
& \chi_{1020000}=z_{1} z_{3}^{2}-z_{1}^{2} z_{4}-z_{3} z_{4}+z_{1} z_{2} z_{5}-z_{1}^{3} z_{6}+z_{1}^{2} z_{2} z_{7}-z_{1}^{4}-z_{2} z_{3} z_{7}+z_{1}^{2} z_{3}+z_{1} z_{5} z_{7}+z_{3}^{2}+z_{1}^{2} z_{7}^{2} \\
& -z_{2} z_{6} z_{7}-z_{1} z_{4}+z_{3} z_{6}-z_{1} z_{2} z_{7}+2 z_{1} z_{3}-z_{5} z_{7}-z_{1} z_{7}^{2}+z_{4}+2 z_{1} z_{6}+2 z_{1}^{2} \\
& \chi_{0120000}=z_{2} z_{3}^{2}-z_{1} z_{2} z_{4}-z_{1} z_{3} z_{5}+z_{4} z_{5}-z_{1}^{2} z_{3} z_{7}+z_{1} z_{5} z_{6}+z_{3}^{2} z_{7}-z_{2} z_{6}^{2}+z_{2} z_{5} z_{7}+z_{3} z_{6} z_{7}+z_{1}^{2} z_{5} \\
& -z_{3} z_{5}-z_{1} z_{2} z_{6}-z_{5} z_{6}+z_{1} z_{3} z_{7}+z_{2}^{2} z_{7}+z_{2} z_{7}^{2}-z_{1} z_{5}-2 z_{2} z_{6}+z_{3} z_{7}-z_{1} z_{2}+z_{6} z_{7}-z_{5}-z_{2} \\
& \chi_{0030000}=z_{3}^{3}-2 z_{1} z_{3} z_{4}+z_{4}^{2}+z_{1}^{2} z_{2} z_{5}-z_{2} z_{3} z_{5}-2 z_{1}^{2} z_{3} z_{6}+2 z_{3}^{2} z_{6}+z_{1} z_{4} z_{6}-z_{2} z_{5} z_{6}+z_{3} z_{6}^{2} \\
& +z_{1}^{3} z_{2} z_{7}-2 z_{1}^{3} z_{3}-2 z_{1} z_{2} z_{3} z_{7}+z_{2} z_{4} z_{7}+z_{3} z_{5} z_{7}-z_{1} z_{2} z_{6} z_{7}+4 z_{1} z_{3}^{2}+z_{1}^{2} z_{4}-3 z_{3} z_{4} \\
& +z_{1} z_{3} z_{7}^{2}-z_{1} z_{2} z_{5}+z_{5} z_{6} z_{7}-z_{5}^{2}-z_{4} z_{7}^{2}+2 z_{1} z_{3} z_{6}-z_{1}^{2} z_{2} z_{7}+z_{2}^{2} z_{6}-z_{4} z_{6}-z_{1} z_{5} z_{7}-z_{1}^{2} z_{7}^{2} \\
& +z_{1}^{2} z_{3}+2 z_{3}^{2}+z_{3} z_{7}^{2}-z_{1} z_{4}-z_{2} z_{5}+2 z_{3} z_{6}+z_{6} z_{7}^{2}-z_{1} z_{2} z_{7}+2 z_{1} z_{3}+z_{1} z_{7}^{2}+z_{4} \\
& +z_{1} z_{6}+z_{1}^{2}-z_{3}-z_{6}-z_{1} \\
& \chi_{2001000}=z_{1}^{2} z_{4}-z_{3} z_{4}-z_{1} z_{2} z_{5}+z_{5}^{2}+z_{2}^{2} z_{6}-2 z_{4} z_{6}-z_{2} z_{6} z_{7}+z_{3} z_{7}^{2}-z_{1} z_{4}+z_{2} z_{5}-2 z_{1} z_{2} z_{7} \\
& +z_{5} z_{7}+z_{2}^{2}-z_{4}+z_{1} z_{6}+z_{1}^{2}+z_{2} z_{7}+z_{7}^{2}-z_{3}-z_{6}-z_{1}-1 \\
& \chi_{1101000}=z_{1} z_{2} z_{4}-z_{1} z_{3} z_{5}-z_{2}^{2} z_{5}+z_{2} z_{3} z_{6}+z_{1} z_{5} z_{6}-z_{1} z_{4} z_{7}+z_{2} z_{6}^{2}-z_{2} z_{5} z_{7}-z_{1} z_{2} z_{7}^{2}-z_{6}^{2} z_{7}+z_{1}^{2} z_{5} \\
& -z_{3} z_{5}+z_{1} z_{2} z_{6}+z_{1} z_{7}^{3}+z_{5} z_{6}-z_{4} z_{7}-3 z_{1} z_{6} z_{7}+z_{7}^{3}+2 z_{1} z_{5}+2 z_{2} z_{6}-z_{6} z_{7}+z_{1} z_{2}+z_{5} \\
& -z_{1} z_{7}-z_{7} \\
& \chi_{0201000}=z_{2}^{2} z_{4}-z_{4}^{2}-z_{2} z_{3} z_{5}+z_{3}^{2} z_{6}+z_{1} z_{5}^{2}-2 z_{1} z_{4} z_{6}-z_{1}^{2} z_{6}^{2}+z_{3} z_{6}^{2}+z_{1}^{2} z_{5} z_{7}-z_{3} z_{5} z_{7}-z_{1}^{2} z_{4} \\
& +z_{1} z_{2} z_{6} z_{7}-z_{2}^{2} z_{7}^{2}-z_{5} z_{6} z_{7}+z_{3} z_{4}-z_{1} z_{2} z_{5}-z_{1} z_{3} z_{7}^{2}+z_{5}^{2}+z_{2}^{2} z_{6}+z_{4} z_{7}^{2}-z_{1}^{3} z_{6}+2 z_{1} z_{3} z_{6} \\
& +z_{1}^{2} z_{2} z_{7}+z_{1} z_{6} z_{7}^{2}-z_{1} z_{6}^{2}-z_{1} z_{2}^{2}+z_{1}^{2} z_{7}^{2}+z_{1} z_{4}+z_{2} z_{5}-z_{3} z_{7}^{2}-z_{6} z_{7}^{2}-z_{1}^{2} z_{6}+2 z_{3} z_{6}+z_{6}^{2} \\
& +z_{5} z_{7}+z_{2}^{2}-z_{1} z_{7}^{2}-z_{4}-z_{1}^{2}+z_{3}+z_{6}+z_{1} \\
& \chi_{1011000}=z_{1} z_{3} z_{4}-z_{4}^{2}-z_{1}^{2} z_{2} z_{5}+z_{1} z_{2}^{2} z_{6}+z_{1} z_{5}^{2}-z_{1} z_{4} z_{6}-z_{2} z_{4} z_{7}+z_{1}^{2} z_{5} z_{7}-2 z_{1} z_{2} z_{6} z_{7}-z_{1}^{2} z_{4} \\
& +z_{3} z_{4}-z_{5} z_{6} z_{7}+z_{1} z_{3} z_{7}^{2}+z_{1} z_{2} z_{5}-2 z_{1}^{2} z_{2} z_{7}+z_{4} z_{7}^{2}+z_{5}^{2}+z_{2} z_{7}^{3}+z_{1} z_{5} z_{7}+z_{1}^{2} z_{7}^{2}+2 z_{1} z_{2}^{2} \\
& -z_{3} z_{7}^{2}+z_{1} z_{3}-z_{4}-z_{1} z_{6}-z_{2} z_{7}-z_{1}^{2}+z_{3} \\
& \chi_{0111000}=z_{2} z_{3} z_{4}-z_{3}^{2} z_{5}-z_{1} z_{2}^{2} z_{5}+z_{1} z_{2} z_{3} z_{6}+z_{2} z_{5}^{2}+z_{1}^{2} z_{5} z_{6}-z_{3} z_{5} z_{6}-z_{1}^{2} z_{4} z_{7}-z_{5} z_{6}^{2}+z_{1} z_{2} z_{5} z_{7} \\
& -z_{1}^{3} z_{6} z_{7}+z_{1} z_{3} z_{6} z_{7}+2 z_{1}^{3} z_{5}-4 z_{1} z_{3} z_{5}-z_{2} z_{3} z_{7}^{2}+2 z_{4} z_{5}+z_{2} z_{3} z_{6}+z_{1}^{2} z_{7}^{3}-2 z_{1} z_{5} z_{6}+z_{1} z_{4} z_{7} \\
& +2 z_{2} z_{5} z_{7}-z_{3} z_{6} z_{7}-z_{1}^{2} z_{5}-z_{3} z_{5}-z_{5} z_{6}-z_{1} z_{7}^{3}+z_{2} z_{3}+z_{1} z_{6} z_{7}-3 z_{1} z_{5}-z_{1}^{2} z_{7}+z_{1} z_{7} \\
& \chi_{0021000}=z_{3}^{2} z_{4}-z_{1} z_{4}^{2}-z_{1} z_{2} z_{3} z_{5}+z_{2} z_{4} z_{5}+z_{1}^{2} z_{5}^{2}+z_{1}^{2} z_{2}^{2} z_{6}-z_{3} z_{5}^{2}-2 z_{1}^{2} z_{4} z_{6}-z_{2}^{2} z_{3} z_{6}+3 z_{3} z_{4} z_{6} \\
& -z_{2}^{2} z_{6}^{2}-z_{1}^{3} z_{6}^{2}-z_{5}^{2} z_{6}-z_{1} z_{2} z_{4} z_{7}+2 z_{1} z_{3} z_{6}^{2}+z_{1}^{3} z_{5} z_{7}+z_{2}^{2} z_{5} z_{7}-z_{1} z_{3} z_{5} z_{7}-2 z_{1}^{2} z_{2} z_{6} z_{7}-z_{1}^{3} z_{4}
\end{aligned}
$$


$+z_{4} z_{6}^{2}+z_{4} z_{5} z_{7}+z_{2} z_{3} z_{6} z_{7}+z_{1} z_{6}^{3}+2 z_{1} z_{3} z_{4}-z_{1} z_{5} z_{6} z_{7}+z_{1}^{2} z_{3} z_{7}^{2}+z_{1}^{2} z_{2} z_{5}-z_{1}^{4} z_{6}-2 z_{2} z_{3} z_{5}$

$-z_{3}^{2} z_{7}^{2}+z_{2} z_{6}^{2} z_{7}+z_{1} z_{4} z_{7}^{2}+3 z_{1}^{2} z_{3} z_{6}+z_{1}^{2} z_{6} z_{7}^{2}-z_{1} z_{5}^{2}-z_{1}^{3} z_{2} z_{7}-z_{3}^{2} z_{6}+z_{1} z_{4} z_{6}-2 z_{3} z_{6} z_{7}^{2}$

$-z_{6}^{2} z_{7}^{2}+z_{1}^{2} z_{2}^{2}-z_{2} z_{5} z_{6}+2 z_{1}^{2} z_{6}^{2}+z_{2} z_{4} z_{7}-z_{1}^{2} z_{5} z_{7}-z_{3} z_{6}^{2}+z_{1}^{3} z_{7}^{2}+z_{1} z_{2} z_{7}^{3}+z_{1}^{2} z_{4}+z_{1}^{3} z_{6}$

$+z_{1} z_{2} z_{6} z_{7}+z_{5} z_{6} z_{7}-3 z_{1} z_{3} z_{7}^{2}-2 z_{1} z_{2} z_{5}-2 z_{1} z_{6} z_{7}^{2}-z_{5}^{2}-z_{2} z_{7}^{3}-2 z_{2}^{2} z_{6}+z_{1} z_{6}^{2}+z_{2} z_{3} z_{7}$

$+z_{3}^{2}-z_{1} z_{4}+2 z_{2} z_{6} z_{7}-z_{1}^{2} z_{7}^{2}-2 z_{2} z_{5}-z_{1}^{3}+z_{3} z_{6}+2 z_{1} z_{3}-z_{2}^{2}+z_{1} z_{6}+z_{2} z_{7}+z_{1}^{2}$

$\chi_{1002000}$

$=z_{1} z_{4}^{2}-z_{1} z_{2} z_{3} z_{5}-z_{2} z_{4} z_{5}+z_{3} z_{5}^{2}+z_{1}^{2} z_{4} z_{6}+z_{2}^{2} z_{3} z_{6}-z_{3} z_{4} z_{6}-z_{1} z_{2} z_{4} z_{7}+z_{1}^{3} z_{5} z_{7}-z_{1} z_{3} z_{5} z_{7}$

$-z_{1}^{2} z_{2} z_{6} z_{7}-z_{1} z_{5} z_{6} z_{7}+z_{3}^{2} z_{7}^{2}-z_{1} z_{4} z_{7}^{2}+z_{2} z_{3} z_{5}+z_{2} z_{5} z_{7}^{2}-z_{3}^{2} z_{6}+2 z_{1} z_{4} z_{6}-z_{1} z_{2} z_{3} z_{7}+z_{1}^{2} z_{6}^{2}$

$+z_{1} z_{2} z_{7}^{3}-2 z_{1}^{2} z_{5} z_{7}+z_{3} z_{5} z_{7}-z_{1} z_{2} z_{6} z_{7}+z_{5} z_{6} z_{7}+z_{1}^{2} z_{4}+z_{2}^{2} z_{3}-2 z_{3} z_{4}+2 z_{1} z_{2} z_{5}+z_{1}^{3} z_{6}-z_{5}^{2}$

$-2 z_{1} z_{3} z_{6}-z_{1} z_{6} z_{7}^{2}+z_{1} z_{6}^{2}-2 z_{1} z_{5} z_{7}+z_{1} z_{4}-z_{1}^{2} z_{7}^{2}+2 z_{3} z_{7}^{2}+z_{1}^{2} z_{6}-2 z_{3} z_{6}-z_{1} z_{2} z_{7}+z_{1} z_{6}$

$+z_{1}^{2}-2 z_{3}$

$\chi_{0102000}=z_{2} z_{4}^{2}-z_{2}^{2} z_{3} z_{5}-z_{3} z_{4} z_{5}+z_{2} z_{3}^{2} z_{6}+z_{1} z_{2} z_{5}^{2}-z_{4} z_{5} z_{6}-z_{1} z_{3} z_{4} z_{7}-z_{1}^{2} z_{2} z_{6}^{2}+z_{4}^{2} z_{7}+2 z_{2} z_{3} z_{6}^{2}$

$+2 z_{1}^{2} z_{2} z_{5} z_{7}-z_{1} z_{5} z_{6}^{2}-3 z_{2} z_{3} z_{5} z_{7}-z_{1}^{2} z_{3} z_{6} z_{7}+2 z_{1} z_{4} z_{6} z_{7}+z_{1}^{2} z_{6}^{2} z_{7}+z_{3}^{2} z_{5}-z_{1} z_{4} z_{5}-2 z_{2} z_{4} z_{7}^{2}$

$-z_{2} z_{5}^{2}-z_{3} z_{5} z_{7}^{2}+2 z_{2} z_{4} z_{6}-3 z_{1}^{2} z_{5} z_{6}+4 z_{3} z_{5} z_{6}+2 z_{1}^{2} z_{4} z_{7}+z_{5} z_{6} z_{7}^{2}-z_{3} z_{4} z_{7}+z_{1} z_{3} z_{7}^{3}-2 z_{4} z_{7}^{3}$

$+2 z_{1}^{3} z_{6} z_{7}+z_{5} z_{6}^{2}-2 z_{1} z_{6} z_{7}^{3}-2 z_{5}^{2} z_{7}-4 z_{1} z_{3} z_{6} z_{7}+2 z_{4} z_{6} z_{7}-2 z_{1}^{3} z_{5}-2 z_{1} z_{2} z_{4}+2 z_{1} z_{6}^{2} z_{7}$

$+z_{2} z_{7}^{4}+5 z_{1} z_{3} z_{5}+z_{4} z_{5}-2 z_{1}^{2} z_{2} z_{6}+z_{7}^{5}+2 z_{2} z_{3} z_{6}-z_{1} z_{5} z_{7}^{2}-2 z_{2} z_{6} z_{7}^{2}+2 z_{1} z_{5} z_{6}+z_{2} z_{6}^{2}$

$-2 z_{1}^{2} z_{7}^{3}+z_{3} z_{7}^{3}+2 z_{1}^{2} z_{6} z_{7}-2 z_{6} z_{7}^{3}-z_{3} z_{6} z_{7}+2 z_{2} z_{4}+2 z_{1} z_{2} z_{7}^{2}+2 z_{3} z_{5}-2 z_{1} z_{2} z_{6}+z_{6}^{2} z_{7}$

$-z_{5} z_{7}^{2}-z_{1} z_{3} z_{7}+2 z_{5} z_{6}+2 z_{4} z_{7}+2 z_{1} z_{6} z_{7}-2 z_{2} z_{7}^{2}+z_{1} z_{5}+2 z_{1}^{2} z_{7}-2 z_{7}^{3}+2 z_{2} z_{6}-z_{3} z_{7}$

$+2 z_{6} z_{7}-2 z_{1} z_{2}+z_{5}+z_{2}+z_{7}$

$\chi_{0012000}=z_{3} z_{4}^{2}-z_{2} z_{3}^{2} z_{5}-z_{1} z_{2} z_{4} z_{5}+z_{1} z_{3} z_{5}^{2}+z_{2}^{2} z_{5}^{2}-z_{4} z_{5}^{2}+z_{1} z_{2}^{2} z_{3} z_{6}-z_{2}^{2} z_{4} z_{6}+2 z_{4}^{2} z_{6}-z_{2} z_{3} z_{5} z_{6}$

$-z_{1} z_{2}^{2} z_{6}^{2}-z_{1}^{2} z_{2} z_{4} z_{7}-z_{1}^{2} z_{3} z_{6}^{2}+z_{3}^{2} z_{6}^{2}-z_{1} z_{5}^{2} z_{6}+3 z_{1} z_{4} z_{6}^{2}+2 z_{1}^{2} z_{3} z_{5} z_{7}-2 z_{3}^{2} z_{5} z_{7}+z_{1} z_{2}^{2} z_{5} z_{7}$

$-z_{1} z_{4} z_{5} z_{7}-z_{1}^{3} z_{2} z_{6} z_{7}-z_{2} z_{5} z_{6}^{2}+z_{1}^{2} z_{6}^{3}+z_{2} z_{5}^{2} z_{7}+z_{3} z_{6}^{3}+z_{1} z_{4}^{2}+z_{2} z_{4} z_{6} z_{7}+z_{1} z_{3}^{2} z_{7}^{2}-z_{1}^{2} z_{5} z_{6} z_{7}$

$-2 z_{3} z_{5} z_{6} z_{7}+z_{1}^{2} z_{4} z_{7}^{2}-z_{1}^{2} z_{5}^{2}-z_{1} z_{3}^{2} z_{6}-z_{2} z_{4} z_{5}-3 z_{3} z_{4} z_{7}^{2}+3 z_{1}^{2} z_{4} z_{6}+z_{3} z_{5}^{2}+z_{3} z_{4} z_{6}+z_{1}^{3} z_{6} z_{7}^{2}$

$+z_{1} z_{2} z_{6}^{2} z_{7}+z_{5} z_{6}^{2} z_{7}+z_{1} z_{2} z_{5} z_{7}^{2}+z_{2}^{2} z_{6} z_{7}^{2}-2 z_{1} z_{3} z_{6} z_{7}^{2}-4 z_{4} z_{6} z_{7}^{2}-2 z_{1} z_{2} z_{5} z_{6}-z_{5}^{2} z_{6}-z_{1}^{2} z_{2} z_{3} z_{7}$

$-3 z_{1} z_{6}^{2} z_{7}^{2}+2 z_{1}^{3} z_{6}^{2}-z_{2}^{2} z_{6}^{2}+z_{1}^{2} z_{2} z_{7}^{3}-2 z_{1}^{3} z_{5} z_{7}-z_{1}^{2} z_{7}^{4}+2 z_{4} z_{6}^{2}+z_{1} z_{2} z_{4} z_{7}+z_{1} z_{6}^{3}+z_{1}^{3} z_{4}$

$+z_{1} z_{3} z_{5} z_{7}+z_{1} z_{2}^{2} z_{3}+2 z_{4} z_{5} z_{7}-z_{2} z_{3} z_{6} z_{7}-z_{2} z_{6} z_{7}^{3}-z_{2}^{2} z_{4}-2 z_{1} z_{3} z_{4}-z_{1}^{2} z_{3} z_{7}^{2}+4 z_{1} z_{5} z_{6} z_{7}$

$+z_{2} z_{6}^{2} z_{7}+2 z_{3} z_{7}^{4}+z_{3}^{2} z_{7}^{2}+2 z_{2} z_{3} z_{5}+z_{1}^{4} z_{6}-2 z_{1} z_{5}^{2}-2 z_{1} z_{4} z_{7}^{2}+2 z_{6} z_{7}^{4}-z_{1}^{2} z_{3} z_{6}-z_{3}^{2} z_{6}-z_{1} z_{2}^{2} z_{6}$

$-2 z_{1}^{2} z_{6} z_{7}^{2}+z_{1} z_{2} z_{3} z_{7}-2 z_{2} z_{5} z_{6}-z_{3} z_{6} z_{7}^{2}-2 z_{6}^{2} z_{7}^{2}+z_{1}^{2} z_{6}^{2}-z_{1} z_{2} z_{7}^{3}-2 z_{5} z_{7}^{3}+z_{2} z_{4} z_{7}-z_{1}^{3} z_{7}^{2}$

$+\quad z_{3} z_{5} z_{7}+2 z_{1} z_{2} z_{6} z_{7}-z_{1}^{2} z_{4}+3 z_{1} z_{3} z_{7}^{2}+z_{2}^{2} z_{7}^{2}+z_{3} z_{4}+3 z_{5} z_{6} z_{7}-z_{1} z_{2} z_{5}-z_{5}^{2}-2 z_{2}^{2} z_{6}$

$-2 z_{1} z_{3} z_{6}+z_{1} z_{7}^{4}+2 z_{4} z_{6}-z_{2} z_{7}^{3}-z_{1}^{2} z_{2} z_{7}-z_{2} z_{3} z_{7}+2 z_{1} z_{5} z_{7}+z_{1} z_{4}+2 z_{1}^{2} z_{7}^{2}+2 z_{2} z_{6} z_{7}+z_{1}^{2} z_{6}$

$-2 z_{3} z_{7}^{2}-z_{2} z_{5}-z_{3} z_{6}+z_{1}^{3}+z_{1} z_{2} z_{7}-2 z_{6} z_{7}^{2}-z_{2}^{2}+2 z_{5} z_{7}-z_{1} z_{7}^{2}-2 z_{1} z_{3}-z_{1} z_{6}+z_{2} z_{7}-z_{1}^{2}$

$\chi_{0003000}=z_{4}^{3}-2 z_{2} z_{3} z_{4} z_{5}+z_{3}^{2} z_{5}^{2}+z_{1} z_{2}^{2} z_{5}^{2}+z_{2}^{2} z_{3}^{2} z_{6}-z_{3}^{2} z_{4} z_{6}-z_{1} z_{2}^{2} z_{4} z_{6}-z_{1} z_{4} z_{5}^{2}+3 z_{1} z_{4}^{2} z_{6}$

$-z_{1} z_{2} z_{3} z_{5} z_{6}-z_{2} z_{4} z_{5} z_{6}-z_{1}^{2} z_{2}^{2} z_{6}^{2}-z_{1} z_{3}^{2} z_{6}^{2}-z_{1}^{2} z_{5}^{2} z_{6}-z_{1} z_{2} z_{3} z_{4} z_{7}+z_{3} z_{5}^{2} z_{6}+3 z_{1}^{2} z_{4} z_{6}^{2}+z_{2}^{2} z_{3} z_{6}^{2}$

$+z_{2} z_{4}^{2} z_{7}+z_{1}^{2} z_{2}^{2} z_{5} z_{7}+z_{1} z_{3}^{2} z_{5} z_{7}-z_{2}^{2} z_{3} z_{5} z_{7}-2 z_{3} z_{4} z_{5} z_{7}-z_{1} z_{2} z_{5} z_{6}^{2}-z_{1}^{2} z_{2} z_{3} z_{6} z_{7}+z_{1}^{3} z_{6}^{3}$

$+2 z_{1} z_{2} z_{4} z_{6} z_{7}+z_{3}^{3} z_{7}^{2}+z_{5}^{3} z_{7}-2 z_{1} z_{3} z_{5} z_{6} z_{7}+z_{1}^{2} z_{4}^{2}-z_{1}^{2} z_{2} z_{3} z_{5}-z_{3} z_{4}^{2}+2 z_{2} z_{3}^{2} z_{5}-z_{1} z_{2} z_{4} z_{5}$

$-z_{3}^{3} z_{6}-2 z_{1}^{3} z_{5}^{2}+5 z_{1} z_{3} z_{5}^{2}+z_{1}^{2} z_{2} z_{6}^{2} z_{7}+3 z_{1}^{3} z_{4} z_{6}-3 z_{4}^{2} z_{7}^{2}-4 z_{4} z_{5}^{2}-4 z_{1} z_{3} z_{4} z_{6}+z_{1} z_{2}^{2} z_{6} z_{7}^{2}$

$+z_{1} z_{5}^{2} z_{7}^{2}-z_{1} z_{2} z_{3}^{2} z_{7}+z_{3}^{2} z_{6} z_{7}^{2}+3 z_{4}^{2} z_{6}-2 z_{1}^{2} z_{2} z_{5} z_{6}-6 z_{1} z_{4} z_{6} z_{7}^{2}-3 z_{1}^{2} z_{6}^{2} z_{7}^{2}+2 z_{1}^{4} z_{6}^{2}+3 z_{2} z_{3} z_{5} z_{6}$

$-z_{1} z_{2}^{2} z_{6}^{2}+z_{1}^{2} z_{2} z_{4} z_{7}-z_{1} z_{5}^{2} z_{6}-3 z_{1}^{2} z_{3} z_{6}^{2}+z_{2} z_{5} z_{6} z_{7}^{2}-z_{1}^{4} z_{5} z_{7}+z_{1} z_{2} z_{3} z_{7}^{3}-z_{3}^{2} z_{6}^{2}+4 z_{1} z_{4} z_{6}^{2}$

$-2 z_{2} z_{4} z_{7}^{3}+z_{1}^{2} z_{3} z_{5} z_{7}+2 z_{3}^{2} z_{5} z_{7}+2 z_{3} z_{5} z_{7}^{3}+z_{1}^{2} z_{6}^{3}-2 z_{2} z_{5}^{2} z_{7}-2 z_{1} z_{2} z_{6} z_{7}^{3}+z_{1}^{3} z_{2} z_{6} z_{7}+z_{1}^{2} z_{3} z_{4}$

$-3 z_{1} z_{2} z_{3} z_{6} z_{7}-z_{1}^{3} z_{3} z_{7}^{2}+z_{2}^{2} z_{3}^{2}-z_{1}^{3} z_{2} z_{5}+2 z_{1}^{2} z_{5} z_{6} z_{7}-z_{1} z_{2}^{2} z_{4}-2 z_{3}^{2} z_{4}-2 z_{1} z_{4}^{2}+4 z_{1} z_{2} z_{3} z_{5}$ 
$+2 z_{1} z_{3}^{2} z_{7}^{2}+3 z_{2} z_{4} z_{6} z_{7}-z_{1}^{2} z_{2}^{2} z_{6}-2 z_{3} z_{5} z_{6} z_{7}-z_{1}^{2} z_{5}^{2}-2 z_{1}^{2} z_{4} z_{7}^{2}+2 z_{1}^{3} z_{3} z_{6}+3 z_{1} z_{2} z_{6}^{2} z_{7}$

$+2 z_{3} z_{4} z_{7}^{2}-4 z_{1} z_{3}^{2} z_{6}-3 z_{1}^{3} z_{6} z_{7}^{2}+3 z_{4} z_{7}^{4}-z_{2} z_{4} z_{5}-z_{1} z_{2} z_{5} z_{7}^{2}+2 z_{2}^{2} z_{3} z_{6}+6 z_{1} z_{3} z_{6} z_{7}^{2}+3 z_{1} z_{6} z_{7}^{4}$

$+z_{5}^{2} z_{7}^{2}+5 z_{3} z_{5}^{2}-2 z_{1}^{2} z_{4} z_{6}+z_{1}^{2} z_{2} z_{3} z_{7}-2 z_{3} z_{4} z_{6}-z_{1} z_{2} z_{5} z_{6}-5 z_{4} z_{6} z_{7}^{2}-z_{2} z_{3}^{2} z_{7}+z_{1}^{3} z_{6}^{2}$

$+z_{1}^{3} z_{5} z_{7}-3 z_{1} z_{6}^{2} z_{7}^{2}-6 z_{1} z_{3} z_{6}^{2}-z_{1}^{2} z_{2} z_{7}^{3}+z_{2} z_{7}^{5}+2 z_{4} z_{6}^{2}+2 z_{4} z_{5} z_{7}-z_{7}^{6}+z_{1}^{2} z_{2} z_{6} z_{7}-z_{1} z_{5} z_{7}^{3}$

$-2 z_{1} z_{3} z_{4}-z_{2} z_{3} z_{6} z_{7}+2 z_{1} z_{5} z_{6} z_{7}+z_{1} z_{2}^{2} z_{7}^{2}+z_{1}^{2} z_{7}^{4}-z_{1}^{2} z_{2} z_{5}+3 z_{4}^{2}+2 z_{3}^{2} z_{7}^{2}-3 z_{2} z_{6} z_{7}^{3}-z_{1}^{2} z_{3} z_{6}$

$+3 z_{2} z_{3} z_{5}-2 z_{3} z_{7}^{4}-2 z_{1} z_{2}^{2} z_{6}+2 z_{6} z_{7}^{4}-3 z_{3}^{2} z_{6}+3 z_{1} z_{5}^{2}+3 z_{1} z_{4} z_{7}^{2}+2 z_{2} z_{6}^{2} z_{7}+z_{1}^{2} z_{6} z_{7}^{2}+z_{2} z_{5} z_{7}^{2}$

$+4 z_{3} z_{6} z_{7}^{2}-z_{6}^{2} z_{7}^{2}-2 z_{1}^{2} z_{6}^{2}-3 z_{1} z_{2} z_{3} z_{7}-2 z_{3} z_{6}^{2}+3 z_{2} z_{4} z_{7}+3 z_{1}^{2} z_{5} z_{7}+z_{2}^{2} z_{3}-z_{5} z_{7}^{3}-4 z_{3} z_{5} z_{7}$

$-z_{1} z_{7}^{4}+2 z_{1}^{2} z_{4}+3 z_{1} z_{2} z_{6} z_{7}+3 z_{1}^{3} z_{6}-2 z_{3} z_{4}+3 z_{1} z_{3} z_{7}^{2}-8 z_{1} z_{3} z_{6}+z_{1}^{2} z_{2} z_{7}-5 z_{4} z_{7}^{2}+4 z_{4} z_{6}$

$-3 z_{2} z_{7}^{3}-2 z_{1} z_{6}^{2}+z_{1}^{2} z_{3}-z_{2} z_{3} z_{7}-z_{1} z_{2}^{2}+2 z_{7}^{4}+4 z_{2} z_{6} z_{7}-2 z_{1}^{2} z_{7}^{2}-2 z_{3}^{2}+4 z_{3} z_{7}^{2}-4 z_{1} z_{4}$

- $2 z_{1}^{2} z_{6}-2 z_{6} z_{7}^{2}-4 z_{3} z_{6}-2 z_{1} z_{3}+3 z_{1} z_{7}^{2}+2 z_{4}-4 z_{1} z_{6}+z_{1}^{2}+2 z_{2} z_{7}-2 z_{3}-z_{7}^{2}-2 z_{1}$

$\chi_{2000100}=z_{1}^{2} z_{5}-z_{3} z_{5}-z_{1} z_{2} z_{6}+z_{2}^{2} z_{7}-z_{4} z_{7}-z_{1} z_{5}+z_{3} z_{7}-2 z_{1} z_{2}+z_{1} z_{7}$

$\chi_{1100100}=z_{1} z_{2} z_{5}-z_{2}^{2} z_{6}-z_{1} z_{3} z_{6}-z_{5}^{2}+z_{4} z_{6}+z_{1} z_{6}^{2}+z_{2} z_{3} z_{7}-z_{1} z_{5} z_{7}+z_{2} z_{6} z_{7}+z_{1}^{2} z_{6}-z_{2} z_{5}$

$-z_{3} z_{6}-z_{6} z_{7}^{2}-z_{2}^{2}-z_{1} z_{7}^{2}+z_{2} z_{7}+z_{7}^{2}$

$\chi_{0200100}=z_{2}^{2} z_{5}-z_{4} z_{5}-z_{2} z_{3} z_{6}+z_{3}^{2} z_{7}-z_{2} z_{6}^{2}-z_{1} z_{4} z_{7}+z_{2} z_{5} z_{7}+z_{3} z_{6} z_{7}-z_{1}^{2} z_{5}-z_{1}^{3} z_{7}$

$+z_{1} z_{3} z_{7}+z_{1}^{2} z_{2}+z_{4} z_{7}-z_{2} z_{3}+z_{1} z_{6} z_{7}+z_{2} z_{7}^{2}+z_{1}^{2} z_{7}-2 z_{2} z_{6}-z_{1} z_{2}-z_{2}$

$\chi_{1010100}=z_{1} z_{3} z_{5}-z_{4} z_{5}-z_{1}^{2} z_{2} z_{6}+z_{1} z_{2}^{2} z_{7}+z_{2} z_{6}^{2}+z_{1}^{2} z_{6} z_{7}-z_{2} z_{5} z_{7}-z_{3} z_{6} z_{7}-z_{2} z_{4}-z_{6}^{2} z_{7}-z_{1}^{2} z_{5}$

$+2 z_{3} z_{5}-z_{1} z_{2} z_{6}+2 z_{5} z_{6}+z_{1} z_{3} z_{7}-z_{1} z_{7}^{3}-2 z_{1}^{2} z_{2}-z_{2}^{2} z_{7}+z_{1} z_{6} z_{7}+z_{2} z_{3}+z_{1} z_{5}+2 z_{2} z_{6}$

$+z_{1}^{2} z_{7}+z_{7}^{3}+z_{1} z_{2}-z_{6} z_{7}+z_{5}+z_{2}-z_{7}$

$\chi_{0110100}=z_{2} z_{3} z_{5}-z_{3}^{2} z_{6}-z_{1} z_{5}^{2}-z_{1} z_{2}^{2} z_{6}+z_{1} z_{4} z_{6}+z_{2} z_{5} z_{6}+z_{1} z_{2} z_{3} z_{7}+2 z_{1}^{2} z_{6}^{2}-2 z_{3} z_{6}^{2}$

$-2 z_{1}^{2} z_{5} z_{7}+2 z_{3} z_{5} z_{7}+z_{1} z_{2} z_{6} z_{7}-z_{6}^{3}+z_{5} z_{6} z_{7}-z_{3} z_{4}-z_{1}^{3} z_{7}^{2}+z_{1} z_{3} z_{7}^{2}+2 z_{1}^{3} z_{6}-4 z_{1} z_{3} z_{6}$

$-z_{1} z_{6} z_{7}^{2}-2 z_{1} z_{6}^{2}+2 z_{1} z_{5} z_{7}+z_{2} z_{6} z_{7}+z_{1}^{2} z_{7}^{2}-z_{1} z_{4}+z_{3} z_{7}^{2}-z_{1}^{2} z_{6}+2 z_{6} z_{7}^{2}-3 z_{3} z_{6}-3 z_{6}^{2}$

$+z_{1} z_{7}^{2}-3 z_{1} z_{6}-z_{3}-2 z_{6}-z_{1}$

$\chi_{0020100}=z_{3}^{2} z_{5}-z_{1} z_{4} z_{5}-z_{1} z_{2} z_{3} z_{6}+z_{2} z_{4} z_{6}+z_{1}^{2} z_{2}^{2} z_{7}+z_{1} z_{2} z_{6}^{2}-z_{5} z_{6}^{2}-z_{1}^{2} z_{4} z_{7}-z_{2}^{2} z_{3} z_{7}+2 z_{3} z_{4} z_{7}$

$-z_{1} z_{2} z_{5} z_{7}+z_{5}^{2} z_{7}-z_{2}^{2} z_{6} z_{7}-z_{1} z_{2} z_{4}-z_{1}^{3} z_{5}+z_{2}^{2} z_{5}-z_{1}^{2} z_{2} z_{7}^{2}+2 z_{4} z_{6} z_{7}+3 z_{1} z_{3} z_{5}+z_{2} z_{3} z_{7}^{2}$

$-2 z_{4} z_{5}-z_{2} z_{3} z_{6}-z_{1}^{4} z_{7}+z_{1} z_{5} z_{7}^{2}-z_{1} z_{2}^{2} z_{7}-z_{1} z_{5} z_{6}+4 z_{1}^{2} z_{3} z_{7}+z_{2} z_{6}^{2}-2 z_{3}^{2} z_{7}+z_{2}^{3}-z_{1}^{3} z_{2}$

$+2 z_{1}^{2} z_{6} z_{7}+z_{1} z_{2} z_{3}-z_{2} z_{4}+2 z_{1}^{3} z_{7}-3 z_{3} z_{6} z_{7}+z_{3} z_{5}-z_{6}^{2} z_{7}+z_{1} z_{2} z_{7}^{2}+z_{5} z_{7}^{2}-z_{2}^{2} z_{7}-3 z_{1} z_{3} z_{7}$

$-z_{5} z_{6}+2 z_{2} z_{3}+z_{1} z_{7}^{3}-4 z_{1} z_{6} z_{7}+z_{1} z_{5}+2 z_{2} z_{6}-z_{1}^{2} z_{7}-z_{3} z_{7}-z_{6} z_{7}+2 z_{1} z_{2}-z_{1} z_{7}$

$\chi_{1001100}=z_{1} z_{4} z_{5}-z_{2} z_{5}^{2}-z_{1} z_{2} z_{3} z_{6}+z_{3} z_{5} z_{6}+z_{1}^{2} z_{4} z_{7}+z_{1} z_{2} z_{6}^{2}+z_{2}^{2} z_{3} z_{7}-z_{3} z_{4} z_{7}+z_{5} z_{6}^{2}-z_{1} z_{2} z_{5} z_{7}$

$-z_{5}^{2} z_{7}+z_{1}^{3} z_{6} z_{7}-z_{1} z_{3} z_{6} z_{7}-z_{1}^{2} z_{2} z_{7}^{2}-z_{1} z_{2} z_{4}-z_{4} z_{6} z_{7}+z_{4} z_{5}-2 z_{1} z_{6}^{2} z_{7}+z_{2} z_{3} z_{7}^{2}-z_{2} z_{3} z_{6}$

$+3 z_{1} z_{5} z_{6}+z_{3}^{2} z_{7}-2 z_{1} z_{4} z_{7}+z_{6} z_{7}^{3}-2 z_{1}^{2} z_{6} z_{7}-z_{1} z_{2} z_{3}+z_{1}^{2} z_{5}+2 z_{3} z_{6} z_{7}+z_{2} z_{4}+z_{1} z_{2} z_{7}^{2}$

$-z_{3} z_{5}+z_{1} z_{2} z_{6}-z_{5} z_{7}^{2}+z_{5} z_{6}+z_{1}^{2} z_{2}+z_{4} z_{7}+z_{1} z_{7}^{3}-z_{1} z_{6} z_{7}-z_{2} z_{3}-z_{1} z_{2}-z_{7}^{3}-z_{1} z_{7}+z_{7}$

$\chi_{0101100}=z_{2} z_{4} z_{5}-z_{3} z_{5}^{2}-z_{2}^{2} z_{3} z_{6}+z_{1} z_{2} z_{5} z_{6}+z_{2} z_{3}^{2} z_{7}+z_{1} z_{3} z_{6}^{2}-z_{4} z_{6}^{2}-z_{1} z_{6}^{3}-z_{1} z_{3} z_{5} z_{7}-z_{1}^{2} z_{3} z_{7}^{2}$

$+z_{2} z_{3} z_{6} z_{7}-z_{1} z_{3} z_{4}+z_{4}^{2}+z_{1}^{2} z_{2} z_{5}+z_{1} z_{5} z_{6} z_{7}-2 z_{2} z_{3} z_{5}-z_{2} z_{5} z_{7}^{2}-z_{1} z_{5}^{2}+z_{1} z_{4} z_{6}-z_{1}^{2} z_{6}^{2}$

$+z_{6}^{2} z_{7}^{2}+z_{1} z_{2} z_{3} z_{7}+z_{3} z_{6}^{2}+z_{1} z_{2} z_{6} z_{7}-z_{2}^{2} z_{3}+z_{1}^{2} z_{4}+z_{1}^{3} z_{7}^{2}-z_{5} z_{6} z_{7}-z_{3} z_{4}-z_{4} z_{7}^{2}-z_{2}^{2} z_{6}$

$-z_{1} z_{6} z_{7}^{2}-z_{1} z_{3} z_{6}-z_{1}^{2} z_{2} z_{7}+z_{4} z_{6}+z_{1} z_{6}^{2}+z_{2} z_{3} z_{7}-2 z_{1}^{2} z_{7}^{2}+z_{1}^{2} z_{6}+z_{3} z_{7}^{2}-z_{3} z_{6}+2 z_{1} z_{2} z_{7}$

$-z_{1} z_{3}-z_{5} z_{7}-z_{2}^{2}+z_{4}+z_{1} z_{6}+z_{1}^{2}-z_{3}$

$\chi_{0011100}=z_{3} z_{4} z_{5}-z_{1} z_{2} z_{5}^{2}-z_{2} z_{3}^{2} z_{6}+z_{1} z_{3} z_{5} z_{6}+z_{2}^{2} z_{5} z_{6}+z_{1} z_{2}^{2} z_{3} z_{7}-z_{2}^{2} z_{4} z_{7}+z_{1}^{2} z_{2} z_{6}^{2}-z_{2} z_{3} z_{6}^{2}$

$+z_{4}^{2} z_{7}-z_{1}^{2} z_{2} z_{5} z_{7}-z_{2} z_{6}^{3}-z_{1}^{2} z_{2} z_{4}+z_{1}^{2} z_{3} z_{5}-z_{1} z_{2}^{2} z_{6} z_{7}+z_{1} z_{4} z_{6} z_{7}+z_{2} z_{5} z_{6} z_{7}+z_{1} z_{2}^{2} z_{5}$

$-z_{1}^{3} z_{2} z_{7}^{2}-z_{1}^{2} z_{6}^{2} z_{7}+z_{1} z_{2} z_{3} z_{7}^{2}+z_{3} z_{6}^{2} z_{7}-z_{3}^{2} z_{5}+z_{6}^{3} z_{7}+z_{1}^{2} z_{5} z_{7}^{2}-z_{1} z_{2} z_{3} z_{6}+z_{2} z_{4} z_{6}-z_{3} z_{5} z_{7}^{2}$ 
$+\quad z_{1} z_{2} z_{6} z_{7}^{2}+z_{2}^{2} z_{7}^{3}+z_{1}^{3} z_{7}^{3}+z_{1}^{2} z_{5} z_{6}+z_{1} z_{3}^{2} z_{7}-2 z_{3} z_{5} z_{6}-z_{1} z_{3} z_{7}^{3}+z_{1}^{2} z_{4} z_{7}-2 z_{3} z_{4} z_{7}-z_{5} z_{6} z_{7}^{2}$

$-z_{4} z_{7}^{3}-z_{1} z_{6} z_{7}^{3}-z_{1} z_{2} z_{5} z_{7}-z_{5} z_{6}^{2}+z_{5}^{2} z_{7}+z_{1}^{2} z_{2} z_{7}^{2}-z_{1}^{2} z_{2} z_{3}-z_{2}^{2} z_{6} z_{7}+z_{4} z_{6} z_{7}+2 z_{1} z_{6}^{2} z_{7}$

$+2 z_{1} z_{2} z_{4}+2 z_{2}^{2} z_{5}-z_{1} z_{3} z_{5}-2 z_{4} z_{5}-z_{2} z_{3} z_{7}^{2}+2 z_{1}^{2} z_{2} z_{6}-2 z_{2} z_{3} z_{6}-z_{2} z_{6} z_{7}^{2}-2 z_{1}^{2} z_{7}^{3}+z_{3} z_{7}^{3}$

$-z_{1}^{2} z_{3} z_{7}-3 z_{1} z_{5} z_{6}+z_{3}^{2} z_{7}-2 z_{2} z_{6}^{2}+z_{1}^{3} z_{2}-z_{1} z_{4} z_{7}-2 z_{1}^{2} z_{5}+z_{3} z_{6} z_{7}+z_{2} z_{4}-z_{1}^{3} z_{7}+z_{3} z_{5}$

$+z_{6}^{2} z_{7}+2 z_{1} z_{3} z_{7}-z_{5} z_{6}-z_{2}^{2} z_{7}+z_{4} z_{7}+z_{1} z_{7}^{3}+z_{1} z_{6} z_{7}-z_{2} z_{7}^{2}-z_{1}^{2} z_{2}+z_{1} z_{5}+2 z_{1}^{2} z_{7}-z_{3} z_{7}$

$-z_{1} z_{7}+z_{2}$

$\chi_{0002100}$

$=z_{4}^{2} z_{5}-z_{2} z_{3} z_{5}^{2}-z_{2} z_{3} z_{4} z_{6}+z_{3}^{2} z_{5} z_{6}+z_{1} z_{2}^{2} z_{5} z_{6}+z_{2}^{2} z_{3}^{2} z_{7}-z_{3}^{2} z_{4} z_{7}-z_{1} z_{2}^{2} z_{4} z_{7}-z_{2} z_{4} z_{6}^{2}$

$+2 z_{1} z_{4}^{2} z_{7}-z_{1} z_{2} z_{3} z_{5} z_{7}-z_{1}^{2} z_{5} z_{6}^{2}+2 z_{3} z_{5} z_{6}^{2}-z_{1} z_{3}^{2} z_{6} z_{7}-z_{1}^{2} z_{2}^{2} z_{6} z_{7}+z_{1}^{2} z_{5}^{2} z_{7}-z_{1} z_{2} z_{6}^{3}$

$-z_{1} z_{2} z_{3} z_{4}+z_{2} z_{4}^{2}-2 z_{3} z_{5}^{2} z_{7}+z_{2}^{2} z_{3} z_{6} z_{7}+3 z_{1}^{2} z_{4} z_{6} z_{7}+z_{1} z_{3}^{2} z_{5}-z_{3} z_{4} z_{6} z_{7}-z_{1}^{2} z_{2} z_{3} z_{7}^{2}+z_{1}^{2} z_{2}^{2} z_{5}$

$+z_{2} z_{3}^{2} z_{7}^{2}+z_{5}^{2} z_{6} z_{7}-z_{2}^{2} z_{3} z_{5}-z_{1}^{2} z_{4} z_{5}+z_{1}^{3} z_{6}^{2} z_{7}-z_{1} z_{3} z_{6}^{2} z_{7}+z_{1} z_{2} z_{4} z_{7}^{2}+z_{1}^{3} z_{5} z_{7}^{2}-2 z_{1} z_{3} z_{5} z_{7}^{2}$

$+z_{4} z_{6}^{2} z_{7}-z_{2} z_{3}^{2} z_{6}-z_{5}^{3}+z_{1} z_{2} z_{4} z_{6}+z_{3}^{3} z_{7}-3 z_{4} z_{5} z_{7}^{2}-3 z_{1}^{3} z_{5} z_{6}+z_{1}^{2} z_{2} z_{6} z_{7}^{2}+6 z_{1} z_{3} z_{5} z_{6}$

$+z_{1} z_{6}^{3} z_{7}-z_{4} z_{5} z_{6}+z_{2} z_{3} z_{6} z_{7}^{2}+2 z_{1}^{3} z_{4} z_{7}+z_{1} z_{2}^{2} z_{7}^{3}+z_{1}^{2} z_{3} z_{7}^{3}-2 z_{1} z_{5} z_{6} z_{7}^{2}+z_{2} z_{6}^{2} z_{7}^{2}-4 z_{1} z_{3} z_{4} z_{7}$

$-4 z_{1} z_{4} z_{7}^{3}-z_{2} z_{3} z_{6}^{2}-3 z_{1}^{2} z_{6} z_{7}^{3}-z_{1}^{2} z_{2} z_{5} z_{7}+z_{4}^{2} z_{7}+z_{1} z_{5} z_{6}^{2}+z_{2} z_{3} z_{5} z_{7}+2 z_{1}^{4} z_{6} z_{7}-z_{1} z_{5}^{2} z_{7}$

- $6 z_{1}^{2} z_{3} z_{6} z_{7}-z_{1} z_{2} z_{3}^{2}-z_{1} z_{2}^{2} z_{6} z_{7}+z_{1}^{2} z_{2} z_{4}-2 z_{1}^{4} z_{5}-z_{2} z_{3} z_{4}-z_{6}^{2} z_{7}^{3}-z_{2} z_{6}^{3}+2 z_{3}^{2} z_{6} z_{7}$

$+5 z_{1} z_{4} z_{6} z_{7}+z_{1} z_{2}^{2} z_{5}+5 z_{1}^{2} z_{3} z_{5}+3 z_{1}^{2} z_{6}^{2} z_{7}+z_{1}^{3} z_{2} z_{6}-z_{1} z_{2} z_{7}^{4}-z_{1}^{2} z_{5} z_{7}^{2}-3 z_{1} z_{4} z_{5}+z_{6}^{3} z_{7}$

$-z_{2} z_{4} z_{7}^{2}+2 z_{3} z_{5} z_{7}^{2}+2 z_{5} z_{7}^{4}-2 z_{1}^{3} z_{7}^{3}+z_{1} z_{2} z_{6} z_{7}^{2}-2 z_{1} z_{2} z_{3} z_{6}-z_{1}^{3} z_{3} z_{7}-z_{2} z_{4} z_{6}+2 z_{3} z_{5} z_{6}$

$+2 z_{1} z_{3}^{2} z_{7}+z_{2}^{2} z_{3} z_{7}-2 z_{1} z_{2} z_{6}^{2}-2 z_{1}^{2} z_{4} z_{7}-z_{1} z_{2} z_{5} z_{7}+2 z_{1} z_{7}^{5}+3 z_{1} z_{3} z_{7}^{3}-2 z_{5} z_{6} z_{7}^{2}-z_{4} z_{7}^{3}$

$+2 z_{1}^{2} z_{2} z_{3}-4 z_{1} z_{6} z_{7}^{3}+z_{5}^{2} z_{7}-2 z_{2} z_{3}^{2}-z_{1}^{2} z_{2} z_{7}^{2}-z_{1} z_{2} z_{4}+2 z_{4} z_{6} z_{7}+3 z_{1} z_{6}^{2} z_{7}+2 z_{1} z_{3} z_{5}$

$+3 z_{2} z_{3} z_{7}^{2}+z_{4} z_{5}+2 z_{1} z_{5} z_{7}^{2}-5 z_{2} z_{3} z_{6}+2 z_{1} z_{5} z_{6}+2 z_{2} z_{6} z_{7}^{2}-z_{1} z_{2}^{2} z_{7}+3 z_{1} z_{4} z_{7}-3 z_{2} z_{6}^{2}$

$=$ $2 z_{1}^{2} z_{7}^{3}-z_{3} z_{7}^{3}-2 z_{1} z_{2} z_{3}-z_{6} z_{7}^{3}+3 z_{1}^{2} z_{5}+z_{3} z_{6} z_{7}+2 z_{6}^{2} z_{7}-2 z_{3} z_{5}+2 z_{1} z_{2} z_{7}^{2}-2 z_{1} z_{2} z_{6}$ $2 z_{5} z_{7}^{2}+2 z_{1}^{3} z_{7}-2 z_{1} z_{3} z_{7}+z_{4} z_{7}+z_{1}^{2} z_{2}-3 z_{1} z_{7}^{3}+3 z_{1} z_{6} z_{7}-4 z_{2} z_{3}+z_{2} z_{7}^{2}-z_{1} z_{5}-3 z_{2} z_{6}$ $2 z_{1}^{2} z_{7}+z_{3} z_{7}-z_{1} z_{2}+z_{6} z_{7}+z_{1} z_{7}-z_{2}$

$-z_{1} z_{2} z_{5}+z_{4} z_{7}^{2}+z_{5}^{2}-z_{2} z_{7}^{3}-z_{4} z_{6}-z_{2} z_{3} z_{7}+z_{1} z_{5} z_{7}+z_{2} z_{6} z_{7}+z_{3}^{2}+z_{1}^{2} z_{7}^{2}-z_{3} z_{7}^{2}-2 z_{1} z_{4}$

$-2 z_{1}^{2} z_{6}-z_{2} z_{5}-z_{6} z_{7}^{2}+2 z_{3} z_{6}-z_{1}^{3}+2 z_{6}^{2}+2 z_{1} z_{3}+z_{5} z_{7}+z_{1} z_{7}^{2}-2 z_{4}-z_{1} z_{6}+z_{2} z_{7}+z_{3}-1$

$\chi_{0100200}=z_{2} z_{5}^{2}-z_{2} z_{4} z_{6}-z_{3} z_{5} z_{6}+z_{3} z_{4} z_{7}-z_{5} z_{6}^{2}+z_{1} z_{3} z_{6} z_{7}+z_{5}^{2} z_{7}-z_{1}^{2} z_{2} z_{7}^{2}+z_{2} z_{3} z_{7}^{2}+z_{1} z_{5} z_{7}^{2}$

$-z_{1} z_{3} z_{5}+z_{1}^{2} z_{2} z_{6}-z_{2} z_{3} z_{6}-z_{3}^{2} z_{7}-2 z_{1} z_{5} z_{6}+z_{1} z_{4} z_{7}+z_{2} z_{5} z_{7}-z_{3} z_{6} z_{7}+z_{1} z_{2} z_{3}-2 z_{2} z_{4}$

$+z_{1} z_{2} z_{7}^{2}+z_{5} z_{7}^{2}-z_{3} z_{5}+z_{1} z_{7}^{3}-2 z_{5} z_{6}+z_{1}^{3} z_{7}-2 z_{1} z_{3} z_{7}-z_{4} z_{7}-3 z_{1} z_{6} z_{7}+z_{2} z_{7}^{2}-z_{1} z_{5}$

$-2 z_{1}^{2} z_{7}-z_{2} z_{6}+z_{1} z_{2}-z_{5}-z_{1} z_{7}-z_{2}$

$\chi_{0010200}$

$=z_{3} z_{5}^{2}-z_{3} z_{4} z_{6}-z_{1} z_{2} z_{5} z_{6}+z_{2}^{2} z_{6}^{2}+$

$-z_{2} z_{3} z_{6} z_{7}-z_{1}^{2} z_{3} z_{7}^{2}-z_{1} z_{2}^{2} z_{7}^{2}-z_{4}^{2}-z_{1}^{2} z_{2} z_{5}+z_{3}^{2} z_{7}^{2}+z_{2} z_{3} z_{5}+2 z_{1} z_{4} z_{7}^{2}-z_{2} z_{6}^{2} z_{7}+z_{1} z_{2}^{2} z_{6}$

$+z_{1}^{2} z_{3} z_{6}-z_{3}^{2} z_{6}+z_{2} z_{5} z_{7}^{2}+z_{1} z_{5}^{2}-4 z_{1} z_{4} z_{6}+z_{3} z_{6} z_{7}^{2}+z_{2} z_{5} z_{6}-2 z_{1}^{2} z_{6}^{2}-z_{2} z_{4} z_{7}+3 z_{1}^{2} z_{5} z_{7}+z_{6}^{3}$

$+z_{2}^{2} z_{3}+z_{1} z_{3}^{2}-2 z_{3} z_{5} z_{7}+2 z_{1}^{3} z_{7}^{2}-2 z_{1}^{2} z_{4}-z_{1} z_{2} z_{6} z_{7}-z_{3} z_{4}+2 z_{2}^{2} z_{6}-z_{1} z_{3} z_{7}^{2}-3 z_{1}^{3} z_{6}-z_{5} z_{6} z_{7}$

$+2 z_{1} z_{3} z_{6}+z_{2} z_{7}^{3}-z_{1}^{4}-2 z_{4} z_{6}+z_{1} z_{6}^{2}-z_{2} z_{3} z_{7}+z_{1} z_{2}^{2}-2 z_{1} z_{5} z_{7}-2 z_{1}^{2} z_{7}^{2}+z_{1}^{2} z_{3}+z_{3}^{2}$

$-3 z_{2} z_{6} z_{7}+z_{3} z_{7}^{2}-z_{1} z_{4}+z_{2} z_{5}+2 z_{3} z_{6}+2 z_{6}^{2}-z_{5} z_{7}+z_{2}^{2}+2 z_{1} z_{3}+3 z_{1} z_{6}-2 z_{2} z_{7}+z_{1}^{2}+z_{6}$

$\chi_{0001200}=z_{4} z_{5}^{2}-z_{4}^{2} z_{6}-z_{2} z_{3} z_{5} z_{6}+z_{3}^{2} z_{6}^{2}+z_{2} z_{3} z_{4} z_{7}-z_{3}^{2} z_{5} z_{7}+z_{1} z_{2}^{2} z_{6}^{2}-2 z_{1} z_{4} z_{6}^{2}-z_{1} z_{2}^{2} z_{5} z_{7}$

$+3 z_{1} z_{4} z_{5} z_{7}-z_{2} z_{4} z_{6} z_{7}-z_{1}^{2} z_{2}^{2} z_{7}^{2}-z_{1} z_{4}^{2}-z_{1}^{2} z_{6}^{3}+z_{3} z_{6}^{3}-z_{1} z_{3}^{2} z_{7}^{2}+2 z_{1}^{2} z_{5} z_{6} z_{7}-z_{3} z_{5} z_{6} z_{7}$

$+z_{1} z_{3}^{2} z_{6}+z_{1}^{2} z_{4} z_{7}^{2}-2 z_{1} z_{2} z_{6}^{2} z_{7}+z_{2} z_{4} z_{5}+z_{2}^{2} z_{3} z_{7}^{2}+z_{3} z_{4} z_{7}^{2}-z_{3} z_{5}^{2}+z_{1}^{2} z_{2}^{2} z_{6}+z_{5} z_{6}^{2} z_{7}$

$+z_{1} z_{2} z_{5} z_{7}^{2}-z_{5}^{2} z_{7}^{2}-z_{2}^{2} z_{3} z_{6}+z_{1}^{3} z_{6} z_{7}^{2}-z_{1} z_{3} z_{6} z_{7}^{2}-3 z_{1}^{2} z_{4} z_{6}+z_{1}^{2} z_{2} z_{7}^{3}+z_{1}^{2} z_{2} z_{3} z_{7}+2 z_{1} z_{2} z_{5} z_{6}$ 
$-z_{2} z_{3}^{2} z_{7}+z_{4} z_{6} z_{7}^{2}-z_{5}^{2} z_{6}-3 z_{1}^{3} z_{6}^{2}+z_{3}^{3}+4 z_{1} z_{3} z_{6}^{2}+2 z_{1}^{3} z_{5} z_{7}+z_{1} z_{6}^{2} z_{7}^{2}-4 z_{1} z_{3} z_{5} z_{7}-z_{4} z_{6}^{2}$

$+z_{4} z_{5} z_{7}-z_{1}^{2} z_{2} z_{6} z_{7}-2 z_{1} z_{5} z_{7}^{3}-z_{1}^{3} z_{4}-z_{1}^{2} z_{7}^{4}+z_{1} z_{6}^{3}+z_{1}^{4} z_{7}^{2}-z_{1} z_{5} z_{6} z_{7}-z_{1}^{2} z_{3} z_{7}^{2}+z_{2} z_{6} z_{7}^{3}$

$+z_{1}^{2} z_{2} z_{5}-z_{2} z_{6}^{2} z_{7}-z_{3}^{2} z_{7}^{2}-z_{4}^{2}-2 z_{1}^{4} z_{6}+z_{1} z_{4} z_{7}^{2}-z_{2} z_{3} z_{5}+2 z_{1}^{2} z_{6} z_{7}^{2}-z_{1} z_{5}^{2}+z_{1} z_{2}^{2} z_{6}$

$+2 z_{1}^{2} z_{3} z_{6}+2 z_{3}^{2} z_{6}-2 z_{3} z_{6} z_{7}^{2}-z_{1} z_{4} z_{6}-2 z_{1} z_{2} z_{3} z_{7}-z_{6}^{2} z_{7}^{2}+z_{1}^{2} z_{6}^{2}+3 z_{3} z_{6}^{2}$

$+z_{1}^{2} z_{2}^{2}+z_{6}^{3}-2 z_{1}^{3} z_{3}-3 z_{1}^{2} z_{5} z_{7}+4 z_{1} z_{3}^{2}-2 z_{1} z_{2} z_{6} z_{7}-z_{2}^{2} z_{3}+z_{2}^{2} z_{7}^{2}-z_{1}^{3} z_{7}^{2}-z_{1} z_{3} z_{7}^{2}$

$+z_{1}^{2} z_{4}-z_{3} z_{4}+z_{4} z_{7}^{2}+6 z_{1} z_{3} z_{6}-z_{1} z_{6} z_{7}^{2}-z_{2}^{2} z_{6}-z_{4} z_{6}+z_{1}^{2} z_{3}-z_{2} z_{3} z_{7}+4 z_{1} z_{6}^{2}$

$+2 z_{3}^{2}-z_{2} z_{6} z_{7}+z_{1}^{2} z_{7}^{2}+2 z_{1}^{2} z_{6}-z_{3} z_{7}^{2}-z_{1}^{3}-z_{2} z_{5}-z_{6} z_{7}^{2}+3 z_{3} z_{6}-2 z_{1} z_{2} z_{7}+2 z_{6}^{2}$

$+4 z_{1} z_{3}-z_{1} z_{7}^{2}-z_{2}^{2}-z_{4}+3 z_{1} z_{6}+z_{1}^{2}+z_{3}+z_{6}+z_{1}$

$\chi_{0000300}$

$=z_{5}^{3}-2 z_{4} z_{5} z_{6}+z_{4}^{2} z_{7}+z_{2} z_{3} z_{6}^{2}-2 z_{1} z_{5} z_{6}^{2}-z_{2} z_{3} z_{5} z_{7}+z_{2} z_{6}^{3}+2 z_{1} z_{5}^{2} z_{7}+z_{1} z_{4} z_{6} z_{7}-2 z_{2} z_{5} z_{6} z_{7}$

$-z_{1} z_{2} z_{3} z_{7}^{2}+z_{2} z_{4} z_{7}^{2}-z_{1} z_{4} z_{5}+z_{1} z_{2} z_{3} z_{6}+z_{1}^{2} z_{5} z_{7}^{2}+z_{3} z_{5} z_{7}^{2}+z_{2} z_{5}^{2}-z_{2} z_{4} z_{6}-z_{1} z_{2} z_{6} z_{7}^{2}$

$+z_{5} z_{6} z_{7}^{2}-2 z_{1}^{2} z_{5} z_{6}+z_{1}^{2} z_{4} z_{7}+z_{1} z_{2} z_{6}^{2}-2 z_{3} z_{4} z_{7}+z_{1} z_{2} z_{5} z_{7}+z_{1} z_{3} z_{7}^{3}+z_{1}^{3} z_{6} z_{7}+z_{2} z_{3}^{2}$

$-z_{1} z_{2} z_{4}-z_{4} z_{7}^{3}-4 z_{1} z_{3} z_{6} z_{7}-z_{1}^{3} z_{5}+z_{1}^{2} z_{2} z_{7}^{2}+z_{4} z_{6} z_{7}-z_{1} z_{6}^{2} z_{7}+2 z_{1} z_{3} z_{5}-3 z_{4} z_{5}-z_{2} z_{3} z_{7}^{2}$

$-z_{1}^{2} z_{2} z_{6}+3 z_{2} z_{3} z_{6}+z_{1} z_{5} z_{7}^{2}-z_{1} z_{5} z_{6}-z_{2} z_{6} z_{7}^{2}+3 z_{2} z_{6}^{2}-3 z_{1} z_{4} z_{7}-z_{1}^{3} z_{2}+3 z_{1} z_{2} z_{3}+z_{3} z_{7}^{3}$

$-2 z_{1}^{2} z_{6} z_{7}+z_{6} z_{7}^{3}-2 z_{3} z_{6} z_{7}-2 z_{6}^{2} z_{7}-2 z_{2} z_{4}-z_{1} z_{2} z_{7}^{2}-z_{1}^{2} z_{5}-z_{1}^{3} z_{7}+2 z_{3} z_{5}+2 z_{1} z_{2} z_{6}+z_{5} z_{6}$

$+2 z_{4} z_{7}+z_{1} z_{7}^{3}+2 z_{2} z_{3}-z_{1} z_{6} z_{7}+2 z_{1} z_{5}+2 z_{2} z_{6}+2 z_{1}^{2} z_{7}-2 z_{3} z_{7}+2 z_{1} z_{2}-2 z_{6} z_{7}-2 z_{1} z_{7}$

$\chi_{2000010}=z_{1}^{2} z_{6}-z_{3} z_{6}-z_{1} z_{2} z_{7}+z_{2}^{2}-z_{6}^{2}+z_{5} z_{7}-z_{4}-z_{1} z_{6}+z_{2} z_{7}+z_{7}^{2}-2 z_{6}-1$

$\chi_{1100010}=z_{1} z_{2} z_{6}-z_{2}^{2} z_{7}-z_{1} z_{3} z_{7}-z_{5} z_{6}+z_{4} z_{7}+z_{2} z_{3}+z_{1}^{2} z_{7}+z_{2} z_{6}-z_{3} z_{7}+z_{1} z_{2}-z_{6} z_{7}-2 z_{1} z_{7}+z_{2}$

$\chi_{0200010}=z_{2}^{2} z_{6}-z_{4} z_{6}-z_{2} z_{3} z_{7}+z_{3}^{2}-z_{1} z_{6}^{2}+z_{1} z_{5} z_{7}-z_{2} z_{6} z_{7}+z_{1}^{2} z_{7}^{2}-z_{1} z_{4}+z_{2} z_{5}-2 z_{1}^{2} z_{6}+2 z_{3} z_{6}$

$-z_{1} z_{2} z_{7}-z_{1}^{3}+z_{6}^{2}+z_{2}^{2}+2 z_{1} z_{3}+z_{1} z_{6}+z_{3}+z_{6}+z_{1}$

$\chi_{1010010}=z_{1} z_{3} z_{6}-z_{4} z_{6}-z_{1} z_{6}^{2}-z_{1}^{2} z_{2} z_{7}+z_{1} z_{5} z_{7}+z_{1}^{2} z_{7}^{2}+z_{1} z_{2}^{2}+z_{2} z_{6} z_{7}-z_{1}^{2} z_{6}-z_{2} z_{5}-z_{3} z_{7}^{2}+z_{3} z_{6}$

$+z_{1} z_{2} z_{7}-z_{2}^{2}-z_{1} z_{7}^{2}$

$\chi_{0110010}=z_{2} z_{3} z_{6}-z_{1} z_{5} z_{6}-z_{3}^{2} z_{7}-z_{1} z_{2}^{2} z_{7}+z_{1} z_{4} z_{7}+z_{1} z_{2} z_{3}+z_{2} z_{5} z_{7}+z_{1}^{2} z_{6} z_{7}-z_{3} z_{6} z_{7}+z_{1} z_{2} z_{7}^{2}$

$-z_{1}^{2} z_{5}+z_{3} z_{5}+z_{1}^{3} z_{7}-z_{1} z_{7}^{3}-3 z_{1} z_{3} z_{7}+z_{4} z_{7}+z_{2} z_{3}-z_{1} z_{6} z_{7}+z_{2} z_{7}^{2}-z_{1}^{2} z_{7}-z_{3} z_{7}$

$\chi_{0020010}=z_{3}^{2} z_{6}-z_{1} z_{4} z_{6}-z_{1}^{2} z_{6}^{2}-z_{1} z_{2} z_{3} z_{7}+z_{2} z_{4} z_{7}+z_{3} z_{6}^{2}+z_{1}^{2} z_{5} z_{7}+z_{1}^{2} z_{2}^{2}-z_{3} z_{5} z_{7}+z_{1} z_{2} z_{6} z_{7}-z_{1}^{2} z_{4}$

$-z_{2}^{2} z_{3}+z_{1}^{3} z_{7}^{2}+2 z_{3} z_{4}-z_{1} z_{2} z_{5}-2 z_{1} z_{3} z_{7}^{2}+z_{4} z_{7}^{2}-2 z_{1}^{3} z_{6}-z_{2}^{2} z_{6}+4 z_{1} z_{3} z_{6}-z_{2} z_{7}^{3}-z_{1}^{4}+z_{1} z_{6}^{2}$

$+3 z_{1}^{2} z_{3}-z_{1} z_{5} z_{7}-z_{1} z_{2}^{2}+2 z_{2} z_{6} z_{7}-z_{1}^{2} z_{7}^{2}-z_{3}^{2}+2 z_{1}^{2} z_{6}-z_{2} z_{5}+z_{1}^{3}-z_{2}^{2}-z_{4}+z_{2} z_{7}$

$\chi_{1001010}=z_{1} z_{4} z_{6}-z_{2} z_{5} z_{6}-z_{1} z_{2} z_{3} z_{7}+z_{3} z_{5} z_{7}+z_{1}^{2} z_{4}+z_{1} z_{2} z_{6} z_{7}+z_{2}^{2} z_{3}+z_{1}^{3} z_{7}^{2}+z_{6}^{3}-z_{3} z_{4}-z_{5} z_{6} z_{7}$

$-z_{1} z_{3} z_{7}^{2}-z_{1} z_{2} z_{5}-2 z_{1} z_{6} z_{7}^{2}-z_{4} z_{6}-z_{1}^{2} z_{2} z_{7}+z_{1} z_{6}^{2}+z_{2} z_{3} z_{7}+z_{1} z_{5} z_{7}-2 z_{1}^{2} z_{7}^{2}-z_{2} z_{6} z_{7}$

$+z_{3} z_{7}^{2}-z_{1} z_{4}+z_{3} z_{6}+z_{6} z_{7}^{2}+z_{6}^{2}+z_{1} z_{2} z_{7}+z_{1} z_{7}^{2}+2 z_{1} z_{6}+z_{1}^{2}-z_{3}-z_{6}-z_{1}$

$\chi_{0101010}=z_{2} z_{4} z_{6}-z_{3} z_{5} z_{6}-z_{2}^{2} z_{3} z_{7}+z_{1} z_{2} z_{5} z_{7}+z_{2} z_{3}^{2}+z_{1} z_{3} z_{6} z_{7}-z_{4} z_{6} z_{7}+z_{1}^{2} z_{2} z_{7}^{2}-z_{2} z_{3} z_{7}^{2}-z_{1} z_{3} z_{5}$

$-z_{1}^{2} z_{7}^{3}-z_{1}^{2} z_{2} z_{6}+2 z_{2} z_{3} z_{6}-z_{2} z_{6} z_{7}^{2}-z_{1}^{2} z_{3} z_{7}-z_{1} z_{5} z_{6}+z_{2} z_{6}^{2}+z_{6} z_{7}^{3}-z_{2} z_{5} z_{7}+z_{3} z_{6} z_{7}$

$+z_{1} z_{2} z_{7}^{2}+z_{1} z_{2} z_{3}-z_{6}^{2} z_{7}-z_{1}^{2} z_{5}+z_{2} z_{4}-z_{5} z_{7}^{2}+z_{3} z_{5}+2 z_{5} z_{6}+z_{1}^{3} z_{7}+z_{1} z_{7}^{3}-z_{1} z_{3} z_{7}-2 z_{1}^{2} z_{2}$

$-z_{2}^{2} z_{7}+z_{4} z_{7}+2 z_{2} z_{3}+2 z_{1} z_{5}-2 z_{2} z_{7}^{2}+3 z_{2} z_{6}+z_{1} z_{2}-z_{7}^{3}+z_{5}-z_{1} z_{7}+2 z_{2}+z_{7}$

$\chi_{0011010}=z_{3} z_{4} z_{6}-z_{1} z_{2} z_{5} z_{6}-z_{2} z_{3}^{2} z_{7}+z_{2}^{2} z_{5} z_{7}+z_{1} z_{3} z_{5} z_{7}+z_{4} z_{6}^{2}-z_{4} z_{5} z_{7}+z_{1}^{2} z_{2} z_{6} z_{7}+z_{1} z_{2}^{2} z_{3}+z_{1} z_{6}^{3}$

$-z_{2}^{2} z_{4}+z_{1}^{2} z_{3} z_{7}^{2}-z_{2} z_{3} z_{6} z_{7}-z_{1} z_{5} z_{6} z_{7}+z_{4}^{2}-z_{1}^{2} z_{2} z_{5}-z_{3}^{2} z_{7}^{2}-z_{1} z_{4} z_{7}^{2}-z_{1} z_{2}^{2} z_{6}-2 z_{1}^{2} z_{6} z_{7}^{2}$

$-z_{1}^{2} z_{3} z_{6}+z_{3}^{2} z_{6}-z_{2} z_{6}^{2} z_{7}+z_{2} z_{5} z_{7}^{2}+2 z_{1} z_{4} z_{6}-z_{1}^{3} z_{2} z_{7}+2 z_{1}^{2} z_{6}^{2}+z_{1} z_{2} z_{3} z_{7}+z_{1}^{2} z_{5} z_{7}-z_{3} z_{5} z_{7}$

$+z_{2}^{2} z_{7}^{2}+z_{1} z_{7}^{4}+z_{3} z_{4}-z_{1} z_{2} z_{5}+z_{1}^{3} z_{6}+z_{1}^{2} z_{2} z_{7}+z_{4} z_{6}-2 z_{2} z_{3} z_{7}-2 z_{2} z_{6} z_{7}-2 z_{3} z_{7}^{2}-z_{7}^{4}$

$+2 z_{3} z_{6}+z_{6} z_{7}^{2}-z_{5} z_{7}-z_{1} z_{7}^{2}+z_{4}+z_{3}+z_{7}^{2}$

$\chi_{0002010}=z_{4}^{2} z_{6}-z_{2} z_{3} z_{5} z_{6}-z_{2} z_{3} z_{4} z_{7}+z_{3}^{2} z_{5} z_{7}+z_{2}^{2} z_{3}^{2}+z_{1} z_{4} z_{6}^{2}+z_{1} z_{2}^{2} z_{5} z_{7}-z_{1} z_{4} z_{5} z_{7}-z_{3}^{2} z_{4}$ 
- $z_{2} z_{4} z_{6} z_{7}-z_{1} z_{2}^{2} z_{4}+2 z_{1} z_{4}^{2}+z_{3} z_{6}^{3}-z_{1} z_{2} z_{3} z_{5}-z_{3} z_{5} z_{6} z_{7}-z_{1} z_{3}^{2} z_{6}-z_{1} z_{2} z_{6}^{2} z_{7}-z_{1}^{2} z_{2}^{2} z_{6}$

$+3 z_{1}^{2} z_{4} z_{6}+z_{2}^{2} z_{3} z_{6}+z_{5}^{2} z_{7}^{2}-z_{1}^{2} z_{2} z_{3} z_{7}-z_{3} z_{4} z_{6}+z_{2} z_{3}^{2} z_{7}-z_{5}^{2} z_{6}+z_{1} z_{2} z_{4} z_{7}-z_{1} z_{3} z_{6} z_{7}^{2}+z_{1}^{3} z_{6}^{2}$

$-z_{4} z_{6} z_{7}^{2}+z_{2} z_{3} z_{7}^{3}+2 z_{4} z_{6}^{2}-z_{1}^{3} z_{5} z_{7}+3 z_{1} z_{3} z_{5} z_{7}-4 z_{4} z_{5} z_{7}+z_{2} z_{6} z_{7}^{3}+z_{1}^{2} z_{2} z_{6} z_{7}+2 z_{1}^{3} z_{4}$

$-z_{2} z_{3} z_{6} z_{7}+z_{1} z_{2}^{2} z_{7}^{2}+z_{1} z_{6}^{3}-4 z_{1} z_{3} z_{4}-z_{1}^{2} z_{2} z_{5}+z_{1}^{2} z_{3} z_{7}^{2}-2 z_{1} z_{5} z_{6} z_{7}+2 z_{1}^{4} z_{6}-z_{2} z_{6}^{2} z_{7}+2 z_{4}^{2}$

$-2 z_{1} z_{4} z_{7}^{2}+2 z_{2} z_{3} z_{5}-z_{2} z_{5} z_{7}^{2}-z_{1}^{2} z_{6} z_{7}^{2}-z_{1} z_{2}^{2} z_{6}-z_{1} z_{5}^{2}-5 z_{1}^{2} z_{3} z_{6}-z_{3} z_{6} z_{7}^{2}+z_{3}^{2} z_{6}+4 z_{1} z_{4} z_{6}$

$-z_{1} z_{2} z_{3} z_{7}+z_{1}^{2} z_{6}^{2}-z_{6}^{2} z_{7}^{2}+2 z_{2} z_{5} z_{6}+z_{3} z_{6}^{2}-z_{1} z_{2} z_{7}^{3}-z_{2} z_{4} z_{7}+2 z_{5} z_{7}^{3}-z_{1}^{2} z_{5} z_{7}+z_{6}^{3}-2 z_{1}^{3} z_{7}^{2}$

$+3 z_{3} z_{5} z_{7}+z_{2}^{2} z_{3}-2 z_{1}^{2} z_{4}+3 z_{1} z_{3} z_{7}^{2}+z_{1} z_{2} z_{5}-2 z_{5} z_{6} z_{7}-3 z_{4} z_{7}^{2}-2 z_{1} z_{3} z_{6}+5 z_{4} z_{6}-z_{1} z_{6} z_{7}^{2}$

$+2 z_{1} z_{6}^{2}+z_{2} z_{3} z_{7}+z_{2} z_{7}^{3}+z_{7}^{4}+z_{1} z_{5} z_{7}-z_{3}^{2}-z_{1} z_{2}^{2}+2 z_{1} z_{4}-2 z_{2} z_{6} z_{7}+2 z_{2} z_{5}+2 z_{1}^{2} z_{7}^{2}-z_{1}^{2} z_{6}$

$+2 z_{1}^{3}+z_{1} z_{2} z_{7}-3 z_{6} z_{7}^{2}+3 z_{6}^{2}-z_{5} z_{7}-4 z_{1} z_{3}+3 z_{4}+z_{1} z_{6}-z_{2} z_{7}-2 z_{1}^{2}-2 z_{7}^{2}+3 z_{6}+1$

$\chi_{1000110}=z_{1} z_{5} z_{6}-z_{2} z_{6}^{2}-z_{1} z_{4} z_{7}-z_{1}^{2} z_{6} z_{7}+z_{3} z_{6} z_{7}+z_{1} z_{2} z_{7}^{2}+z_{2} z_{4}+z_{6}^{2} z_{7}+z_{1}^{2} z_{5}-z_{5} z_{7}^{2}-z_{3} z_{5}$

$-z_{5} z_{6}+z_{4} z_{7}+z_{1} z_{6} z_{7}-z_{2} z_{3}-z_{2} z_{7}^{2}-z_{2} z_{6}-z_{1} z_{2}-z_{7}^{3}+2 z_{6} z_{7}+z_{1} z_{7}+z_{7}$

$\chi_{0100110}=z_{2} z_{5} z_{6}-z_{3} z_{6}^{2}-z_{2} z_{4} z_{7}-z_{6}^{3}+z_{3} z_{4}+z_{1} z_{3} z_{7}^{2}+z_{5} z_{6} z_{7}-z_{4} z_{7}^{2}+z_{1} z_{6} z_{7}^{2}+z_{4} z_{6}-z_{1} z_{6}^{2}$

$-z_{1}^{2} z_{2} z_{7}+z_{2} z_{3} z_{7}-z_{1} z_{5} z_{7}-2 z_{1}^{2} z_{7}^{2}-z_{3}^{2}+2 z_{1} z_{4}+2 z_{1}^{2} z_{6}+z_{2} z_{5}+z_{3} z_{7}^{2}-2 z_{3} z_{6}+2 z_{1} z_{2} z_{7}$

$+z_{6} z_{7}^{2}-2 z_{6}^{2}+2 z_{1}^{3}-4 z_{1} z_{3}+2 z_{1} z_{7}^{2}+z_{4}-3 z_{1} z_{6}-z_{1}^{2}-z_{3}-z_{6}-2 z_{1}$

$\chi_{0010110}=z_{3} z_{5} z_{6}-z_{1} z_{2} z_{6}^{2}-z_{3} z_{4} z_{7}+z_{2}^{2} z_{6} z_{7}+z_{1} z_{2} z_{4}+z_{1}^{2} z_{2} z_{7}^{2}+z_{1} z_{6}^{2} z_{7}-z_{2} z_{3} z_{7}^{2}-z_{2}^{2} z_{5}+z_{4} z_{5}$

$-z_{1} z_{5} z_{7}^{2}-z_{1}^{2} z_{7}^{3}-z_{1}^{2} z_{3} z_{7}+z_{3}^{2} z_{7}-z_{1} z_{2}^{2} z_{7}+z_{1} z_{4} z_{7}+z_{1}^{2} z_{6} z_{7}-z_{6} z_{7}^{3}-z_{2} z_{6}^{2}+z_{2} z_{5} z_{7}+z_{3} z_{6} z_{7}$

$-z_{1} z_{2} z_{7}^{2}+2 z_{6}^{2} z_{7}+z_{1}^{2} z_{5}+z_{5} z_{7}^{2}+z_{1} z_{7}^{3}-z_{3} z_{5}-z_{1} z_{2} z_{6}+z_{2}^{2} z_{7}+z_{1}^{3} z_{7}+z_{1} z_{3} z_{7}-2 z_{5} z_{6}-z_{4} z_{7}$

$-z_{2} z_{3}-z_{1} z_{5}-z_{1}^{2} z_{7}-2 z_{2} z_{6}+z_{3} z_{7}+z_{6} z_{7}-z_{5}-z_{2}$

$\chi_{0001110}=z_{4} z_{5} z_{6}-z_{4}^{2} z_{7}-z_{2} z_{3} z_{6}^{2}+z_{3}^{2} z_{6} z_{7}+z_{2} z_{3} z_{4}-z_{3}^{2} z_{5}+z_{1} z_{2}^{2} z_{6} z_{7}-z_{1} z_{4} z_{6} z_{7}-z_{1} z_{2}^{2} z_{5}+2 z_{1} z_{4} z_{5}$

$-z_{2} z_{4} z_{7}^{2}+z_{3} z_{6}^{2} z_{7}-z_{1} z_{3}^{2} z_{7}-z_{1}^{2} z_{2}^{2} z_{7}+z_{1}^{2} z_{5} z_{6}-2 z_{3} z_{5} z_{6}-2 z_{1} z_{2} z_{6} z_{7}^{2}+z_{5} z_{6} z_{7}^{2}-z_{5} z_{6}^{2}+z_{2}^{2} z_{3} z_{7}$

$+2 z_{3} z_{4} z_{7}+z_{1}^{2} z_{2} z_{3}+2 z_{1} z_{2} z_{5} z_{7}-z_{1} z_{3} z_{7}^{3}-z_{5}^{2} z_{7}-z_{2} z_{3}^{2}+z_{4} z_{7}^{3}-z_{1}^{3} z_{6} z_{7}+4 z_{1} z_{3} z_{6} z_{7}+z_{1}^{2} z_{2} z_{7}^{2}$

$-2 z_{4} z_{6} z_{7}+2 z_{1}^{3} z_{5}+z_{2} z_{7}^{4}-5 z_{1} z_{3} z_{5}+z_{1} z_{6}^{2} z_{7}+3 z_{4} z_{5}+z_{2} z_{3} z_{7}^{2}-z_{2} z_{3} z_{6}-z_{1} z_{5} z_{7}^{2}-z_{2} z_{6} z_{7}^{2}$

$+z_{1}^{4} z_{7}+z_{1}^{2} z_{7}^{3}-2 z_{1} z_{5} z_{6}-z_{1}^{2} z_{3} z_{7}-z_{3}^{2} z_{7}+2 z_{1} z_{4} z_{7}-z_{3} z_{7}^{3}+z_{2} z_{5} z_{7}-2 z_{1} z_{2} z_{3}+z_{3} z_{6} z_{7}+z_{2} z_{4}$

$-z_{1} z_{2} z_{7}^{2}-z_{1}^{2} z_{5}-2 z_{1}^{3} z_{7}-3 z_{3} z_{5}+z_{1} z_{2} z_{6}-z_{5} z_{7}^{2}+z_{2}^{2} z_{7}-z_{5} z_{6}-z_{4} z_{7}-3 z_{1} z_{7}^{3}+4 z_{1} z_{6} z_{7}$

$+z_{1}^{2} z_{2}-z_{2} z_{3}-3 z_{1} z_{5}-z_{2} z_{7}^{2}+z_{3} z_{7}+z_{6} z_{7}-z_{1} z_{2}+3 z_{1} z_{7}$

$\chi_{0000210}$

$=z_{5}^{2} z_{6}-z_{4} z_{6}^{2}-z_{4} z_{5} z_{7}+z_{2} z_{3} z_{6} z_{7}-z_{1} z_{6}^{3}+z_{4}^{2}+z_{2} z_{6}^{2} z_{7}-z_{2} z_{3} z_{5}+z_{1} z_{5}^{2}+z_{1} z_{4} z_{6}$

$-z_{2} z_{5} z_{7}^{2}-z_{2} z_{5} z_{6}-z_{1} z_{2} z_{3} z_{7}+z_{2} z_{4} z_{7}+z_{3} z_{6} z_{7}^{2}-z_{1} z_{2} z_{7}^{3}+z_{6}^{2} z_{7}^{2}-z_{3} z_{6}^{2}+z_{3} z_{5} z_{7}-z_{6}^{3}+z_{1}^{2} z_{4}$

$+z_{1} z_{2} z_{6} z_{7}+z_{5} z_{6} z_{7}-z_{3} z_{4}+z_{1}^{3} z_{6}-2 z_{1} z_{3} z_{6}-z_{4} z_{6}+z_{1}^{2} z_{2} z_{7}-2 z_{1} z_{6}^{2}+2 z_{1} z_{5} z_{7}+z_{1}^{2} z_{7}^{2}+z_{1}^{2} z_{3}$

$-z_{3}^{2}-3 z_{1} z_{4}+z_{2} z_{6} z_{7}+z_{6} z_{7}^{2}-2 z_{1}^{2} z_{6}-3 z_{3} z_{6}-3 z_{6}^{2}-z_{1}^{3}-2 z_{1} z_{6}+z_{1}^{2}-z_{3}-2 z_{6}-z_{1}$

$\chi_{1000020}=z_{1} z_{6}^{2}-z_{1} z_{5} z_{7}-z_{2} z_{6} z_{7}-z_{1}^{2} z_{7}^{2}+z_{3} z_{7}^{2}+z_{2} z_{5}+z_{1}^{2} z_{6}-z_{3} z_{6}+z_{1} z_{2} z_{7}+z_{1} z_{7}^{2}-z_{2} z_{7}$

$\chi_{0100020}=z_{2} z_{6}^{2}-z_{2} z_{5} z_{7}-z_{3} z_{6} z_{7}-z_{6}^{2} z_{7}+z_{3} z_{5}+z_{1} z_{3} z_{7}+2 z_{5} z_{6}+z_{1} z_{7}^{3}-z_{4} z_{7}-2 z_{1} z_{6} z_{7}$

$-z_{1}^{2} z_{2}+z_{2} z_{3}-z_{2} z_{7}^{2}+2 z_{1} z_{5}-z_{1}^{2} z_{7}+2 z_{2} z_{6}+z_{3} z_{7}+z_{1} z_{2}+z_{5}+z_{1} z_{7}+z_{2}$

$\chi_{0010020}=z_{3} z_{6}^{2}-z_{3} z_{5} z_{7}-z_{1} z_{2} z_{6} z_{7}+z_{2}^{2} z_{7}^{2}+z_{1} z_{2} z_{5}-z_{4} z_{7}^{2}-z_{2}^{2} z_{6}+2 z_{4} z_{6}+z_{1}^{2} z_{2} z_{7}-z_{1}^{2} z_{3}+z_{1} z_{6}^{2}$

$-z_{2} z_{3} z_{7}-z_{1} z_{2}^{2}-z_{1} z_{5} z_{7}-z_{1}^{2} z_{7}^{2}+z_{3}^{2}+2 z_{1} z_{4}+2 z_{1}^{2} z_{6}-z_{1} z_{2} z_{7}-z_{2}^{2}+z_{1}^{3}+z_{4}$

$\chi_{0001020}=z_{4} z_{6}^{2}-z_{4} z_{5} z_{7}-z_{2} z_{3} z_{6} z_{7}+z_{3}^{2} z_{7}^{2}+z_{2} z_{3} z_{5}+z_{1} z_{2}^{2} z_{7}^{2}-z_{3}^{2} z_{6}-2 z_{1} z_{4} z_{7}^{2}-z_{1} z_{2}^{2} z_{6}+3 z_{1} z_{4} z_{6}$

$-z_{2} z_{4} z_{7}-z_{1} z_{2} z_{7}^{3}+z_{1}^{2} z_{6}^{2}+z_{5} z_{7}^{3}-z_{1} z_{3}^{2}-z_{1}^{2} z_{5} z_{7}+z_{3} z_{5} z_{7}-z_{1}^{2} z_{2}^{2}+2 z_{1}^{2} z_{4}+z_{2}^{2} z_{3}-2 z_{5} z_{6} z_{7}$

$-2 z_{1}^{3} z_{7}^{2}+3 z_{1} z_{3} z_{7}^{2}+3 z_{1}^{3} z_{6}+z_{5}^{2}-z_{4} z_{7}^{2}-4 z_{1} z_{3} z_{6}+3 z_{4} z_{6}+z_{1}^{2} z_{2} z_{7}+z_{2} z_{7}^{3}+2 z_{1}^{2} z_{7}^{2}+2 z_{1}^{4}$

$-z_{1} z_{2}^{2}-4 z_{1}^{2} z_{3}+3 z_{1} z_{4}-z_{2} z_{6} z_{7}+z_{3} z_{7}^{2}+z_{7}^{4}+z_{2} z_{5}-3 z_{6} z_{7}^{2}-z_{1}^{2} z_{6}-2 z_{3} z_{6}+z_{6}^{2}-z_{1}^{3}$

$-2 z_{1} z_{3}+z_{2}^{2}-z_{1} z_{7}^{2}-z_{1} z_{6}-2 z_{1}^{2}-z_{2} z_{7}-z_{7}^{2}+2 z_{6}+2 z_{1}$ 


$$
\begin{aligned}
& \chi_{0000120}=z_{5} z_{6}^{2}-z_{5}^{2} z_{7}-z_{4} z_{6} z_{7}+z_{2} z_{3} z_{7}^{2}+z_{4} z_{5}-z_{1} z_{6}^{2} z_{7}-z_{2} z_{3} z_{6}-z_{1} z_{5} z_{7}^{2}+z_{2} z_{6} z_{7}^{2}+3 z_{1} z_{5} z_{6} \\
& -z_{2} z_{6}^{2}-z_{2} z_{5} z_{7}-z_{1} z_{2} z_{3}+z_{6} z_{7}^{3}+z_{3} z_{6} z_{7}-z_{6}^{2} z_{7}+z_{2} z_{4}+z_{1}^{2} z_{5}-z_{1} z_{2} z_{7}^{2}-z_{5} z_{7}^{2}+2 z_{5} z_{6} \\
& -z_{1} z_{7}^{3}+z_{4} z_{7}+z_{1}^{2} z_{2}-2 z_{2} z_{3}+2 z_{1} z_{6} z_{7}+z_{2} z_{7}^{2}+z_{1} z_{5}-2 z_{2} z_{6}+2 z_{1}^{2} z_{7}-z_{3} z_{7}-z_{1} z_{2}-z_{6} z_{7} \\
& -z_{1} z_{7}-z_{2} \\
& \chi_{0000030}=z_{6}^{3}-2 z_{5} z_{6} z_{7}+z_{4} z_{7}^{2}+z_{5}^{2}-z_{1} z_{6} z_{7}^{2}-z_{4} z_{6}+z_{2} z_{7}^{3}+z_{1} z_{6}^{2}+z_{1} z_{5} z_{7}-2 z_{2} z_{6} z_{7}+z_{1}^{2} z_{7}^{2} \\
& -z_{3} z_{7}^{2}-z_{1} z_{4}+z_{2} z_{5}-z_{1}^{2} z_{6}+2 z_{3} z_{6}-z_{6} z_{7}^{2}-z_{1} z_{2} z_{7}+2 z_{6}^{2}-z_{1} z_{7}^{2}-z_{1}^{3}+2 z_{1} z_{3}-z_{4}+3 z_{1} z_{6} \\
& -z_{2} z_{7}+z_{1}^{2}+z_{3}+z_{6}+z_{1} \\
& \chi_{2000001}=z_{1}^{2} z_{7}-z_{3} z_{7}-z_{1} z_{2}-z_{6} z_{7}+z_{5}-z_{1} z_{7}+z_{2} \\
& \chi_{1100001}=z_{1} z_{2} z_{7}-z_{2}^{2}-z_{1} z_{3}-z_{5} z_{7}+z_{4}-z_{1} z_{7}^{2}+z_{1} z_{6}+z_{1}^{2}-z_{3} \\
& \chi_{0200001}=z_{2}^{2} z_{7}-z_{4} z_{7}-z_{2} z_{3}-z_{1} z_{6} z_{7}+z_{1} z_{5}-z_{2} z_{6}+z_{3} z_{7}-z_{1} z_{2}+z_{6} z_{7}+z_{1} z_{7}-z_{2} \\
& \chi_{1010001}=z_{1} z_{3} z_{7}-z_{4} z_{7}-z_{1}^{2} z_{2}-z_{1} z_{6} z_{7}+z_{7}^{3}+z_{1} z_{5}+z_{2} z_{6}-z_{6} z_{7}+z_{1} z_{2}+z_{5}+z_{2}-z_{7} \\
& \chi_{0110001}=z_{2} z_{3} z_{7}-z_{1} z_{5} z_{7}-z_{3}^{2}-z_{1}^{2} z_{7}^{2}-z_{1} z_{2}^{2}+z_{3} z_{7}^{2}+z_{1} z_{4}+z_{2} z_{5}+2 z_{1}^{2} z_{6}+z_{6} z_{7}^{2}-2 z_{3} z_{6}+z_{1} z_{2} z_{7} \\
& -z_{6}^{2}+2 z_{1}^{3}-4 z_{1} z_{3}+z_{4}-2 z_{1} z_{6}-z_{1}^{2}+z_{2} z_{7}-2 z_{3}-2 z_{6}-2 z_{1} \\
& \chi_{0020001}=z_{3}^{2} z_{7}-z_{1} z_{4} z_{7}-z_{1} z_{2} z_{3}-z_{1}^{2} z_{6} z_{7}+z_{3} z_{6} z_{7}+z_{2} z_{4}+z_{1}^{2} z_{5}-z_{3} z_{5}+z_{5} z_{7}^{2}+z_{1} z_{2} z_{6}-z_{5} z_{6} \\
& +z_{1} z_{7}^{3}+z_{1}^{2} z_{2}-z_{2} z_{3}-z_{1} z_{6} z_{7}-z_{1} z_{5}-z_{2} z_{7}^{2}-z_{1}^{2} z_{7}+z_{2} z_{6}+z_{3} z_{7}-z_{5} \\
& \chi_{1001001}=z_{1} z_{4} z_{7}-z_{2} z_{5} z_{7}-z_{1} z_{2} z_{3}+z_{6}^{2} z_{7}+z_{3} z_{5}-z_{5} z_{7}^{2}+z_{1} z_{2} z_{6}+z_{1}^{3} z_{7}-z_{1} z_{3} z_{7}-z_{1} z_{6} z_{7}-z_{2} z_{7}^{2} \\
& +z_{1} z_{5}-2 z_{1}^{2} z_{7}+z_{3} z_{7}+z_{1} z_{2}-z_{7}^{3}+2 z_{6} z_{7}+z_{1} z_{7}+z_{7} \\
& \chi_{0101001}=z_{2} z_{4} z_{7}-z_{3} z_{5} z_{7}-z_{2}^{2} z_{3}+z_{1} z_{2} z_{5}+z_{1} z_{3} z_{6}+z_{1} z_{6} z_{7}^{2}-z_{2} z_{7}^{3}-z_{4} z_{6}+z_{1}^{2} z_{2} z_{7}-z_{1} z_{6}^{2}-z_{2} z_{3} z_{7} \\
& -z_{1} z_{5} z_{7}-z_{1}^{2} z_{7}^{2}+z_{2} z_{6} z_{7}-z_{1}^{2} z_{6}-z_{2} z_{5}+z_{3} z_{6}+z_{1} z_{2} z_{7}-z_{2}^{2}+z_{1} z_{7}^{2} \\
& \chi_{0011001}=z_{3} z_{4} z_{7}-z_{1} z_{2} z_{5} z_{7}-z_{2} z_{3}^{2}+z_{1} z_{3} z_{5}+z_{4} z_{6} z_{7}+z_{1} z_{6}^{2} z_{7}+z_{2}^{2} z_{5}-z_{4} z_{5}+z_{1}^{2} z_{2} z_{6}-z_{2} z_{3} z_{6} \\
& +z_{1}^{2} z_{3} z_{7}-z_{3}^{2} z_{7}-z_{1} z_{5} z_{6}-z_{1} z_{4} z_{7}-z_{1}^{2} z_{6} z_{7}-z_{2} z_{6}^{2}-z_{6} z_{7}^{3}+z_{2} z_{5} z_{7}-z_{3} z_{6} z_{7}+z_{6}^{2} z_{7}-z_{1} z_{2} z_{7}^{2} \\
& +\quad z_{5} z_{7}^{2}-z_{5} z_{6}-z_{1}^{3} z_{7}+z_{1} z_{3} z_{7}+z_{1} z_{7}^{3}+z_{2}^{2} z_{7}+z_{1}^{2} z_{2}+z_{1} z_{6} z_{7}-z_{2} z_{3}-z_{1} z_{5} \\
& +z_{1}^{2} z_{7}-2 z_{2} z_{6}+z_{6} z_{7}-z_{1} z_{2}-z_{5}-z_{1} z_{7} \\
& \chi_{0002001}=z_{4}^{2} z_{7}-z_{2} z_{3} z_{5} z_{7}-z_{2} z_{3} z_{4}+z_{1} z_{4} z_{6} z_{7}+z_{3}^{2} z_{5}+z_{1} z_{2}^{2} z_{5}-z_{1} z_{4} z_{5}+z_{3} z_{6}^{2} z_{7}-z_{2} z_{4} z_{6}+z_{1}^{2} z_{5} z_{7}^{2} \\
& -2 z_{3} z_{5} z_{7}^{2}-z_{1}^{2} z_{5} z_{6}+z_{3} z_{5} z_{6}-z_{1} z_{2} z_{6}^{2}-2 z_{4} z_{7}^{3}-z_{1} z_{6} z_{7}^{3}-z_{1} z_{3} z_{6} z_{7}+3 z_{4} z_{6} z_{7}-2 z_{1}^{3} z_{5} \\
& +5 z_{1} z_{3} z_{5}-3 z_{4} z_{5}+2 z_{1} z_{6}^{2} z_{7}+z_{2} z_{3} z_{7}^{2}-z_{1} z_{5} z_{7}^{2}-z_{2} z_{3} z_{6}+z_{2} z_{6} z_{7}^{2}-z_{2} z_{6}^{2}+2 z_{1} z_{4} z_{7}+2 z_{1}^{2} z_{6} z_{7} \\
& +z_{7}^{5}-z_{3} z_{6} z_{7}-3 z_{6} z_{7}^{3}+2 z_{6}^{2} z_{7}+z_{5} z_{7}^{2}-2 z_{2} z_{4}+3 z_{3} z_{5}-2 z_{1} z_{2} z_{6}-2 z_{1} z_{7}^{3}-z_{5} z_{6}+2 z_{4} z_{7} \\
& +3 z_{1} z_{6} z_{7}+2 z_{2} z_{7}^{2}-z_{2} z_{3}+z_{1} z_{5}-2 z_{7}^{3}-3 z_{2} z_{6}+3 z_{6} z_{7}-z_{5}+2 z_{1} z_{7}-2 z_{2}+z_{7} \\
& \chi_{1000101}=z_{1} z_{5} z_{7}-z_{2} z_{6} z_{7}-z_{1} z_{4}-z_{1}^{2} z_{6}+z_{3} z_{6}+z_{6}^{2}+z_{1} z_{2} z_{7}-z_{5} z_{7}+z_{1} z_{7}^{2}-z_{2} z_{7}-z_{7}^{2} \\
& -z_{1}^{2}+z_{3}+2 z_{6}+1 \\
& \chi_{0100101}=z_{2} z_{5} z_{7}-z_{3} z_{6} z_{7}-z_{6}^{2} z_{7}-z_{2} z_{4}+z_{5} z_{7}^{2}+z_{1} z_{3} z_{7}+z_{1} z_{7}^{3}-z_{4} z_{7}-z_{1} z_{6} z_{7}-z_{1} z_{5} \\
& -2 z_{1}^{2} z_{7}+z_{3} z_{7}+z_{1} z_{2}-z_{5}+z_{7} \\
& \chi_{0010101}=z_{3} z_{5} z_{7}-z_{1} z_{2} z_{6} z_{7}-z_{3} z_{4}+z_{4} z_{7}^{2}+z_{1} z_{6} z_{7}^{2}+z_{2}^{2} z_{6}-z_{4} z_{6}+z_{2} z_{7}^{3}+z_{1}^{2} z_{2} z_{7}-z_{7}^{4}-z_{2} z_{3} z_{7} \\
& -z_{1} z_{5} z_{7}-2 z_{2} z_{6} z_{7}-z_{1} z_{4}-z_{1}^{2} z_{6}+z_{2} z_{5}-z_{3} z_{7}^{2}+z_{3} z_{6}-2 z_{1} z_{2} z_{7}-z_{1}^{3}+z_{2}^{2}+z_{6} z_{7}^{2}+z_{6}^{2} \\
& -z_{5} z_{7}+2 z_{1} z_{3}+2 z_{1} z_{6}+z_{1}^{2}-2 z_{2} z_{7}+z_{7}^{2}+z_{3}+z_{6}+z_{1} \\
& \chi_{0001101}=z_{4} z_{5} z_{7}-z_{2} z_{3} z_{6} z_{7}-z_{4}^{2}+z_{1} z_{4} z_{7}^{2}+z_{3}^{2} z_{6}+z_{1} z_{2}^{2} z_{6}-2 z_{1} z_{4} z_{6}+z_{1}^{2} z_{6} z_{7}^{2}-z_{2} z_{4} z_{7}-z_{1}^{2} z_{6}^{2}+z_{3} z_{6}^{2} \\
& -z_{3} z_{5} z_{7}-2 z_{1} z_{2} z_{6} z_{7}-z_{1}^{2} z_{4}+z_{3} z_{4}+z_{1} z_{2} z_{5}-z_{1} z_{7}^{4}-z_{1} z_{3} z_{7}^{2}-2 z_{1}^{3} z_{6}+4 z_{1} z_{3} z_{6}-z_{4} z_{6} \\
& +z_{1} z_{6} z_{7}^{2}+z_{2} z_{7}^{3}+z_{2} z_{3} z_{7}+z_{1} z_{6}^{2}-z_{1} z_{5} z_{7}+z_{7}^{4}-z_{2} z_{6} z_{7}+2 z_{1}^{2} z_{7}^{2}+2 z_{1} z_{4}-z_{3} z_{7}^{2}-2 z_{6} z_{7}^{2} \\
& +\quad z_{1}^{2} z_{6}+2 z_{3} z_{6}-z_{1} z_{2} z_{7}+z_{6}^{2}-2 z_{1} z_{7}^{2}+3 z_{1} z_{6}-z_{1}^{2}+z_{3}-2 z_{7}^{2}+2 z_{6}+2 z_{1}+1
\end{aligned}
$$




$$
\begin{aligned}
& \chi_{0000201}=z_{5}^{2} z_{7}-z_{4} z_{6} z_{7}-z_{4} z_{5}-z_{1} z_{6}^{2} z_{7}+z_{2} z_{3} z_{6}+z_{1} z_{5} z_{7}^{2}-z_{1} z_{5} z_{6}+z_{2} z_{6}^{2}-z_{2} z_{5} z_{7}+z_{3} z_{7}^{3}+z_{6} z_{7}^{3} \\
& -z_{3} z_{6} z_{7}-z_{6}^{2} z_{7}-z_{1}^{2} z_{5}-z_{1} z_{2} z_{7}^{2}+z_{3} z_{5}+z_{1} z_{2} z_{6}+z_{5} z_{6}-z_{1} z_{3} z_{7}+z_{2} z_{3}-z_{1} z_{6} z_{7}+z_{1} z_{5} \\
& +2 z_{2} z_{6}+z_{1}^{2} z_{7}-2 z_{3} z_{7}+z_{7}^{3}+z_{1} z_{2}-3 z_{6} z_{7}+z_{5}-z_{1} z_{7}+z_{2}-2 z_{7} \\
& \chi_{1000011}=z_{1} z_{6} z_{7}-z_{2} z_{7}^{2}-z_{7}^{3}-z_{1} z_{5}-z_{1}^{2} z_{7}+z_{3} z_{7}+z_{1} z_{2}+2 z_{6} z_{7}-z_{5}+2 z_{1} z_{7}+z_{7} \\
& \chi_{0100011}=z_{2} z_{6} z_{7}-z_{3} z_{7}^{2}-z_{2} z_{5}-z_{6} z_{7}^{2}+z_{1} z_{3}+z_{5} z_{7}-z_{4}+z_{1} z_{7}^{2}-z_{1} z_{6}-z_{1}^{2}+z_{3} \\
& \chi_{0010011}=z_{3} z_{6} z_{7}-z_{1} z_{2} z_{7}^{2}-z_{3} z_{5}+z_{2}^{2} z_{7}+z_{1} z_{6} z_{7}+z_{1}^{2} z_{2}-z_{2} z_{3}+z_{2} z_{7}^{2}-z_{1} z_{5}-z_{2} z_{6}-z_{3} z_{7}-z_{1} z_{2} \\
& -z_{1} z_{7}-z_{2} \\
& \chi_{0001011}=z_{4} z_{6} z_{7}-z_{2} z_{3} z_{7}^{2}-z_{4} z_{5}+z_{3}^{2} z_{7}+z_{1}^{2} z_{7}^{3}+z_{1} z_{2}^{2} z_{7}-z_{1} z_{4} z_{7}-z_{3} z_{7}^{3}-z_{1}^{2} z_{6} z_{7}+2 z_{3} z_{6} z_{7}-z_{2} z_{4} \\
& -z_{1} z_{2} z_{7}^{2}-z_{1} z_{2} z_{6}-2 z_{1}^{3} z_{7}-2 z_{1} z_{7}^{3}+3 z_{1} z_{3} z_{7}+3 z_{1} z_{6} z_{7}+z_{2} z_{7}^{2}-z_{2} z_{6}+3 z_{1}^{2} z_{7}+z_{3} z_{7}-z_{1} z_{2} \\
& +2 z_{1} z_{7}-z_{2} \\
& \chi_{0000111}=z_{5} z_{6} z_{7}-z_{4} z_{7}^{2}-z_{5}^{2}-z_{1} z_{6} z_{7}^{2}+z_{2} z_{3} z_{7}+z_{2} z_{6} z_{7}+z_{7}^{4}+z_{3} z_{7}^{2}-z_{2} z_{5}-z_{6} z_{7}^{2}-z_{1} z_{2} z_{7}+z_{5} z_{7} \\
& -z_{1} z_{7}^{2}-z_{1} z_{3}+z_{4}+z_{1} z_{6}+z_{2} z_{7}-z_{7}^{2}+z_{1}^{2}-z_{3} \\
& \chi_{0000021}=z_{6}^{2} z_{7}-z_{5} z_{7}^{2}-z_{5} z_{6}+z_{4} z_{7}-z_{1} z_{7}^{3}+z_{1} z_{6} z_{7}+z_{2} z_{7}^{2}-z_{2} z_{6}+z_{1}^{2} z_{7}-z_{1} z_{2}-z_{2} \\
& \chi_{1000002}=z_{1} z_{7}^{2}-z_{1} z_{6}-z_{2} z_{7}-z_{1}^{2}-z_{7}^{2}+z_{3}+z_{6}+z_{1}+1 \\
& \chi_{0100002}=z_{2} z_{7}^{2}-z_{2} z_{6}-z_{3} z_{7}-z_{6} z_{7}-z_{2} \\
& \chi_{0010002}=z_{3} z_{7}^{2}-z_{3} z_{6}-z_{1} z_{2} z_{7}+z_{2}^{2}-z_{4}+z_{7}^{2}-1 \\
& \chi_{0001002}=z_{4} z_{7}^{2}-z_{4} z_{6}-z_{2} z_{3} z_{7}+z_{3}^{2}+z_{1}^{2} z_{7}^{2}-z_{3} z_{7}^{2}+z_{1} z_{2}^{2}-2 z_{1} z_{4}-z_{1}^{2} z_{6}+z_{3} z_{6}-z_{1} z_{2} z_{7}-z_{1} z_{7}^{2} \\
& -2 z_{1}^{3}+4 z_{1} z_{3}-2 z_{4}+z_{1} z_{6}+z_{1}^{2}+2 z_{3}+2 z_{1} \\
& \chi_{0000102}=z_{5} z_{7}^{2}-z_{5} z_{6}-z_{4} z_{7}+z_{2} z_{3}-z_{1} z_{6} z_{7}-z_{1} z_{5}+z_{7}^{3}+z_{2} z_{6}-z_{6} z_{7}-z_{1} z_{7}+z_{2}-z_{7} \\
& \chi_{0000012}=z_{6} z_{7}^{2}-z_{6}^{2}-z_{5} z_{7}+z_{4}-z_{1} z_{7}^{2}+z_{2} z_{7}+z_{1}^{2}-z_{3}-z_{6}-z_{1} \\
& \chi_{0000003}=z_{7}^{3}-2 z_{6} z_{7}+z_{5}-z_{1} z_{7}+z_{2}-z_{7}
\end{aligned}
$$

\section{Appendix B: List of the cubic Clebsch-Gordan series.}

$$
\begin{aligned}
z_{1}^{3} & =\chi_{3000000}+2 \chi_{1010000}+\chi_{0001000}+3 \chi_{1000010}+3 \chi_{2000000}+2 \chi_{0100001}+4 \chi_{0010000}+\chi_{0000002} \\
& +3 \chi_{0000010}+5 \chi_{1000000}+\chi_{0000000} \\
z_{1}^{2} z_{2} & =\chi_{2100000}+\chi_{0110000}+2 \chi_{1000100}+2 \chi_{2000001}+2 \chi_{0100010}+3 \chi_{0010001}+2 \chi_{0000011}+5 \chi_{1100000} \\
& +4 \chi_{0000100}+6 \chi_{1000001}+5 \chi_{0100000}+3 \chi_{0000001} \\
z_{1} z_{2}^{2} & =\chi_{1200000}+\chi_{1001000}+2 \chi_{0100100}+\chi_{2000010}+2 \chi_{0010010}+\chi_{0000020}+4 \chi_{1100001}+\chi_{3000000} \\
& +3 \chi_{0200000}+3 \chi_{0000101}+3 \chi_{1000002}+4 \chi_{1010000}+5 \chi_{0001000}+7 \chi_{1000010}+4 \chi_{2000000} \\
& +8 \chi_{0100001}+3 \chi_{0000002}+7 \chi_{0010000}+6 \chi_{0000010}+5 \chi_{1000000}+\chi_{0000000} \\
z_{2}^{3} & =\chi_{0300000}+2 \chi_{0101000}+\chi_{0010100}+3 \chi_{1100010}+2 \chi_{0000110}+2 \chi_{1010001}+3 \chi_{0200001}+4 \chi_{0001001} \\
& +4 \chi_{2100000}+4 \chi_{1000011}+5 \chi_{0100002}+\chi_{0000003}+6 \chi_{0110000}+8 \chi_{1000100}+6 \chi_{2000001}+9 \chi_{0100010} \\
& +12 \chi_{0010001}+12 \chi_{1100000}+8 \chi_{0000011}+11 \chi_{0000100}+12 \chi_{1000001}+10 \chi_{0100000}+4 \chi_{0000001} \\
z_{1}^{2} z_{3} & =\chi_{2010000}+\chi_{0020000}+2 \chi_{1001000}+2 \chi_{2000010}+\chi_{0100100}+3 \chi_{0010010}+\chi_{0000020}+4 \chi_{1100001} \\
& +2 \chi_{3000000}+2 \chi_{0200000}+3 \chi_{0000101}+2 \chi_{1000002}+6 \chi_{1010000}+5 \chi_{0001000}+8 \chi_{1000010} \\
& +6 \chi_{0100001}+2 \chi_{0000002}+5 \chi_{2000000}+8 \chi_{0010000}+5 \chi_{0000010}+4 \chi_{1000000}+\chi_{0000000} \\
z_{1} z_{2} z_{3} & =\chi_{1110000}+\chi_{0101000}+\chi_{2000100}+2 \chi_{0010100}+3 \chi_{1100010}+2 \chi_{0000110}+2 \chi_{0200001}+\chi_{3000001} \\
& +4 \chi_{1010001}+4 \chi_{0001001}+5 \chi_{1000011}+5 \chi_{2100000}+7 \chi_{0110000}+3 \chi_{0100002}+9 \chi_{1000100} \\
& +9 \chi_{0100010}+8 \chi_{2000001}+12 \chi_{0010001}+13 \chi_{1100000}+\chi_{0000003}+7 \chi_{0000011}+10 \chi_{0000100} \\
& +12 \chi_{1000001}+7 \chi_{0100000}+4 \chi_{0000001} \\
z_{2}^{2} z_{3} & =\chi_{0210000}+\chi_{0011000}+2 \chi_{1100100}+\chi_{0000200}+2 \chi_{1010010}+2 \chi_{0200010}+3 \chi_{0001010}+2 \chi_{1000020}
\end{aligned}
$$




$$
\begin{aligned}
& +3 \chi_{2100001}+5 \chi_{0110001}+3 \chi_{2010000}+6 \chi_{1000101}+6 \chi_{1200000}+3 \chi_{2000002}+3 \chi_{0020000} \\
& +10 \chi_{1001000}+6 \chi_{0100011}+9 \chi_{2000010}+6 \chi_{0010002}+10 \chi_{0100100}+14 \chi_{0010010}+3 \chi_{0000012} \\
& +22 \chi_{1100001}+4 \chi_{3000000}+8 \chi_{0200000}+5 \chi_{0000020}+16 \chi_{0000101}+12 \chi_{1000002}+16 \chi_{1010000} \\
& +18 \chi_{0001000}+23 \chi_{1000010}+9 \chi_{2000000}+21 \chi_{0100001}+17 \chi_{0010000}+6 \chi_{0000002}+12 \chi_{0000010} \\
& +7 \chi_{1000000}+\chi_{0000000} \\
& z_{1} z_{3}^{2}=\chi_{1020000}+\chi_{2001000}+2 \chi_{0011000}+2 \chi_{1100100}+\chi_{0000200}+\chi_{0200010}+\chi_{3000010}+4 \chi_{1010010} \\
& +4 \chi_{2100001}+3 \chi_{0001010}+\chi_{4000000}+6 \chi_{0110001}+5 \chi_{1200000}+6 \chi_{2010000}+3 \chi_{1000020}+7 \chi_{1000101} \\
& +5 \chi_{0020000}+4 \chi_{2000002}+6 \chi_{0100011}+12 \chi_{1001000}+5 \chi_{0010002}+12 \chi_{2000010}+10 \chi_{0100100} \\
& +3 \chi_{0000012}+17 \chi_{0010010}+22 \chi_{1100001}+6 \chi_{3000000}+6 \chi_{0000020}+20 \chi_{1010000}+15 \chi_{0000101} \\
& +8 \chi_{0200000}+11 \chi_{1000002}+18 \chi_{0001000}+25 \chi_{1000010}+19 \chi_{0100001}+11 \chi_{2000000}+6 \chi_{0000002} \\
& +16 \chi_{0010000}+11 \chi_{0000010}+8 \chi_{1000000}+\chi_{0000000} \\
& z_{2} z_{3}^{2}=\chi_{0120000}+\chi_{1101000}+2 \chi_{1010100}+\chi_{0200100}+2 \chi_{0001100}+2 \chi_{2100010}+4 \chi_{0110010}+4 \chi_{1200001} \\
& +3 \chi_{2010001}+4 \chi_{1000110}+3 \chi_{0020001}+8 \chi_{1001001}+6 \chi_{2000011}+3 \chi_{0100020}+3 \chi_{3100000}+7 \chi_{0100101} \\
& +10 \chi_{0010011}+2 \chi_{0300000}+12 \chi_{1110000}+12 \chi_{2000100}+11 \chi_{0101000}+10 \chi_{1100002}+16 \chi_{0010100} \\
& +28 \chi_{1100010}+3 \chi_{0000021}+8 \chi_{3000001}+7 \chi_{0000102}+4 \chi_{1000003}+16 \chi_{0000110}+30 \chi_{1010001} \\
& +14 \chi_{0200001}+24 \chi_{2100000}+30 \chi_{0001001}+32 \chi_{0110000}+32 \chi_{1000011}+20 \chi_{0100002}+42 \chi_{1000100} \\
& +4 \chi_{0000003}+29 \chi_{2000001}+38 \chi_{0100010}+46 \chi_{0010001}+39 \chi_{1100000}+25 \chi_{0000011}+30 \chi_{0000100} \\
& +30 \chi_{1000001}+17 \chi_{0100000}+7 \chi_{0000001} \\
& z_{3}^{3}=\chi_{0030000}+2 \chi_{1011000}+\chi_{0002000}+\chi_{2100100}+3 \chi_{0110100}+2 \chi_{1200010}+3 \chi_{2010010}+3 \chi_{0020010} \\
& +2 \chi_{1000200}+6 \chi_{1001010}+3 \chi_{2000020}+\chi_{0300001}+4 \chi_{0100110}+6 \chi_{0010020}+2 \chi_{3100001}+4 \chi_{3010000} \\
& +10 \chi_{1110001}+9 \chi_{2000101}+8 \chi_{0101001}+\chi_{0000030}+6 \chi_{2200000}+12 \chi_{0010101}+9 \chi_{0210000} \\
& +18 \chi_{1100011}+8 \chi_{1020000}+6 \chi_{0200002}+4 \chi_{3000002}+15 \chi_{2001000}+18 \chi_{0011000}+14 \chi_{1010002} \\
& +30 \chi_{1100100}+10 \chi_{0000111}+11 \chi_{0000200}+14 \chi_{0001002}+12 \chi_{3000010}+42 \chi_{1010010}+42 \chi_{2100001} \\
& +13 \chi_{1000012}+5 \chi_{4000000}+18 \chi_{0200010}+36 \chi_{0001010}+56 \chi_{0110001}+27 \chi_{1000020}+34 \chi_{1200000} \\
& +66 \chi_{1000101}+7 \chi_{0100003}+29 \chi_{2000002}+33 \chi_{2010000}+29 \chi_{0020000}+52 \chi_{0100011}+\chi_{0000004} \\
& +45 \chi_{0010002}+72 \chi_{1001000}+63 \chi_{2000010}+61 \chi_{0100100}+90 \chi_{0010010}+21 \chi_{0000012}+112 \chi_{1100001} \\
& +21 \chi_{3000000}+29 \chi_{0000020}+75 \chi_{0000101}+33 \chi_{0200000}+74 \chi_{1010000}+48 \chi_{1000002}+72 \chi_{0001000} \\
& +88 \chi_{1000010}+66 \chi_{0100001}+30 \chi_{2000000}+50 \chi_{0010000}+17 \chi_{0000002}+30 \chi_{0000010}+16 \chi_{1000000} \\
& +2 \chi_{0000000} \\
& z_{1}^{2} z_{4}=\chi_{2001000}+\chi_{0011000}+2 \chi_{1100100}+\chi_{0000200}+\chi_{0200010}+2 \chi_{1010010}+3 \chi_{0001010}+2 \chi_{2100001} \\
& +4 \chi_{0110001}+\chi_{1000020}+4 \chi_{1200000}+2 \chi_{2010000}+5 \chi_{1000101}+\chi_{2000002}+3 \chi_{0020000}+4 \chi_{0100011} \\
& +3 \chi_{0010002}+7 \chi_{1001000}+8 \chi_{0100100}+5 \chi_{2000010}+\chi_{0000012}+9 \chi_{0010010}+3 \chi_{0000020}+\chi_{3000000} \\
& +12 \chi_{1100001}+8 \chi_{0000101}+8 \chi_{1010000}+5 \chi_{0200000}+11 \chi_{0001000}+4 \chi_{1000002}+9 \chi_{1000010} \\
& +3 \chi_{2000000}+8 \chi_{0100001}+\chi_{0000002}+5 \chi_{0010000}+3 \chi_{0000010}+\chi_{1000000} \\
& z_{1} z_{2} z_{4}=\chi_{1101000}+\chi_{1010100}+\chi_{0200100}+2 \chi_{0001100}+\chi_{2100010}+3 \chi_{0110010}+\chi_{2010001}+3 \chi_{1200001} \\
& +2 \chi_{0020001}+3 \chi_{1000110}+5 \chi_{1001001}+\chi_{3100000}+3 \chi_{2000011}+2 \chi_{0300000}+2 \chi_{0100020}+6 \chi_{0100101} \\
& +6 \chi_{0010011}+7 \chi_{1110000}+6 \chi_{1100002}+8 \chi_{0101000}+2 \chi_{0000021}+4 \chi_{0000102}+6 \chi_{2000100} \\
& +11 \chi_{0010100}+17 \chi_{1100010}+3 \chi_{3000001}+10 \chi_{0200001}+2 \chi_{1000003}+15 \chi_{1010001}+10 \chi_{0000110} \\
& +11 \chi_{2100000}+18 \chi_{0001001}+19 \chi_{0110000}+16 \chi_{1000011}+10 \chi_{0100002}+2 \chi_{0000003}+21 \chi_{1000100} \\
& +12 \chi_{2000001}+20 \chi_{0100010}+20 \chi_{0010001}+10 \chi_{0000011}+17 \chi_{1100000}+12 \chi_{0000100}+10 \chi_{1000001} \\
& +5 \chi_{0100000}+2 \chi_{0000001} \\
& z_{2}^{2} z_{4}=\chi_{0201000}+\chi_{0002000}+2 \chi_{0110100}+\chi_{0020010}+\chi_{1000200}+2 \chi_{1200010}+3 \chi_{1001010}+4 \chi_{0100110} \\
& +\chi_{2000020}+4 \chi_{1110001}+2 \chi_{0300001}+6 \chi_{0101001}+3 \chi_{0010020}+3 \chi_{2000101}+7 \chi_{0010101}+2 \chi_{1020000} \\
& +3 \chi_{2200000}+5 \chi_{2001000}+10 \chi_{1100011}+\chi_{0000030}+5 \chi_{0200002}+7 \chi_{0210000}+6 \chi_{0000111}+\chi_{3000002} \\
& +9 \chi_{0011000}+18 \chi_{1100100}+6 \chi_{1010002}+7 \chi_{0000200}+13 \chi_{0200010}+9 \chi_{0001002}+3 \chi_{3000010} \\
& +18 \chi_{1010010}+18 \chi_{2100001}+7 \chi_{1000012}+21 \chi_{0001010}+13 \chi_{1000020}+32 \chi_{0110001}+34 \chi_{1000101}
\end{aligned}
$$




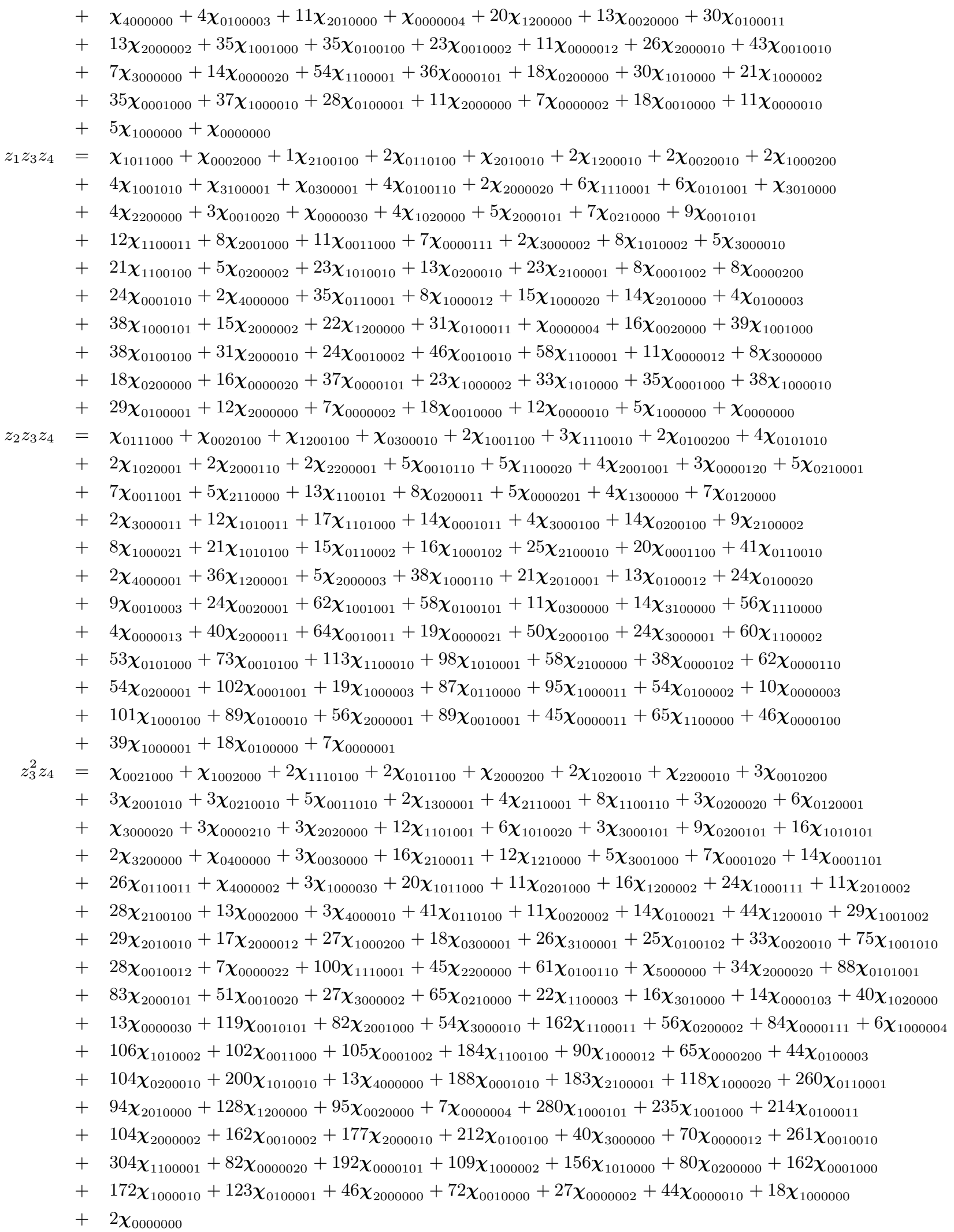

$z_{1} z_{4}^{2}=\chi_{1002000}+\chi_{1110100}+2 \chi_{0101100}+\chi_{2000200}+\chi_{1020010}+\chi_{2200010}+2 \chi_{0010200}+\chi_{2001010}$

$+2 \chi_{0210010}+4 \chi_{0011010}+2 \chi_{1300001}+6 \chi_{1100110}+3 \chi_{0200020}+\chi_{0400000}+2 \chi_{2110001}+\chi_{3000020}$ 


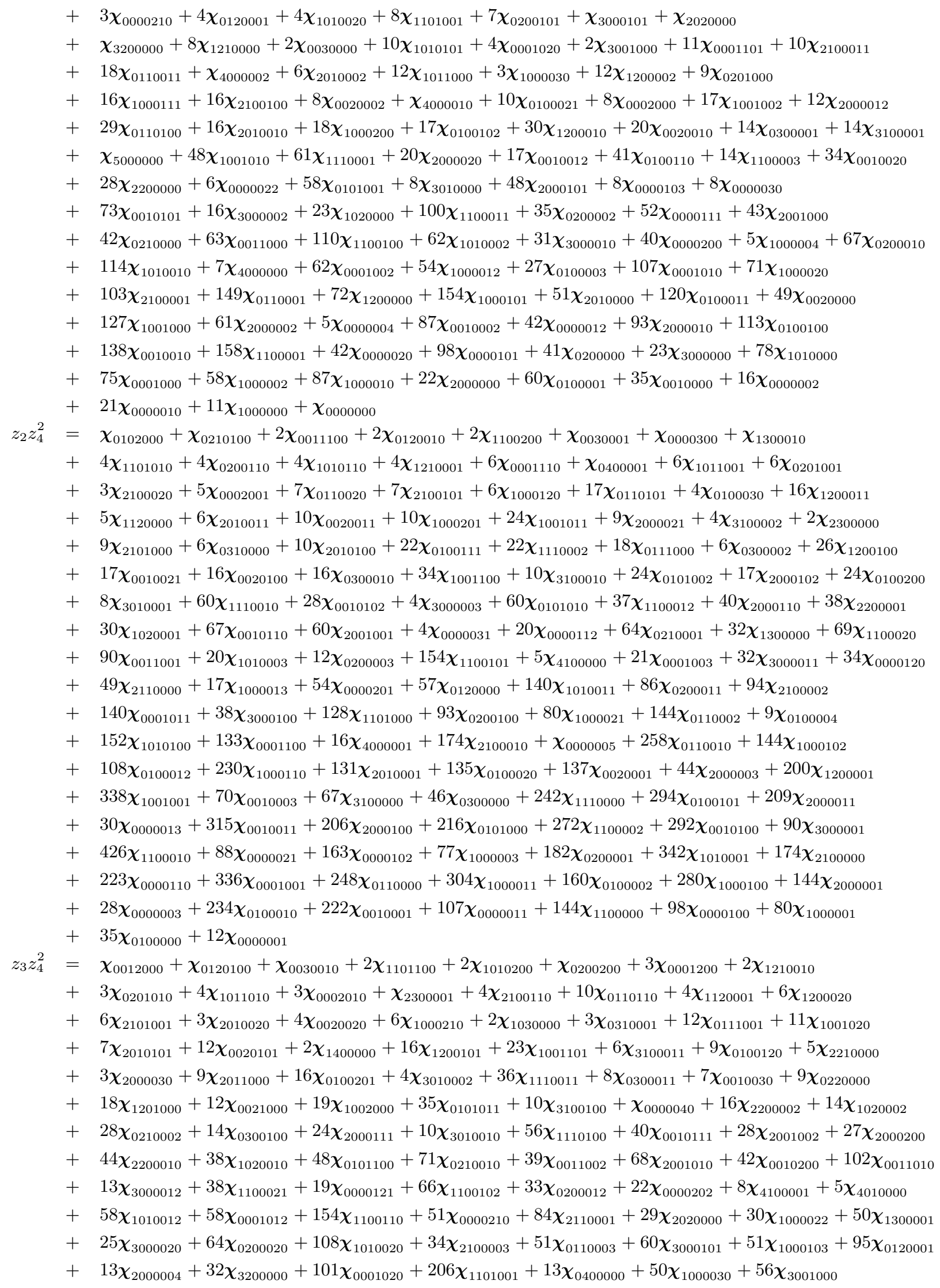




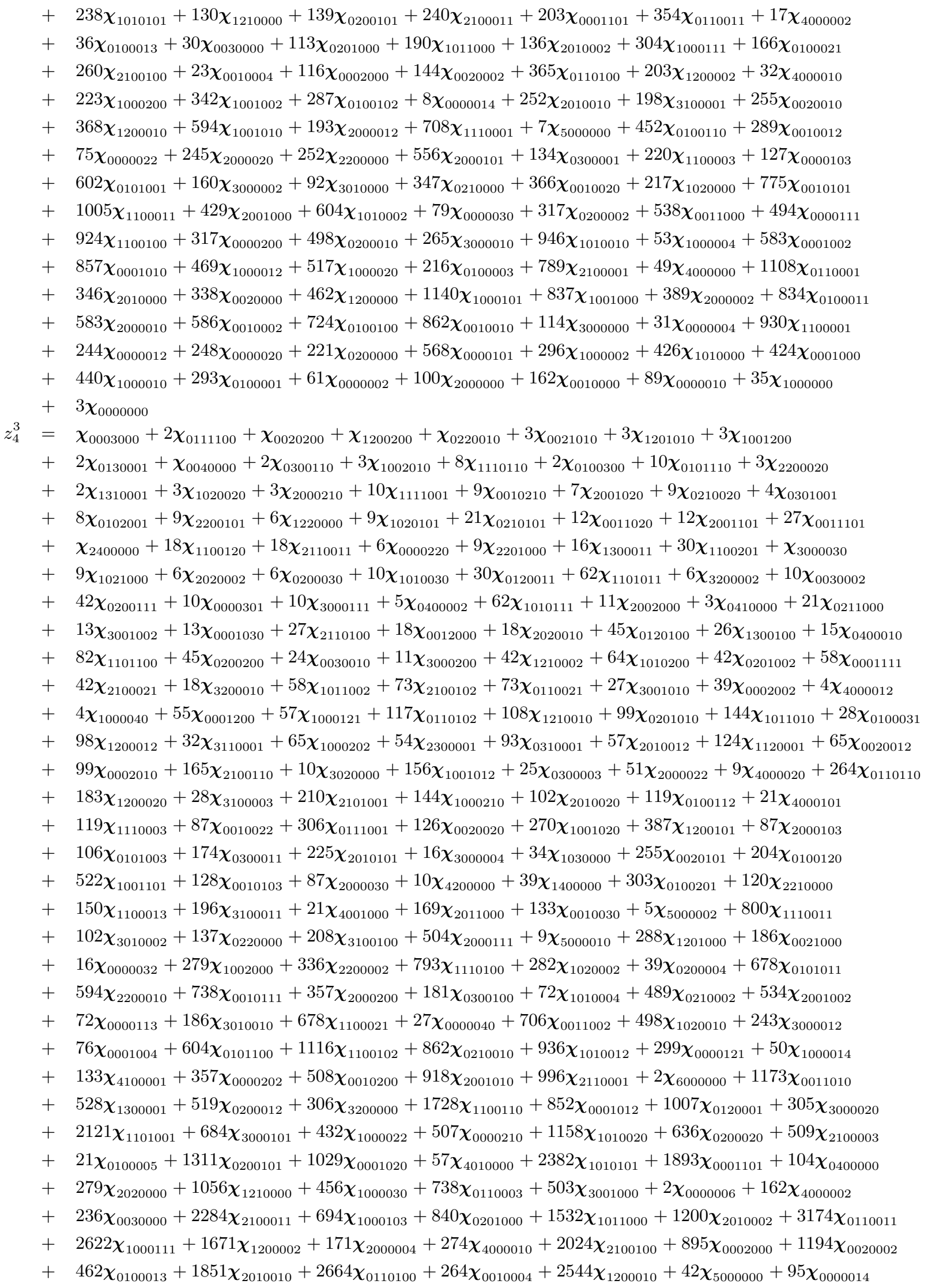




$$
+
$$$$
z_{1} z_{2} z_{5}
$$$$
=
$$

$$
+
$$

$$
+
$$

$$
+
$$

$$
+
$$

$$
+
$$$$
+
$$

$$
+
$$

$$
+
$$$$
z_{3}^{2} z_{5}
$$

$$
\begin{aligned}
& + \\
& + \\
& + \\
& + \\
& +
\end{aligned}
$$

$$
+
$$$$
+
$$

$1815 \chi_{0020010}+1594 \chi_{1000200}+2807 \chi_{1001002}+4050 \chi_{1001010}+1335 \chi_{0100021}+1467 \chi_{2000012}+2217 \chi_{0100102}$ $2160 \chi_{0010012}+2936 \chi_{0100110}+504 \chi_{0000022}+815 \chi_{0300001}+1599 \chi_{2000020}+1330 \chi_{3100001}+1536 \chi_{1100003}$ $4495 \chi_{1110001}+2262 \chi_{0010020}+470 \chi_{0000030}+1351 \chi_{2200000}+3652 \chi_{0101001}+526 \chi_{3010000}+3390 \chi_{2000101}$ $886 \chi_{3000002}+1827 \chi_{0210000}+1187 \chi_{1020000}+846 \chi_{0000103}+4622 \chi_{0010101}+318 \chi_{1000004}+2319 \chi_{2001000}$ $5700 \chi_{1100011}+1308 \chi_{3000010}+2793 \chi_{0011000}+4668 \chi_{1100100}+2682 \chi_{0000111}+1674 \chi_{0200002}+3220 \chi_{1010002}$ $1522 \chi_{0000200}+4590 \chi_{1010010}+3561 \chi_{2100001}+3004 \chi_{0001002}+2350 \chi_{0200010}+2303 \chi_{1000012}+4074 \chi_{0001010}$ $209 \chi_{4000000}+987 \chi_{0100003}+2292 \chi_{1000020}+1398 \chi_{2010000}+4927 \chi_{0110001}+4917 \chi_{1000101}+1853 \chi_{1200000}$ $131 \chi_{0000004}+3432 \chi_{0100011}+1517 \chi_{2000002}+1379 \chi_{0020000}+2292 \chi_{0010002}+3288 \chi_{1001000}+2187 \chi_{2000010}$ $879 \chi_{0000012}+361 \chi_{3000000}+2781 \chi_{0100100}+3168 \chi_{0010010}+3242 \chi_{1100001}+702 \chi_{0200000}+875 \chi_{0000020}$ $1372 \chi_{1010000}+1905 \chi_{0000101}+924 \chi_{1000002}+1366 \chi_{0001000}+1311 \chi_{1000010}+276 \chi_{2000000}+835 \chi_{0100001}$ $424 \chi_{0010000}+151 \chi_{0000002}+228 \chi_{0000010}+75 \chi_{1000000}+7 \chi_{0000000}$ $\chi_{2000100}+\chi_{0010100}+2 \chi_{1100010}+2 \chi_{0000110}+\chi_{0200001}+2 \chi_{1010001}+2 \chi_{2100000}+3 \chi_{0001001}$ $4 \chi_{0110000}+4 \chi_{1000011}+3 \chi_{0100002}+6 \chi_{1000100}+7 \chi_{0100010}+4 \chi_{2000001}+8 \chi_{0010001}+\chi_{0000003}$ $8 \chi_{1100000}+6 \chi_{0000011}+8 \chi_{0000100}+7 \chi_{1000001}+4 \chi_{0100000}+2 \chi_{0000001}$ $\chi_{1100100}+\chi_{0200010}+\chi_{1010010}+\chi_{0000200}+2 \chi_{0001010}+2 \chi_{1000020}+\chi_{2100001}+3 \chi_{0110001}$ $4 \chi_{1000101}+\chi_{2010000}+2 \chi_{2000002}+3 \chi_{1200000}+2 \chi_{0020000}+5 \chi_{1001000}+5 \chi_{0100011}+5 \chi_{2000010}$ $7 \chi_{0100100}+4 \chi_{0010002}+10 \chi_{0010010}+3 \chi_{0000012}+14 \chi_{1100001}+5 \chi_{0000020}+6 \chi_{0200000}+12 \chi_{0000101}$ $2 \chi_{3000000}+9 \chi_{1010000}+9 \chi_{1000002}+12 \chi_{0001000}+16 \chi_{1000010}+6 \chi_{2000000}+14 \chi_{0100001}+10 \chi_{0010000}$ $5 \chi_{0000002}+8 \chi_{0000010}+4 \chi_{1000000}+\chi_{0000000}$ $\chi_{0200100}+\chi_{0001100}+2 \chi_{0110010}+\chi_{0020001}+2 \chi_{1000110}+3 \chi_{0100020}+2 \chi_{1200001}+2 \chi_{0300000}$ $3 \chi_{1001001}+5 \chi_{0100101}+2 \chi_{2000011}+4 \chi_{1110000}+6 \chi_{0010011}+6 \chi_{0101000}+3 \chi_{0000021}+4 \chi_{2000100}$ $6 \chi_{1100002}+8 \chi_{0010100}+6 \chi_{0000102}+16 \chi_{1100010}+2 \chi_{3000001}+11 \chi_{0000110}+3 \chi_{1000003}+10 \chi_{0200001}$ $14 \chi_{1010001}+11 \chi_{2100000}+20 \chi_{0001001}+18 \chi_{0110000}+22 \chi_{1000011}+25 \chi_{1000100}+16 \chi_{0100002}+16 \chi_{2000001}$ $4 \chi_{0000003}+26 \chi_{0100010}+30 \chi_{0010001}+24 \chi_{1100000}+19 \chi_{0000011}+22 \chi_{0000100}+20 \chi_{1000001}+11 \chi_{0100000}$ $5 \chi_{0000001}$

$\chi_{1010100}+\chi_{0001100}+\chi_{2100010}+\chi_{2010001}+2 \chi_{0110010}+2 \chi_{1200001}+3 \chi_{1000110}+\chi_{0300000}$

$2 \chi_{0020001}+4 \chi_{1001001}+\chi_{3100000}+2 \chi_{0100020}+4 \chi_{2000011}+5 \chi_{0100101}+7 \chi_{0010011}+6 \chi_{1110000}$

$7 \chi_{1100002}+6 \chi_{0101000}+3 \chi_{0000021}+5 \chi_{0000102}+6 \chi_{2000100}+10 \chi_{0010100}+18 \chi_{1100010}+4 \chi_{3000001}$ $12 \chi_{0000110}+18 \chi_{1010001}+4 \chi_{1000003}+13 \chi_{2100000}+10 \chi_{0200001}+20 \chi_{0001001}+23 \chi_{1000011}+21 \chi_{0110000}$ $15 \chi_{0100002}+27 \chi_{1000100}+27 \chi_{0100010}+19 \chi_{2000001}+30 \chi_{0010001}+4 \chi_{0000003}+25 \chi_{1100000}+19 \chi_{0000011}$ $19 \chi_{0000100}+20 \chi_{1000001}+10 \chi_{0100000}+5 \chi_{0000001}$

$\chi_{0110100}+\chi_{0020010}+\chi_{1000200}+\chi_{1200010}+\chi_{0300001}+2 \chi_{1001010}+3 \chi_{0100110}+3 \chi_{1110001}$ $4 \chi_{0101001}+\chi_{2000020}+2 \chi_{1020000}+3 \chi_{0010020}+3 \chi_{2000101}+6 \chi_{0010101}+10 \chi_{1100011}+4 \chi_{0200002}$ $2 \chi_{2200000}+4 \chi_{2001000}+\chi_{0000030}+5 \chi_{0210000}+7 \chi_{0000111}+7 \chi_{0011000}+\chi_{3000002}+15 \chi_{1100100}$ $7 \chi_{1010002}+6 \chi_{0000200}+12 \chi_{0200010}+9 \chi_{0001002}+3 \chi_{3000010}+18 \chi_{1010010}+9 \chi_{1000012}+21 \chi_{0001010}$ $19 \chi_{2100001}+16 \chi_{1000020}+32 \chi_{0110001}+6 \chi_{0100003}+39 \chi_{1000101}+\chi_{0000004}+\chi_{4000000}+36 \chi_{0100011}$ $12 \chi_{2010000}+20 \chi_{1200000}+13 \chi_{0020000}+17 \chi_{2000002}+38 \chi_{1001000}+31 \chi_{0010002}+33 \chi_{2000010}+39 \chi_{0100100}$ $17 \chi_{0000012}+9 \chi_{3000000}+53 \chi_{0010010}+70 \chi_{1100001}+20 \chi_{0000020}+41 \chi_{1010000}+21 \chi_{0200000}+52 \chi_{0000101}$ $34 \chi_{1000002}+46 \chi_{0001000}+56 \chi_{1000010}+46 \chi_{0100001}+12 \chi_{0000002}+17 \chi_{2000000}+30 \chi_{0010000}+21 \chi_{0000010}$ $10 \chi_{1000000}+\chi_{0000000}$

$\chi_{0020100}+\chi_{1001100}+\chi_{0100200}+2 \chi_{1110010}+2 \chi_{0101010}+2 \chi_{2000110}+2 \chi_{1020001}+\chi_{2200001}$ $4 \chi_{0010110}+4 \chi_{1100020}+3 \chi_{0000120}+3 \chi_{2001001}+3 \chi_{0210001}+5 \chi_{0011001}+10 \chi_{1100101}+4 \chi_{2110000}$ $2 \chi_{3000011}+4 \chi_{0000201}+2 \chi_{1300000}+6 \chi_{0120000}+6 \chi_{0200011}+12 \chi_{1101000}+12 \chi_{1010011}+4 \chi_{3000100}$

$10 \chi_{0200100}+9 \chi_{2100002}+13 \chi_{0001011}+18 \chi_{1010100}+9 \chi_{1000021}+14 \chi_{0110002}+23 \chi_{2100010}+16 \chi_{0001100}$

$+37 \chi_{0110010}+2 \chi_{4000001}+17 \chi_{1000102}+32 \chi_{1200001}+38 \chi_{1000110}+22 \chi_{2010001}+6 \chi_{2000003}+14 \chi_{0100012}$

$+26 \chi_{0100020}+12 \chi_{0010003}+24 \chi_{0020001}+62 \chi_{1001001}+10 \chi_{0300000}+14 \chi_{3100000}+46 \chi_{2000011}+58 \chi_{0100101}$ 


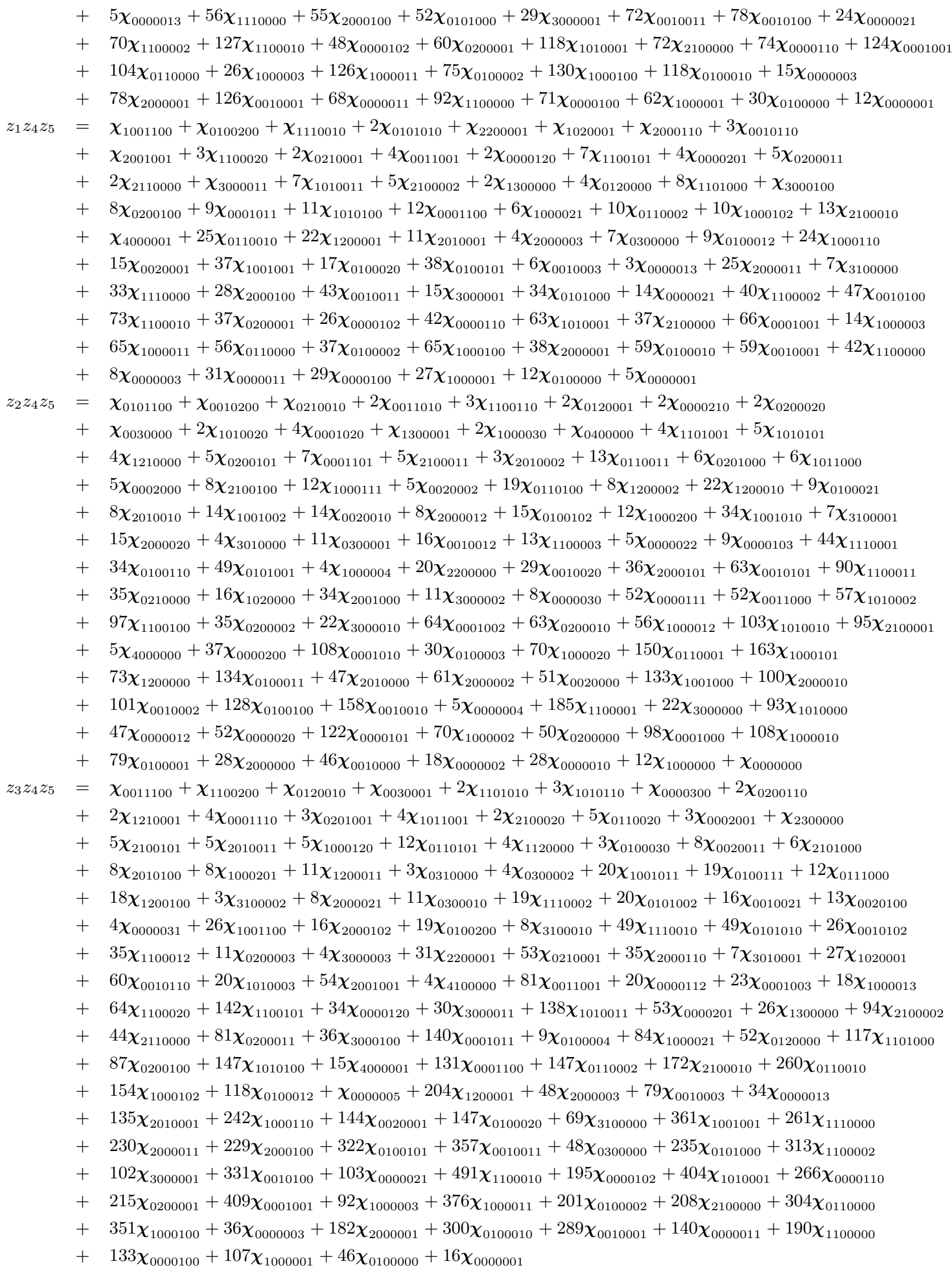




$$
\begin{aligned}
& z_{4}^{2} z_{5}=\chi_{0002100}+\chi_{0110200}+2 \chi_{0111010}+2 \chi_{0020110}+\chi_{1000300}+2 \chi_{1200110}+\chi_{0220001}+4 \chi_{1001110} \\
& +4 \chi_{0100210}+3 \chi_{0021001}+\chi_{0300020}+2 \chi_{0130000}+4 \chi_{1110020}+3 \chi_{1201001}+6 \chi_{0101020}+3 \chi_{1002001} \\
& +10 \chi_{1110101}+3 \chi_{2000120}+7 \chi_{0010120}+3 \chi_{0300101}+2 \chi_{1310000}+6 \chi_{1020011}+12 \chi_{0101101}+6 \chi_{2200011} \\
& +4 \chi_{0301000}+4 \chi_{2000201}+6 \chi_{1100030}+10 \chi_{1111000}+8 \chi_{0102000}+12 \chi_{0010201}+16 \chi_{0210011}+11 \chi_{2001011} \\
& +10 \chi_{1020100}+4 \chi_{0000130}+23 \chi_{0011011}+9 \chi_{2110002}+8 \chi_{1300002}+36 \chi_{1100111}+10 \chi_{2200100}+16 \chi_{0120002} \\
& +14 \chi_{0000211}+24 \chi_{0210100}+14 \chi_{2001100}+22 \chi_{1300010}+16 \chi_{0200021}+3 \chi_{3000021}+10 \chi_{0400001}+24 \chi_{1010021} \\
& +35 \chi_{1101002}+7 \chi_{3000102}+24 \chi_{2110010}+12 \chi_{2020001}+30 \chi_{0011100}+36 \chi_{1100200}+28 \chi_{0200102}+40 \chi_{1010102} \\
& +28 \chi_{0001021}+41 \chi_{0120010}+12 \chi_{0000300}+85 \chi_{1101010}+18 \chi_{0030001}+39 \chi_{0001102}+14 \chi_{3000110}+38 \chi_{2100012} \\
& +90 \chi_{1010110}+13 \chi_{1000031}+\chi_{4000003}+12 \chi_{3200001}+62 \chi_{0200110}+87 \chi_{0001110}+66 \chi_{0110012}+22 \chi_{3001001} \\
& +33 \chi_{1200003}+19 \chi_{2010003}+80 \chi_{1210001}+58 \chi_{1000112}+80 \chi_{0201001}+68 \chi_{2100020}+28 \chi_{2300000}+34 \chi_{0100022} \\
& +114 \chi_{1011001}+22 \chi_{0020003}+58 \chi_{1001003}+120 \chi_{0110020}+30 \chi_{2000013}+150 \chi_{2100101}+16 \chi_{3110000}+82 \chi_{0002001} \\
& +98 \chi_{1000120}+246 \chi_{0110101}+49 \chi_{0310000}+51 \chi_{0100103}+10 \chi_{4000011}+148 \chi_{1000201}+128 \chi_{2010011} \\
& +66 \chi_{1120000}+230 \chi_{1200011}+116 \chi_{2101000}+51 \chi_{0010013}+13 \chi_{4000100}+174 \chi_{0111000}+13 \chi_{0000023} \\
& +53 \chi_{0100030}+156 \chi_{0020011}+74 \chi_{3100002}+362 \chi_{1001011}+237 \chi_{1200100}+134 \chi_{2010100}+140 \chi_{2000021} \\
& +324 \chi_{1110002}+135 \chi_{3100010}+158 \chi_{0020100}+68 \chi_{0300002}+36 \chi_{1100004}+296 \chi_{0100111}+226 \chi_{0010021} \\
& +246 \chi_{2000102}+328 \chi_{1001100}+204 \chi_{0100200}+52 \chi_{0000031}+298 \chi_{0101002}+374 \chi_{0010102}+4 \chi_{5000001} \\
& +23 \chi_{0000104}+124 \chi_{0300010}+58 \chi_{3000003}+466 \chi_{1100012}+570 \chi_{1110010}+96 \chi_{3010001}+386 \chi_{2000110} \\
& +138 \chi_{0200003}+501 \chi_{0101010}+324 \chi_{2200001}+574 \chi_{0010110}+274 \chi_{1020001}+489 \chi_{0210001}+576 \chi_{1100020} \\
& +44 \chi_{4100000}+540 \chi_{2001001}+720 \chi_{0011001}+277 \chi_{3000011}+1202 \chi_{1100101}+8 \chi_{1000005}+246 \chi_{1010003} \\
& +236 \chi_{0000112}+187 \chi_{1300000}+250 \chi_{0001003}+271 \chi_{0000120}+354 \chi_{2110000}+194 \chi_{1000013}+405 \chi_{0000201} \\
& +366 \chi_{0120000}+1096 \chi_{1010011}+689 \chi_{2100002}+804 \chi_{1101000}+610 \chi_{0200011}+1031 \chi_{0001011}+538 \chi_{0200100} \\
& +571 \chi_{1000021}+270 \chi_{3000100}+974 \chi_{1010100}+1007 \chi_{0110002}+1067 \chi_{2100010}+100 \chi_{4000001}+86 \chi_{0100004} \\
& +810 \chi_{0001100}+1003 \chi_{1000102}+12 \chi_{0000005}+1516 \chi_{0110010}+762 \chi_{2010001}+778 \chi_{0020001}+1347 \chi_{1000110} \\
& +712 \chi_{0100012}+1082 \chi_{1200001}+1872 \chi_{1001001}+752 \chi_{0100020}+212 \chi_{0300000}+1574 \chi_{0100101}+283 \chi_{2000003} \\
& +334 \chi_{3100000}+1116 \chi_{2000011}+446 \chi_{0010003}+1182 \chi_{1110000}+176 \chi_{0000013}+991 \chi_{2000100}+1012 \chi_{0101000} \\
& +1660 \chi_{0010011}+442 \chi_{0000021}+1383 \chi_{0010100}+1356 \chi_{1100002}+1948 \chi_{1100010}+794 \chi_{0000102}+1002 \chi_{0000110} \\
& +394 \chi_{3000001}+779 \chi_{0200001}+1494 \chi_{1010001}+1461 \chi_{0001001}+690 \chi_{2100000}+348 \chi_{1000003}+1263 \chi_{1000011} \\
& +990 \chi_{0110000}+631 \chi_{0100002}+1096 \chi_{1000100}+890 \chi_{0100010}+522 \chi_{2000001}+808 \chi_{0010001}+492 \chi_{1100000} \\
& +101 \chi_{0000003}+368 \chi_{0000011}+332 \chi_{0000100}+246 \chi_{1000001}+98 \chi_{0100000}+32 \chi_{0000001} \\
& z_{1} z_{5}^{2}=\chi_{1000200}+\chi_{1001010}+2 \chi_{0100110}+\chi_{2000020}+\chi_{1110001}+2 \chi_{0101001}+2 \chi_{0010020}+\chi_{2000101} \\
& +\chi_{1020000}+4 \chi_{0010101}+6 \chi_{1100011}+\chi_{3000002}+\chi_{2200000}+\chi_{2001000}+\chi_{0000030}+6 \chi_{0000111} \\
& +2 \chi_{0210000}+4 \chi_{0011000}+3 \chi_{0200002}+8 \chi_{1100100}+4 \chi_{1010002}+6 \chi_{0001002}+7 \chi_{1000012}+5 \chi_{0000200} \\
& +7 \chi_{0200010}+\chi_{3000010}+10 \chi_{1010010}+4 \chi_{0100003}+\chi_{0000004}+14 \chi_{0001010}+12 \chi_{1000020}+10 \chi_{2100001} \\
& +20 \chi_{0110001}+25 \chi_{1000101}+\chi_{4000000}+12 \chi_{1200000}+26 \chi_{0100011}+6 \chi_{2010000}+8 \chi_{0020000}+12 \chi_{2000002} \\
& +21 \chi_{0010002}+22 \chi_{1001000}+20 \chi_{2000010}+27 \chi_{0100100}+13 \chi_{0000012}+36 \chi_{0010010}+6 \chi_{3000000}+46 \chi_{1100001} \\
& +16 \chi_{0000020}+26 \chi_{1010000}+15 \chi_{0200000}+35 \chi_{0000101}+25 \chi_{1000002}+29 \chi_{0001000}+38 \chi_{1000010}+31 \chi_{0100001} \\
& +10 \chi_{0000002}+12 \chi_{2000000}+19 \chi_{0010000}+14 \chi_{0000010}+8 \chi_{1000000}+\chi_{0000000} \\
& z_{2} z_{5}^{2}=\chi_{0100200}+\chi_{0101010}+2 \chi_{0010110}+2 \chi_{1100020}+\chi_{0210001}+2 \chi_{0011001}+4 \chi_{1100101}+3 \chi_{0000120} \\
& +2 \chi_{0120000}+4 \chi_{1010011}+3 \chi_{0000201}+4 \chi_{0200011}+\chi_{1300000}+8 \chi_{0001011}+3 \chi_{2100002}+6 \chi_{1000021} \\
& +4 \chi_{1101000}+7 \chi_{0110002}+10 \chi_{1000102}+3 \chi_{2000003}+6 \chi_{1010100}+6 \chi_{0200100}+8 \chi_{0001100}+7 \chi_{2100010} \\
& +19 \chi_{0110010}+10 \chi_{0100012}+16 \chi_{1200001}+6 \chi_{0300000}+7 \chi_{0010003}+6 \chi_{2010001}+10 \chi_{0020001} \\
& +20 \chi_{1000110}+18 \chi_{0100020}+30 \chi_{1001001}+4 \chi_{3100000}+4 \chi_{0000013}+36 \chi_{0100101}+22 \chi_{2000011}+44 \chi_{0010011} \\
& +24 \chi_{1110000}+30 \chi_{0101000}+25 \chi_{2000100}+42 \chi_{1100002}+18 \chi_{0000021}+34 \chi_{0000102}+42 \chi_{0010100}+18 \chi_{1000003} \\
& +73 \chi_{1100010}+48 \chi_{0000110}+12 \chi_{3000001}+64 \chi_{1010001}+38 \chi_{2100000}+38 \chi_{0200001}+77 \chi_{0001001}+81 \chi_{1000011} \\
& +52 \chi_{0100002}+60 \chi_{0110000}+80 \chi_{1000100}+46 \chi_{2000001}+77 \chi_{0100010}+82 \chi_{0010001}+11 \chi_{0000003}+48 \chi_{0000011} \\
& +58 \chi_{1100000}+47 \chi_{0000100}+42 \chi_{1000001}+22 \chi_{0100000}+8 \chi_{0000001}
\end{aligned}
$$




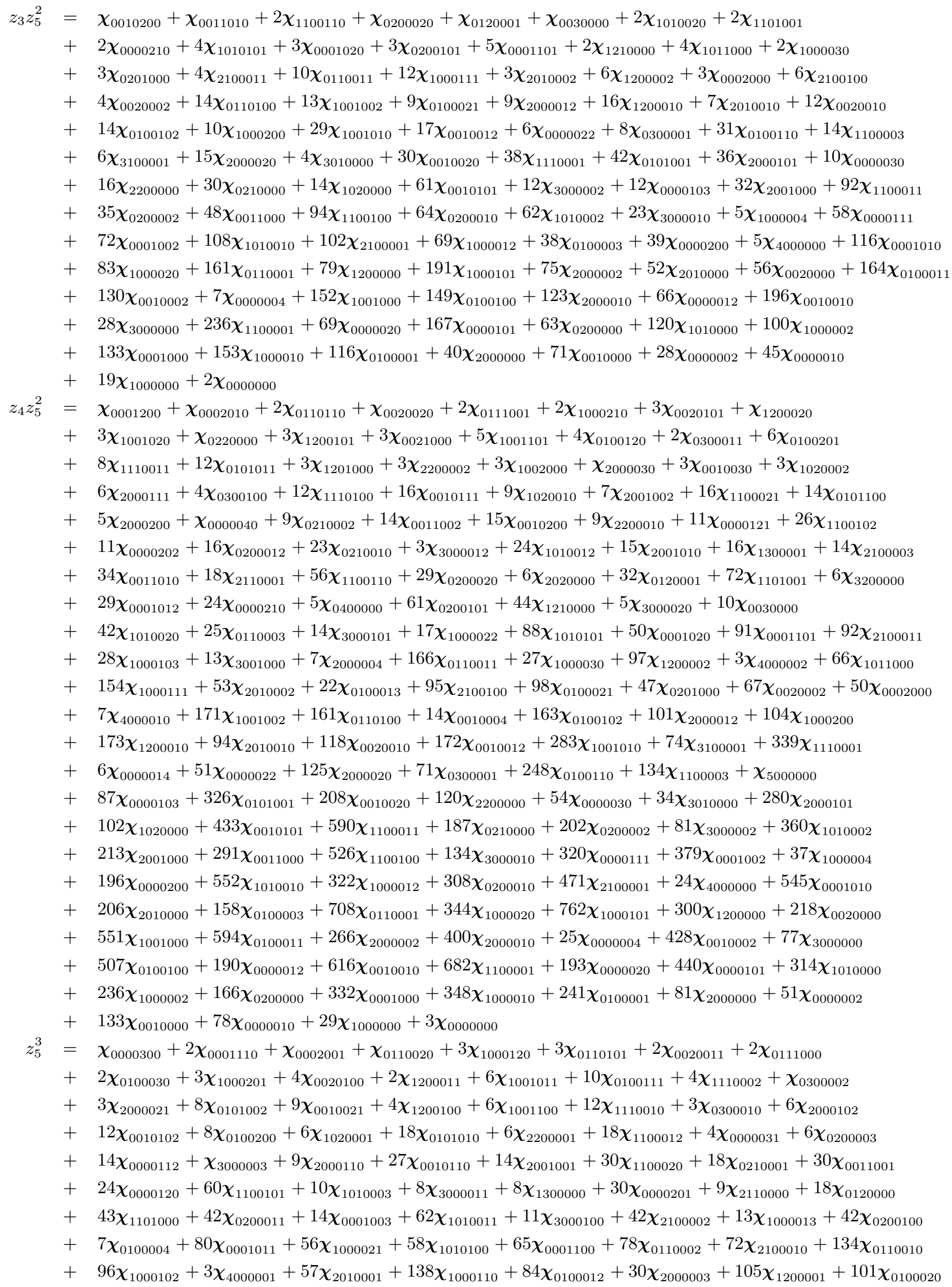




$$
\begin{aligned}
& +\chi_{0000005}+72 \chi_{0020001}+198 \chi_{1001001}+28 \chi_{0300000}+204 \chi_{0100101}+28 \chi_{3100000}+134 \chi_{1110000}+58 \chi_{0010003} \\
& +138 \chi_{2000011}+30 \chi_{0000013}+239 \chi_{0010011}+84 \chi_{0000021}+139 \chi_{0101000}+216 \chi_{1100002}+132 \chi_{2000100} \\
& +58 \chi_{3000001}+204 \chi_{0010100}+324 \chi_{1100010}+150 \chi_{0200001}+156 \chi_{0000102}+270 \chi_{1010001}+198 \chi_{0000110} \\
& +140 \chi_{2100000}+304 \chi_{0001001}+77 \chi_{1000003}+216 \chi_{0110000}+300 \chi_{1000011}+174 \chi_{0100002}+270 \chi_{1000100} \\
& +34 \chi_{0000003}+246 \chi_{0100010}+144 \chi_{2000001}+246 \chi_{0010001}+162 \chi_{1100000}+132 \chi_{0000011}+128 \chi_{0000100} \\
& +102 \chi_{1000001}+47 \chi_{0100000}+17 \chi_{0000001} \\
& z_{1}^{2} z_{6}=\chi_{2000010}+\chi_{0010010}+2 \chi_{1100001}+\chi_{0200000}+\chi_{0000020}+2 \chi_{0000101}+3 \chi_{1000002}+2 \chi_{1010000} \\
& +3 \chi_{0001000}+5 \chi_{1000010}+6 \chi_{0100001}+3 \chi_{2000000}+3 \chi_{0000002}+5 \chi_{0010000}+6 \chi_{0000010}+3 \chi_{1000000}+\chi_{0000000} \\
& z_{1} z_{2} z_{6}=\chi_{1100010}+\chi_{0200001}+\chi_{1010001}+\chi_{0000110}+2 \chi_{0001001}+3 \chi_{1000011}+\chi_{2100000}+3 \chi_{0100002} \\
& +3 \chi_{0110000}+4 \chi_{1000100}+4 \chi_{2000001}+\chi_{0000003}+6 \chi_{0100010}+8 \chi_{0010001}+8 \chi_{1100000}+7 \chi_{0000011} \\
& +8 \chi_{0000100}+10 \chi_{1000001}+6 \chi_{0100000}+4 \chi_{0000001} \\
& z_{2}^{2} z_{6}=\chi_{0200010}+\chi_{0001010}+2 \chi_{0110001}+\chi_{0020000}+\chi_{1000020}+2 \chi_{1000101}+4 \chi_{0100011}+\chi_{2000002} \\
& +2 \chi_{1200000}+3 \chi_{0010002}+3 \chi_{1001000}+5 \chi_{0100100}+3 \chi_{2000010}+7 \chi_{0010010}+12 \chi_{1100001}+\chi_{3000000} \\
& +3 \chi_{0000012}+4 \chi_{0000020}+11 \chi_{0000101}+5 \chi_{0200000}+9 \chi_{1000002}+8 \chi_{1010000}+11 \chi_{0001000}+16 \chi_{1000010} \\
& +6 \chi_{2000000}+16 \chi_{0100001}+12 \chi_{0010000}+6 \chi_{0000002}+11 \chi_{0000010}+6 \chi_{1000000}+\chi_{0000000} \\
& z_{1} z_{3} z_{6}=\chi_{1010010}+\chi_{0001010}+\chi_{1000020}+\chi_{2100001}+2 \chi_{0110001}+3 \chi_{1000101}+\chi_{2010000}+2 \chi_{2000002} \\
& +2 \chi_{1200000}+3 \chi_{0100011}+2 \chi_{0020000}+4 \chi_{1001000}+5 \chi_{2000010}+5 \chi_{0100100}+4 \chi_{0010002}+8 \chi_{0010010} \\
& +13 \chi_{1100001}+2 \chi_{0000012}+4 \chi_{0000020}+10 \chi_{0000101}+2 \chi_{3000000}+5 \chi_{0200000}+10 \chi_{1010000}+9 \chi_{1000002} \\
& +12 \chi_{0001000}+16 \chi_{1000010}+7 \chi_{2000000}+15 \chi_{0100001}+11 \chi_{0010000}+5 \chi_{0000002}+9 \chi_{0000010}+5 \chi_{1000000} \\
& +\chi_{0000000} \\
& z_{2} z_{3} z_{6}=\chi_{0110010}+\chi_{1000110}+\chi_{0020001}+\chi_{0100020}+\chi_{1200001}+2 \chi_{1001001}+\chi_{0300000}+3 \chi_{1110000} \\
& +3 \chi_{0100101}+4 \chi_{0101000}+2 \chi_{2000011}+4 \chi_{0010011}+5 \chi_{1100002}+2 \chi_{0000021}+3 \chi_{2000100}+6 \chi_{0010100} \\
& +12 \chi_{1100010}+4 \chi_{0000102}+2 \chi_{3000001}+8 \chi_{0000110}+3 \chi_{1000003}+8 \chi_{0200001}+13 \chi_{1010001}+10 \chi_{2100000} \\
& +16 \chi_{0001001}+17 \chi_{0110000}+19 \chi_{1000011}+23 \chi_{1000100}+14 \chi_{0100002}+17 \chi_{2000001}+24 \chi_{0100010}+4 \chi_{0000003} \\
& +29 \chi_{0010001}+25 \chi_{1100000}+19 \chi_{0000011}+21 \chi_{0000100}+22 \chi_{1000001}+12 \chi_{0100000}+6 \chi_{0000001} \\
& z_{3}^{2} z_{6}=\chi_{0020010}+\chi_{1001010}+\chi_{0100110}+\chi_{2000020}+2 \chi_{1110001}+2 \chi_{0101001}+\chi_{0010020}+2 \chi_{2000101} \\
& +\chi_{2200000}+4 \chi_{0010101}+2 \chi_{1020000}+6 \chi_{1100011}+3 \chi_{2001000}+3 \chi_{0210000}+3 \chi_{0200002}+\chi_{3000002} \\
& +5 \chi_{0011000}+\chi_{0000030}+4 \chi_{0000111}+10 \chi_{1100100}+6 \chi_{1010002}+6 \chi_{0001002}+3 \chi_{3000010}+7 \chi_{1000012} \\
& +7 \chi_{0200010}+4 \chi_{0000200}+14 \chi_{1010010}+15 \chi_{0001010}+16 \chi_{2100001}+4 \chi_{0100003}+\chi_{4000000}+12 \chi_{1000020} \\
& +25 \chi_{0110001}+11 \chi_{2010000}+31 \chi_{1000101}+16 \chi_{1200000}+28 \chi_{0100011}+16 \chi_{2000002}+13 \chi_{0020000}+33 \chi_{1001000} \\
& +31 \chi_{2000010}+26 \chi_{0010002}+\chi_{0000004}+33 \chi_{0100100}+10 \chi_{3000000}+14 \chi_{0000012}+46 \chi_{0010010}+19 \chi_{0000020} \\
& +64 \chi_{1100001}+46 \chi_{0000101}+20 \chi_{0200000}+33 \chi_{1000002}+40 \chi_{1010000}+44 \chi_{0001000}+55 \chi_{1000010}+45 \chi_{0100001} \\
& +13 \chi_{0000002}+19 \chi_{2000000}+30 \chi_{0010000}+23 \chi_{0000010}+11 \chi_{1000000}+2 \chi_{0000000} \\
& z_{1} z_{4} z_{6}=\chi_{1001010}+\chi_{0100110}+\chi_{1110001}+2 \chi_{0101001}+\chi_{2200000}+\chi_{0010020}+\chi_{2000101}+\chi_{1020000} \\
& +3 \chi_{0010101}+\chi_{2001000}+4 \chi_{1100011}+2 \chi_{0210000}+2 \chi_{0200002}+4 \chi_{0011000}+3 \chi_{0000111}+3 \chi_{1010002} \\
& +7 \chi_{1100100}+\chi_{3000010}+5 \chi_{0001002}+3 \chi_{1000012}+6 \chi_{0200010}+4 \chi_{0000200}+8 \chi_{1010010}+10 \chi_{0001010} \\
& +8 \chi_{2100001}+2 \chi_{0100003}+7 \chi_{1000020}+17 \chi_{0110001}+18 \chi_{1000101}+7 \chi_{2000002}+10 \chi_{1200000}+5 \chi_{2010000} \\
& +17 \chi_{0100011}+13 \chi_{0010002}+7 \chi_{0020000}+20 \chi_{1001000}+13 \chi_{2000010}+21 \chi_{0100100}+27 \chi_{0010010}+6 \chi_{0000012} \\
& +4 \chi_{3000000}+8 \chi_{0000020}+33 \chi_{1100001}+23 \chi_{0000101}+12 \chi_{0200000}+12 \chi_{1000002}+19 \chi_{1010000}+21 \chi_{0001000} \\
& +24 \chi_{1000010}+18 \chi_{0100001}+6 \chi_{2000000}+4 \chi_{0000002}+12 \chi_{0010000}+6 \chi_{0000010}+3 \chi_{1000000} \\
& z_{2} z_{4} z_{6}=\chi_{0101010}+\chi_{0010110}+\chi_{0210001}+\chi_{1100020}+2 \chi_{0011001}+3 \chi_{1100101}+2 \chi_{0120000}+\chi_{0000120} \\
& +3 \chi_{0200011}+3 \chi_{1010011}+2 \chi_{0000201}+\chi_{1300000}+5 \chi_{0001011}+3 \chi_{1000021}+2 \chi_{2100002}+4 \chi_{1101000} \\
& +6 \chi_{0110002}+6 \chi_{1000102}+5 \chi_{1010100}+5 \chi_{0200100}+7 \chi_{0001100}+2 \chi_{2000003}+6 \chi_{2100010}+15 \chi_{0110010} \\
& +6 \chi_{0100012}+14 \chi_{1200001}+5 \chi_{2010001}+4 \chi_{0010003}+5 \chi_{0300000}+9 \chi_{0020001}+14 \chi_{1000110}+11 \chi_{0100020} \\
& +24 \chi_{1001001}+2 \chi_{0000013}+27 \chi_{0100101}+3 \chi_{3100000}+15 \chi_{2000011}+30 \chi_{0010011}+29 \chi_{1100002} \\
& +22 \chi_{1110000}+10 \chi_{0000021}+19 \chi_{2000100}+25 \chi_{0101000}+20 \chi_{0000102}+35 \chi_{0010100}+55 \chi_{1100010}+33 \chi_{0000110}
\end{aligned}
$$




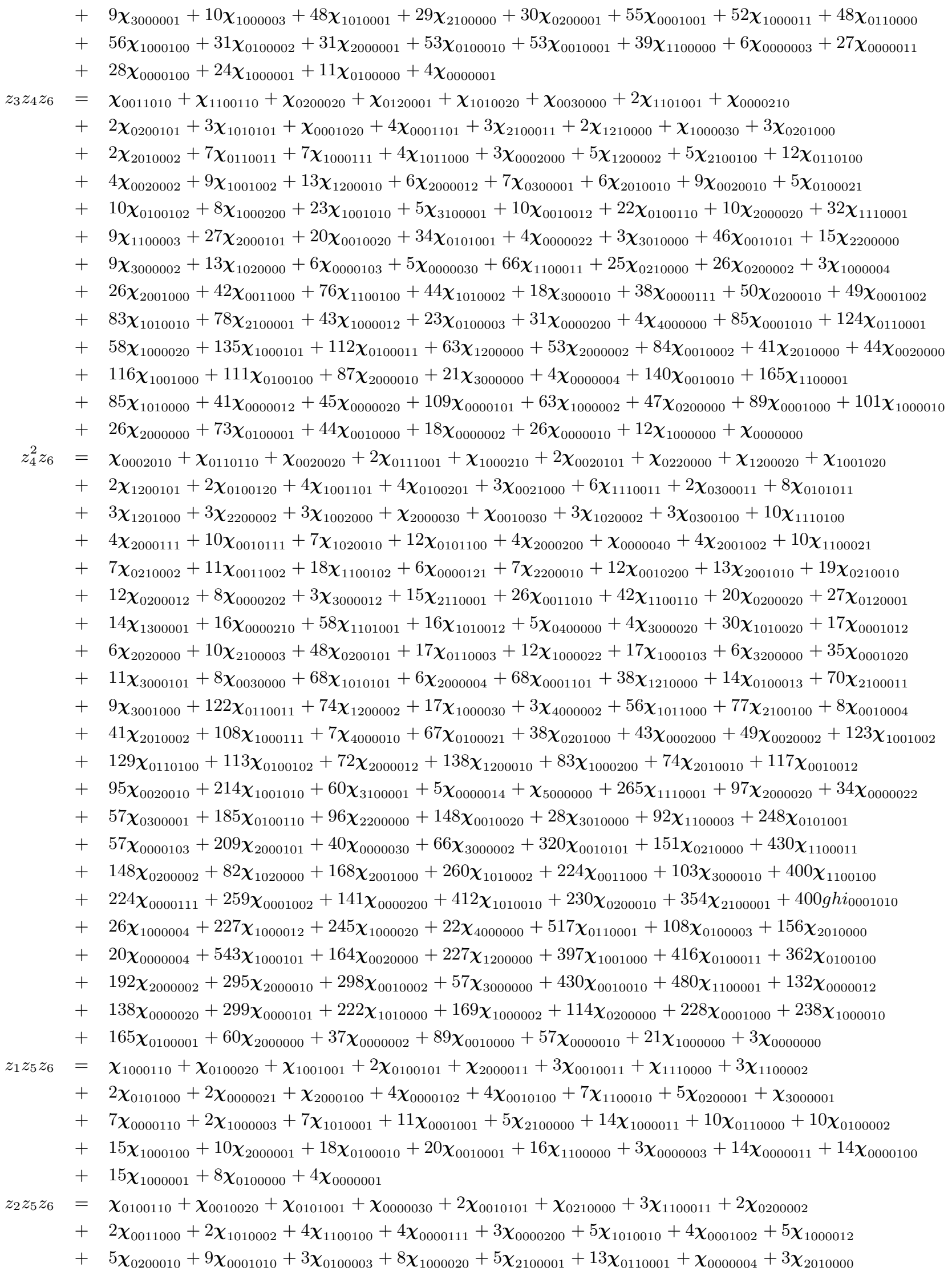


$8 \chi_{1200000}+18 \chi_{1000101}+8 \chi_{2000002}+5 \chi_{0020000}+21 \chi_{0100011}+16 \chi_{1001000}+15 \chi_{2000010}+21 \chi_{0100100}$

$+17 \chi_{0010002}+29 \chi_{0010010}+39 \chi_{1100001}+12 \chi_{0000012}+14 \chi_{0000020}+33 \chi_{0000101}+4 \chi_{3000000}+13 \chi_{0200000}$

$+22 \chi_{1010000}+23 \chi_{1000002}+28 \chi_{0001000}+37 \chi_{1000010}+11 \chi_{2000000}+32 \chi_{0100001}+10 \chi_{0000002}+21 \chi_{0010000}$

$+16 \chi_{0000010}+8 \chi_{1000000}+\chi_{0000000}$

$z_{3} z_{5} z_{6}=\chi_{0010110}+\chi_{1100020}+\chi_{0011001}+\chi_{0000120}+2 \chi_{1100101}+3 \chi_{1010011}+\chi_{0120000}+2 \chi_{0000201}$

$+2 \chi_{0200011}+2 \chi_{1101000}+4 \chi_{0001011}+2 \chi_{2100002}+4 \chi_{1000021}+5 \chi_{0110002}+3 \chi_{0200100}+4 \chi_{1010100}$

$+5 \chi_{0001100}+7 \chi_{1000102}+5 \chi_{2100010}+12 \chi_{0110010}+3 \chi_{2000003}+14 \chi_{1000110}+7 \chi_{0100012}+5 \chi_{2010001}$

$8 \chi_{0020001}+5 \chi_{0010003}+11 \chi_{1200001}+22 \chi_{1001001}+18 \chi_{2000011}+4 \chi_{0000013}+12 \chi_{0100020}+4 \chi_{0300000}$

$26 \chi_{0100101}+34 \chi_{0010011}+3 \chi_{3100000}+19 \chi_{1110000}+22 \chi_{0101000}+34 \chi_{1100002}+14 \chi_{0000021}+20 \chi_{2000100}$

$27 \chi_{0000102}+16 \chi_{1000003}+35 \chi_{0010100}+60 \chi_{1100010}+33 \chi_{0200001}+11 \chi_{3000001}+56 \chi_{1010001}+34 \chi_{2100000}$

$40 \chi_{0000110}+65 \chi_{0001001}+55 \chi_{0110000}+73 \chi_{1000011}+72 \chi_{1000100}+46 \chi_{0100002}+45 \chi_{2000001}+12 \chi_{0000003}$ $71 \chi_{0100010}+77 \chi_{0010001}+46 \chi_{0000011}+57 \chi_{1100000}+45 \chi_{0000100}+43 \chi_{1000001}+21 \chi_{0100000}+9 \chi_{0000001}$ $\chi_{0001110}+\chi_{0002001}+\chi_{0110020}+\chi_{1000120}+2 \chi_{0110101}+2 \chi_{0020011}+2 \chi_{0111000}+\chi_{0100030}$

$+3 \chi_{0020100}+2 \chi_{1000201}+2 \chi_{1200011}+\chi_{0300002}+4 \chi_{1001011}+4 \chi_{1110002}+3 \chi_{1200100}+6 \chi_{0100111}$

$+5 \chi_{1001100}+6 \chi_{0101002}+2 \chi_{2000021}+5 \chi_{0010021}+6 \chi_{0100200}+10 \chi_{1110010}+3 \chi_{0300010}+14 \chi_{0101010}$

$+6 \chi_{1020001}+3 \chi_{2000102}+6 \chi_{2200001}+2 \chi_{0000031}+9 \chi_{0010102}+7 \chi_{2000110}+19 \chi_{0010110}+12 \chi_{1100012}$

$8 \chi_{0000112}+21 \chi_{1100020}+11 \chi_{2001001}+14 \chi_{0000120}+16 \chi_{0210001}+\chi_{3000003}+5 \chi_{0200003}+25 \chi_{0011001}$

$9 \chi_{2110000}+46 \chi_{1100101}+7 \chi_{1010003}+21 \chi_{0000201}+8 \chi_{1300000}+16 \chi_{0120000}+32 \chi_{0200011}+8 \chi_{0001003}$ $8 \chi_{1000013}+37 \chi_{1101000}+6 \chi_{3000011}+46 \chi_{1010011}+32 \chi_{2100002}+56 \chi_{0001011}+33 \chi_{0200100}+7 \chi_{3000100}$

$4 \chi_{0100004}+48 \chi_{1010100}+57 \chi_{2100010}+36 \chi_{1000021}+52 \chi_{0001100}+59 \chi_{0110002}+105 \chi_{0110010}+64 \chi_{1000102}$ 


$$
\begin{aligned}
& z_{3} z_{6}^{2}=\chi_{0010020}+\chi_{0010101}+2 \chi_{1100011}+\chi_{0200002}+\chi_{0011000}+2 \chi_{0000111}+2 \chi_{1010002}+2 \chi_{1100100} \\
& +3 \chi_{0001002}+4 \chi_{1010010}+4 \chi_{1000012}+3 \chi_{0100003}+2 \chi_{0000200}+3 \chi_{0200010}+5 \chi_{0001010}+\chi_{0000004} \\
& +4 \chi_{2100001}+3 \chi_{2010000}+6 \chi_{1000020}+10 \chi_{0110001}+6 \chi_{1200000}+14 \chi_{1000101}+8 \chi_{2000002}+4 \chi_{0020000} \\
& +16 \chi_{0100011}+13 \chi_{1001000}+13 \chi_{2000010}+15 \chi_{0010002}+16 \chi_{0100100}+26 \chi_{0010010}+36 \chi_{1100001}+11 \chi_{0000012} \\
& +11 \chi_{0000020}+13 \chi_{0200000}+30 \chi_{0000101}+4 \chi_{3000000}+22 \chi_{1010000}+23 \chi_{1000002}+26 \chi_{0001000}+37 \chi_{1000010} \\
& +12 \chi_{2000000}+32 \chi_{0100001}+23 \chi_{0010000}+11 \chi_{0000002}+16 \chi_{0000010}+9 \chi_{1000000}+\chi_{0000000} \\
& z_{4} z_{6}^{2}=\chi_{0001020}+\chi_{0001101}+2 \chi_{0110011}+\chi_{0002000}+\chi_{0020002}+2 \chi_{1000111}+2 \chi_{0100021}+2 \chi_{0110100} \\
& +\chi_{1200002}+3 \chi_{0020010}+2 \chi_{1000200}+3 \chi_{1001002}+3 \chi_{1200010}+2 \chi_{0300001}+4 \chi_{0100102}+\chi_{2000012} \\
& +5 \chi_{1001010}+8 \chi_{1110001}+8 \chi_{0100110}+12 \chi_{0101001}+5 \chi_{0010012}+4 \chi_{1100003}+3 \chi_{2000020}+\chi_{0000022} \\
& +7 \chi_{0010020}+3 \chi_{0000103}+3 \chi_{1020000}+6 \chi_{2000101}+18 \chi_{0010101}+3 \chi_{2200000}+3 \chi_{0000030}+7 \chi_{2001000} \\
& +26 \chi_{1100011}+9 \chi_{0210000}+18 \chi_{0000111}+13 \chi_{0200002}+\chi_{1000004}+14 \chi_{0011000}+2 \chi_{3000002}+28 \chi_{1100100} \\
& +18 \chi_{1010002}+3 \chi_{3000010}+13 \chi_{0000200}+23 \chi_{0001002}+21 \chi_{0200010}+32 \chi_{1010010}+42 \chi_{0001010}+21 \chi_{1000012} \\
& +30 \chi_{2100001}+12 \chi_{0100003}+26 \chi_{1000020}+59 \chi_{0110001}+66 \chi_{1000101}+24 \chi_{2000002}+\chi_{4000000}+31 \chi_{1200000} \\
& +15 \chi_{2010000}+23 \chi_{0020000}+55 \chi_{1001000}+60 \chi_{0100011}+47 \chi_{0010002}+2 \chi_{0000004}+61 \chi_{0100100}+21 \chi_{0000012} \\
& +44 \chi_{2000010}+75 \chi_{0010010}+28 \chi_{0000020}+9 \chi_{3000000}+92 \chi_{1100001}+48 \chi_{1010000}+27 \chi_{0200000}+63 \chi_{0000101} \\
& +57 \chi_{0001000}+37 \chi_{1000002}+58 \chi_{1000010}+17 \chi_{2000000}+45 \chi_{0100001}+26 \chi_{0010000}+9 \chi_{0000002}+17 \chi_{0000010} \\
& +6 \chi_{1000000}+\chi_{0000000} \\
& z_{5} z_{6}^{2}=\chi_{0000120}+\chi_{0000201}+2 \chi_{0001011}+\chi_{0110002}+2 \chi_{0001100}+2 \chi_{1000021}+3 \chi_{0110010}+3 \chi_{1000102} \\
& +4 \chi_{0100012}+2 \chi_{0020001}+5 \chi_{1000110}+\chi_{2000003}+2 \chi_{1200001}+6 \chi_{0100020}+6 \chi_{1001001}+12 \chi_{0100101} \\
& +3 \chi_{0010003}+4 \chi_{1110000}+6 \chi_{2000011}+2 \chi_{0000013}+16 \chi_{0010011}+\chi_{0300000}+10 \chi_{0000021}+8 \chi_{0101000} \\
& +6 \chi_{2000100}+16 \chi_{1100002}+14 \chi_{0010100}+16 \chi_{0000102}+26 \chi_{1100010}+3 \chi_{3000001}+16 \chi_{0200001}+24 \chi_{0000110} \\
& +10 \chi_{1000003}+24 \chi_{1010001}+36 \chi_{0001001}+14 \chi_{2100000}+28 \chi_{0110000}+43 \chi_{1000011}+30 \chi_{0100002}+40 \chi_{1000100} \\
& +45 \chi_{0100010}+26 \chi_{2000001}+48 \chi_{0010001}+8 \chi_{0000003}+36 \chi_{1100000}+34 \chi_{0000011}+33 \chi_{0000100}+31 \chi_{1000001} \\
& +16 \chi_{0100000}+8 \chi_{0000001} \\
& z_{6}^{3}=\chi_{0000030}+2 \chi_{0000111}+\chi_{0001002}+\chi_{0000200}+3 \chi_{1000012}+3 \chi_{0001010}+2 \chi_{0110001}+2 \chi_{0100003}+\chi_{0020000} \\
& +3 \chi_{1000020}+6 \chi_{1000101}+10 \chi_{0100011}+3 \chi_{2000002}+\chi_{0000004}+9 \chi_{0010002}+\chi_{1200000}+3 \chi_{1001000} \\
& +8 \chi_{0100100}+6 \chi_{2000010}+12 \chi_{0010010}+9 \chi_{0000012}+18 \chi_{1100001}+11 \chi_{0000020}+6 \chi_{0200000}+21 \chi_{0000101} \\
& +18 \chi_{1000002}+\chi_{3000000}+10 \chi_{1010000}+17 \chi_{0001000}+25 \chi_{1000010}+25 \chi_{0100001}+10 \chi_{0000002}+9 \chi_{2000000} \\
& +16 \chi_{0010000}+18 \chi_{0000010}+8 \chi_{1000000}+2 \chi_{0000000} \\
& z_{1}^{2} z_{7}=\chi_{2000001}+\chi_{0010001}+2 \chi_{1100000}+\chi_{0000011}+2 \chi_{0000100}+4 \chi_{1000001}+3 \chi_{0100000}+3 \chi_{0000001} \\
& z_{1} z_{2} z_{7}=\chi_{1100001}+\chi_{0200000}+\chi_{1010000}+\chi_{0000101}+2 \chi_{0001000}+\chi_{1000002}+3 \chi_{1000010}+4 \chi_{0100001} \\
& +2 \chi_{0000002}+2 \chi_{2000000}+4 \chi_{0010000}+4 \chi_{0000010}+3 \chi_{1000000}+\chi_{0000000} \\
& z_{2}^{2} z_{7}=\chi_{0200001}+\chi_{0001001}+2 \chi_{0110000}+\chi_{1000011}+\chi_{0100002}+2 \chi_{1000100}+\chi_{0000003}+4 \chi_{0100010} \\
& +2 \chi_{2000001}+4 \chi_{0010001}+6 \chi_{1100000}+4 \chi_{0000011}+5 \chi_{0000100}+7 \chi_{1000001}+4 \chi_{0100000}+4 \chi_{0000001} \\
& z_{1} z_{3} z_{7}=\chi_{1010001}+\chi_{0001001}+\chi_{2100000}+\chi_{1000011}+2 \chi_{0110000}+\chi_{0100002}+3 \chi_{1000100}+3 \chi_{2000001} \\
& +3 \chi_{0100010}+5 \chi_{0010001}+3 \chi_{0000011}+6 \chi_{1100000}+5 \chi_{0000100}+6 \chi_{1000001}+4 \chi_{0100000}+2 \chi_{0000001} \\
& z_{2} z_{3} z_{7}=\chi_{0110001}+\chi_{1000101}+\chi_{0020000}+\chi_{0100011}+\chi_{2000002}+\chi_{1200000}+\chi_{0010002}+2 \chi_{1001000} \\
& +3 \chi_{0100100}+2 \chi_{2000010}+\chi_{0000012}+4 \chi_{0010010}+7 \chi_{1100001}+2 \chi_{0000020}+4 \chi_{0200000}+5 \chi_{0000101} \\
& +5 \chi_{1000002}+\chi_{3000000}+6 \chi_{1010000}+7 \chi_{0001000}+10 \chi_{1000010}+5 \chi_{2000000}+9 \chi_{0100001}+7 \chi_{0010000} \\
& +4 \chi_{0000002}+6 \chi_{0000010}+4 \chi_{1000000}+\chi_{0000000} \\
& z_{3}^{2} z_{7}=\chi_{0020001}+\chi_{1001001}+\chi_{0100101}+2 \chi_{1110000}+\chi_{2000011}+\chi_{0010011}+2 \chi_{0101000}+2 \chi_{1100002} \\
& +2 \chi_{2000100}+\chi_{0000021}+4 \chi_{0010100}+\chi_{0000102}+6 \chi_{1100010}+4 \chi_{0200001}+2 \chi_{3000001}+4 \chi_{0000110} \\
& +\chi_{1000003}+8 \chi_{1010001}+7 \chi_{2100000}+8 \chi_{0001001}+11 \chi_{0110000}+10 \chi_{1000011}+14 \chi_{1000100}+6 \chi_{0100002} \\
& +2 \chi_{0000003}+12 \chi_{2000001}+14 \chi_{0100010}+16 \chi_{0010001}+11 \chi_{0000011}+16 \chi_{1100000}+12 \chi_{0000100}+14 \chi_{1000001} \\
& +7 \chi_{0100000}+5 \chi_{0000001} \\
& z_{1} z_{4} z_{7}=\chi_{1001001}+\chi_{0100101}+\chi_{1110000}+\chi_{0010011}+2 \chi_{0101000}+\chi_{1100002}+\chi_{2000100}+3 \chi_{0010100}
\end{aligned}
$$




$$
\begin{aligned}
& +\chi_{0000102}+4 \chi_{1100010}+3 \chi_{0200001}+3 \chi_{0000110}+4 \chi_{1010001}+6 \chi_{0001001}+4 \chi_{1000011}+3 \chi_{2100000} \\
& +7 \chi_{0110000}+3 \chi_{0100002}+8 \chi_{1000100}+3 \chi_{2000001}+8 \chi_{0100010}+8 \chi_{0010001}+7 \chi_{1100000}+3 \chi_{0000011} \\
& +5 \chi_{0000100}+3 \chi_{1000001}+2 \chi_{0100000} \\
& z_{2} z_{4} z_{7}=\chi_{0101001}+\chi_{0010101}+\chi_{0210000}+\chi_{1100011}+2 \chi_{0011000}+\chi_{0200002}+\chi_{1010002}+3 \chi_{1100100} \\
& +\chi_{0000111}+2 \chi_{0000200}+3 \chi_{1010010}+\chi_{0001002}+3 \chi_{0200010}+\chi_{1000012}+\chi_{0100003}+5 \chi_{0001010} \\
& +3 \chi_{2100001}+3 \chi_{1000020}+8 \chi_{0110001}+2 \chi_{2010000}+6 \chi_{1200000}+8 \chi_{1000101}+3 \chi_{2000002}+4 \chi_{0020000} \\
& +8 \chi_{0100011}+10 \chi_{1001000}+7 \chi_{2000010}+12 \chi_{0100100}+6 \chi_{0010002}+14 \chi_{0010010}+18 \chi_{1100001}+3 \chi_{0000012} \\
& +5 \chi_{0000020}+12 \chi_{0000101}+2 \chi_{3000000}+7 \chi_{0200000}+11 \chi_{1010000}+7 \chi_{1000002}+12 \chi_{0001000}+13 \chi_{1000010} \\
& +4 \chi_{2000000}+11 \chi_{0100001}+2 \chi_{0000002}+7 \chi_{0010000}+4 \chi_{0000010}+2 \chi_{1000000} \\
& z_{3} z_{4} z_{7}=\chi_{0011001}+\chi_{1100101}+\chi_{0200011}+\chi_{0120000}+\chi_{1010011}+2 \chi_{1101000}+\chi_{0000201}+3 \chi_{1010100} \\
& +\chi_{0001011}+\chi_{1000021}+\chi_{2100002}+2 \chi_{0110002}+2 \chi_{0200100}+4 \chi_{0001100}+3 \chi_{2100010}+2 \chi_{1000102} \\
& +7 \chi_{0110010}+3 \chi_{2010001}+7 \chi_{1200001}+2 \chi_{0100012}+\chi_{2000003}+5 \chi_{0020001}+3 \chi_{0300000}+7 \chi_{1000110} \\
& +\chi_{0010003}+12 \chi_{1001001}+8 \chi_{2000011}+5 \chi_{0100020}+\chi_{0000013}+13 \chi_{0100101}+2 \chi_{3100000}+14 \chi_{0010011} \\
& +13 \chi_{1110000}+5 \chi_{0000021}+11 \chi_{2000100}+14 \chi_{1100002}+14 \chi_{0101000}+20 \chi_{0010100}+31 \chi_{1100010}+9 \chi_{0000102} \\
& +18 \chi_{0000110}+6 \chi_{3000001}+27 \chi_{1010001}+5 \chi_{1000003}+17 \chi_{0200001}+29 \chi_{0001001}+18 \chi_{2100000}+29 \chi_{1000011} \\
& +28 \chi_{0110000}+32 \chi_{1000100}+19 \chi_{2000001}+17 \chi_{0100002}+4 \chi_{0000003}+30 \chi_{0100010}+30 \chi_{0010001}+16 \chi_{0000011} \\
& +23 \chi_{1100000}+16 \chi_{0000100}+15 \chi_{1000001}+7 \chi_{0100000}+3 \chi_{0000001} \\
& z_{4}^{2} z_{7}=\chi_{0002001}+\chi_{0110101}+\chi_{0020011}+\chi_{1000201}+\chi_{1200011}+\chi_{0300002}+2 \chi_{0111000}+\chi_{1001011} \\
& +2 \chi_{0020100}+2 \chi_{1200100}+2 \chi_{0100111}+2 \chi_{0300010}+2 \chi_{1110002}+2 \chi_{0101002}+4 \chi_{1001100}+6 \chi_{1110010} \\
& +\chi_{2000021}+\chi_{0010021}+4 \chi_{0100200}+8 \chi_{0101010}+4 \chi_{1020001}+\chi_{2000102}+3 \chi_{0010102}+4 \chi_{2000110} \\
& +4 \chi_{1100012}+\chi_{3000003}+\chi_{0000031}+4 \chi_{2200001}+10 \chi_{0010110}+2 \chi_{0000112}+10 \chi_{1100020} \\
& +6 \chi_{0000120}+6 \chi_{2001001}+2 \chi_{0200003}+10 \chi_{0210001}+6 \chi_{2110000}+2 \chi_{1010003}+14 \chi_{0011001} \\
& +24 \chi_{1100101}+6 \chi_{1300000}+2 \chi_{0001003}+11 \chi_{0120000}+16 \chi_{0200011}+4 \chi_{3000011}+23 \chi_{1101000} \\
& +10 \chi_{0000201}+20 \chi_{0200100}+3 \chi_{1000013}+22 \chi_{1010011}+24 \chi_{0001011}+16 \chi_{2100002}+4 \chi_{3000100}+\chi_{0100004} \\
& +28 \chi_{1010100}+29 \chi_{0001100}+32 \chi_{2100010}+16 \chi_{1000021}+26 \chi_{0110002}+26 \chi_{1000102}+56 \chi_{0110010}+10 \chi_{2000003} \\
& +22 \chi_{0100012}+14 \chi_{0010003}+3 \chi_{4000001}+47 \chi_{1200001}+50 \chi_{1000110}+25 \chi_{2010001}+33 \chi_{0100020}+31 \chi_{0020001} \\
& +73 \chi_{1001001}+14 \chi_{0300000}+50 \chi_{2000011}+14 \chi_{3100000}+60 \chi_{1110000}+70 \chi_{0100101}+\chi_{0000005}+75 \chi_{0010011} \\
& +8 \chi_{0000013}+25 \chi_{0000021}+50 \chi_{2000100}+26 \chi_{3000001}+68 \chi_{1100002}+41 \chi_{0000102}+56 \chi_{0101000}+74 \chi_{0010100} \\
& +114 \chi_{1100010}+24 \chi_{1000003}+52 \chi_{0200001}+92 \chi_{1010001}+60 \chi_{0000110}+92 \chi_{0001001}+51 \chi_{2100000}+90 \chi_{1000011} \\
& +48 \chi_{0100002}+72 \chi_{0110000}+81 \chi_{1000100}+12 \chi_{0000003}+70 \chi_{0100010}+48 \chi_{2000001}+68 \chi_{0010001}+46 \chi_{1100000} \\
& +37 \chi_{0000011}+32 \chi_{0000100}+30 \chi_{1000001}+12 \chi_{0100000}+7 \chi_{0000001} \\
& z_{1} z_{5} z_{7}=\chi_{1000101}+\chi_{0100011}+\chi_{1001000}+2 \chi_{0100100}+\chi_{0010002}+\chi_{2000010}+3 \chi_{0010010}+\chi_{0000012} \\
& +2 \chi_{0000020}+4 \chi_{1100001}+5 \chi_{0000101}+2 \chi_{0200000}+3 \chi_{1000002}+3 \chi_{1010000}+5 \chi_{0001000}+7 \chi_{1000010} \\
& +7 \chi_{0100001}+2 \chi_{0000002}+2 \chi_{2000000}+5 \chi_{0010000}+4 \chi_{0000010}+2 \chi_{1000000} \\
& z_{2} z_{5} z_{7}=\chi_{0100101}+\chi_{0010011}+\chi_{0000021}+\chi_{0101000}+\chi_{1100002}+2 \chi_{0010100}+\chi_{0000102}+3 \chi_{1100010} \\
& +3 \chi_{1010001}+4 \chi_{0000110}+\chi_{1000003}+3 \chi_{0200001}+5 \chi_{0001001}+2 \chi_{2100000}+7 \chi_{1000011}+6 \chi_{0110000} \\
& +5 \chi_{0100002}+8 \chi_{1000100}+2 \chi_{0000003}+6 \chi_{2000001}+11 \chi_{0100010}+11 \chi_{0010001}+9 \chi_{0000011}+10 \chi_{1100000} \\
& +8 \chi_{0000100}+10 \chi_{1000001}+5 \chi_{0100000}+3 \chi_{0000001} \\
& z_{3} z_{5} z_{7}=\chi_{0010101}+\chi_{1100011}+\chi_{0011000}+\chi_{0200002}+\chi_{0000111}+\chi_{1010002}+\chi_{0001002}+2 \chi_{1100100}+2 \chi_{1000012} \\
& +3 \chi_{1010010}+2 \chi_{0000200}+2 \chi_{0200010}+4 \chi_{0001010}+4 \chi_{1000020}+\chi_{0100003}+3 \chi_{2100001}+\chi_{0000004} \\
& +7 \chi_{0110001}+9 \chi_{1000101}+2 \chi_{2010000}+5 \chi_{1200000}+5 \chi_{2000002}+4 \chi_{0020000}+10 \chi_{0100011}+9 \chi_{1001000} \\
& +9 \chi_{2000010}+12 \chi_{0100100}+8 \chi_{0010002}+17 \chi_{0010010}+23 \chi_{1100001}+3 \chi_{3000000}+9 \chi_{0200000}+6 \chi_{0000012} \\
& +8 \chi_{0000020}+17 \chi_{0000101}+14 \chi_{1000002}+14 \chi_{1010000}+16 \chi_{0001000}+22 \chi_{1000010}+8 \chi_{2000000}+18 \chi_{0100001} \\
& +7 \chi_{0000002}+12 \chi_{0010000}+9 \chi_{0000010}+5 \chi_{1000000}+\chi_{0000000} \\
& z_{4} z_{5} z_{7}=\chi_{0001101}+\chi_{0110011}+\chi_{1000111}+\chi_{0002000}+\chi_{0020002}+\chi_{0100021}+\chi_{1200002}+2 \chi_{0110100} \\
& +\chi_{1001002}+2 \chi_{0020010}+2 \chi_{1000200}+2 \chi_{0100102}+2 \chi_{1200010}+4 \chi_{1001010}+6 \chi_{0100110}+\chi_{2000012}
\end{aligned}
$$




$$
\begin{aligned}
& +6 \chi_{1110001}+2 \chi_{0300001}+2 \chi_{0010012}+8 \chi_{0101001}+3 \chi_{1020000}+2 \chi_{1100003}+2 \chi_{2000020}+\chi_{0000022} \\
& +\chi_{0000103}+5 \chi_{0010020}+4 \chi_{2000101}+12 \chi_{0010101}+2 \chi_{0000030}+17 \chi_{1100011}+3 \chi_{2200000}+4 \chi_{2001000} \\
& +7 \chi_{0210000}+11 \chi_{0000111}+8 \chi_{0200002}+2 \chi_{3000002}+11 \chi_{0011000}+20 \chi_{1100100}+\chi_{1000004}+11 \chi_{1010002} \\
& +13 \chi_{0001002}+16 \chi_{0200010}+10 \chi_{0000200}+3 \chi_{3000010}+22 \chi_{1010010}+27 \chi_{0001010}+13 \chi_{1000012}+21 \chi_{2100001} \\
& +7 \chi_{0100003}+39 \chi_{0110001}+\chi_{4000000}+19 \chi_{1000020}+41 \chi_{1000101}+2 \chi_{0000004}+22 \chi_{1200000}+38 \chi_{0100011} \\
& +18 \chi_{2000002}+11 \chi_{2010000}+15 \chi_{0020000}+36 \chi_{1001000}+28 \chi_{0010002}+38 \chi_{0100100}+15 \chi_{0000012}+29 \chi_{2000010} \\
& +8 \chi_{3000000}+47 \chi_{0010010}+58 \chi_{1100001}+17 \chi_{0000020}+30 \chi_{1010000}+17 \chi_{0200000}+38 \chi_{0000101}+25 \chi_{1000002} \\
& +32 \chi_{0001000}+37 \chi_{1000010}+27 \chi_{0100001}+11 \chi_{2000000}+16 \chi_{0010000}+8 \chi_{0000002}+11 \chi_{0000010}+5 \chi_{1000000} \\
& +\chi_{0000000} \\
& z_{5}^{2} z_{7}=\chi_{0000201}+\chi_{0001011}+\chi_{0110002}+2 \chi_{0001100}+\chi_{1000021}+2 \chi_{0110010}+\chi_{1000102}+2 \chi_{0100012} \\
& +2 \chi_{0020001}+4 \chi_{1000110}+\chi_{2000003}+2 \chi_{1200001}+4 \chi_{0100020}+4 \chi_{1001001}+8 \chi_{0100101}+\chi_{0010003} \\
& +4 \chi_{1110000}+4 \chi_{2000011}+\chi_{0000013}+10 \chi_{0010011}+\chi_{0300000}+6 \chi_{0000021}+6 \chi_{0101000}+3 \chi_{2000100} \\
& +10 \chi_{1100002}+11 \chi_{0010100}+8 \chi_{0000102}+18 \chi_{1100010}+3 \chi_{3000001}+12 \chi_{0200001}+16 \chi_{0000110}+6 \chi_{1000003} \\
& +16 \chi_{1010001}+22 \chi_{0001001}+10 \chi_{2100000}+20 \chi_{0110000}+27 \chi_{1000011}+17 \chi_{0100002}+24 \chi_{1000100}+28 \chi_{0100010} \\
& +18 \chi_{2000001}+28 \chi_{0010001}+6 \chi_{0000003}+22 \chi_{1100000}+20 \chi_{0000011}+17 \chi_{0000100}+19 \chi_{1000001}+8 \chi_{0100000} \\
& +6 \chi_{0000001} \\
& z_{1} z_{6} z_{7}=\chi_{1000011}+\chi_{0100002}+\chi_{0000003}+\chi_{1000100}+2 \chi_{0100010}+\chi_{2000001}+3 \chi_{0010001}+3 \chi_{1100000} \\
& +4 \chi_{0000011}+4 \chi_{0000100}+6 \chi_{1000001}+4 \chi_{0100000}+3 \chi_{0000001} \\
& z_{2} z_{6} z_{7}=\chi_{0100011}+\chi_{0010002}+\chi_{0100100}+\chi_{0000012}+2 \chi_{0010010}+2 \chi_{0000020}+3 \chi_{1100001}+2 \chi_{0200000} \\
& +2 \chi_{1010000}+4 \chi_{0000101}+4 \chi_{0001000}+4 \chi_{1000002}+7 \chi_{1000010}+8 \chi_{0100001}+4 \chi_{0000002}+3 \chi_{2000000} \\
& +6 \chi_{0010000}+6 \chi_{0000010}+4 \chi_{1000000}+\chi_{0000000} \\
& z_{3} z_{6} z_{7}=\chi_{0010011}+\chi_{1100002}+\chi_{0010100}+\chi_{0000102}+2 \chi_{1100010}+3 \chi_{1010001}+2 \chi_{0000110}+\chi_{1000003} \\
& +2 \chi_{0200001}+4 \chi_{0001001}+6 \chi_{1000011}+2 \chi_{2100000}+5 \chi_{0110000}+5 \chi_{0100002}+7 \chi_{1000100}+2 \chi_{0000003} \\
& +6 \chi_{2000001}+9 \chi_{0100010}+12 \chi_{0010001}+8 \chi_{0000011}+11 \chi_{1100000}+9 \chi_{0000100}+11 \chi_{1000001}+6 \chi_{0100000} \\
& +3 \chi_{0000001} \\
& z_{4} z_{6} z_{7}=\chi_{0001011}+\chi_{0110002}+\chi_{0001100}+0 \chi_{1000021}+2 \chi_{0110010}+\chi_{1000102}+\chi_{0100012}+2 \chi_{0020001} \\
& +2 \chi_{1000110}+2 \chi_{1200001}+2 \chi_{0100020}+4 \chi_{1001001}+6 \chi_{0100101}+\chi_{0010003}+4 \chi_{1110000}+2 \chi_{2000011} \\
& +7 \chi_{0010011}+\chi_{0300000}+2 \chi_{0000021}+6 \chi_{0101000}+3 \chi_{2000100}+7 \chi_{1100002}+9 \chi_{0010100}+5 \chi_{0000102} \\
& +14 \chi_{1100010}+\chi_{3000001}+9 \chi_{0200001}+10 \chi_{0000110}+2 \chi_{1000003}+13 \chi_{1010001}+18 \chi_{0001001}+8 \chi_{2100000} \\
& +17 \chi_{0110000}+15 \chi_{1000011}+10 \chi_{0100002}+19 \chi_{1000100}+19 \chi_{0100010}+10 \chi_{2000001}+19 \chi_{0010001}+\chi_{0000003} \\
& +15 \chi_{1100000}+9 \chi_{0000011}+11 \chi_{0000100}+8 \chi_{1000001}+4 \chi_{0100000}+\chi_{0000001} \\
& z_{5} z_{6} z_{7}=\chi_{0000111}+\chi_{0001002}+\chi_{0000200}+\chi_{1000012}+2 \chi_{0001010}+2 \chi_{0110001}+\chi_{0100003}+\chi_{0020000} \\
& +2 \chi_{1000020}+4 \chi_{1000101}+6 \chi_{0100011}+2 \chi_{2000002}+5 \chi_{0010002}+\chi_{1200000}+3 \chi_{1001000}+6 \chi_{0100100} \\
& +3 \chi_{2000010}+9 \chi_{0010010}+4 \chi_{0000012}+12 \chi_{1100001}+6 \chi_{0000020}+5 \chi_{0200000}+13 \chi_{0000101}+9 \chi_{1000002} \\
& +\chi_{3000000}+7 \chi_{1010000}+11 \chi_{0001000}+15 \chi_{1000010}+14 \chi_{0100001}+5 \chi_{0000002}+5 \chi_{2000000}+9 \chi_{0010000} \\
& +8 \chi_{0000010}+4 \chi_{1000000}+\chi_{0000000} \\
& z_{6}^{2} z_{7}=\chi_{0000021}+\chi_{0000102}+2 \chi_{0000110}+2 \chi_{0001001}+\chi_{1000003}+\chi_{0110000}+4 \chi_{1000011}+4 \chi_{0100002} \\
& +3 \chi_{1000100}+2 \chi_{0000003}+6 \chi_{0100010}+3 \chi_{2000001}+7 \chi_{0010001}+6 \chi_{1100000}+9 \chi_{0000011}+8 \chi_{0000100} \\
& +10 \chi_{1000001}+6 \chi_{0100000}+5 \chi_{0000001} \\
& z_{1} z_{7}^{2}=\chi_{1000002}+\chi_{1000010}+2 \chi_{0100001}+\chi_{2000000}+2 \chi_{0000002}+2 \chi_{0010000}+3 \chi_{0000010}+3 \chi_{1000000} \\
& +\chi_{0000000} \\
& z_{2} z_{7}^{2}=\chi_{0100002}+\chi_{0100010}+2 \chi_{0010001}+2 \chi_{0000011}+2 \chi_{1100000}+3 \chi_{0000100}+4 \chi_{1000001}+4 \chi_{0100000} \\
& +2 \chi_{0000001} \\
& z_{3} z_{7}^{2}=\chi_{0010002}+\chi_{0010010}+2 \chi_{1100001}+\chi_{0200000}+2 \chi_{1010000}+2 \chi_{0000101}+3 \chi_{0001000}+2 \chi_{1000002} \\
& +4 \chi_{1000010}+5 \chi_{0100001}+\chi_{0000002}+2 \chi_{2000000}+5 \chi_{0010000}+3 \chi_{0000010}+2 \chi_{1000000} \\
& z_{4} z_{7}^{2}=\chi_{0001002}+\chi_{0001010}+2 \chi_{0110001}+\chi_{0020000}+2 \chi_{1000101}+2 \chi_{0100011}+2 \chi_{0010002}+\chi_{1200000}
\end{aligned}
$$




$$
\begin{aligned}
& +3 \chi_{1001000}+4 \chi_{0100100}+\chi_{2000010}+5 \chi_{0010010}+6 \chi_{1100001}+\chi_{0000020}+3 \chi_{0200000}+5 \chi_{0000101} \\
& +\chi_{1000002}+4 \chi_{1010000}+7 \chi_{0001000}+5 \chi_{1000010}+4 \chi_{0100001}+\chi_{2000000}+3 \chi_{0010000}+\chi_{0000010} \\
z_{5} z_{7}^{2} & =\chi_{0000102}+\chi_{0000110}+2 \chi_{0001001}+\chi_{0110000}+2 \chi_{1000011}+2 \chi_{0100002}+3 \chi_{1000100}+4 \chi_{0100010} \\
& +\chi_{2000001}+5 \chi_{0010001}+4 \chi_{1100000}+4 \chi_{0000011}+6 \chi_{0000100}+4 \chi_{1000001}+3 \chi_{0100000}+\chi_{0000001} \\
z_{6} z_{7}^{2} & =\chi_{0000012}+\chi_{0000020}+2 \chi_{0000101}+\chi_{0001000}+2 \chi_{1000002}+3 \chi_{1000010}+4 \chi_{0100001}+\chi_{2000000} \\
& +3 \chi_{0000002}+3 \chi_{0010000}+5 \chi_{0000010}+3 \chi_{1000000}+\chi_{0000000} \\
z_{7}^{3} & =\chi_{0000003}+2 \chi_{0000011}+\chi_{0000100}+3 \chi_{1000001}+2 \chi_{0100000}+4 \chi_{0000001}
\end{aligned}
$$

\section{References}

[1] Calogero F., Classical Many Body Problems Amenable to Exact Treatments, Springer, 2001.

[2] Perelomov A.M., Integrable Systems of Classical Mechanics and Lie Algebras, Birkhäuser, 1990.

[3] Calogero F., J. Math. Phys. 12, 419-436, 1971.

[4] Sutherland B., Phys. Rev. A4, 2019-2021, 1972.

[5] Moser J., Adv. Math. 16, 197-220, 1975.

[6] Olshanetsky M.A. and Perelomov A.M., Invent. Math. 37, 93-108, 1976.

[7] Olshanetsky M.A. and Perelomov A.M., Lett. Math. Phys. 2, 7-13, 1977.

[8] Olshanetsky M.A. and Perelomov A.M., Funct. Anal. Appl. 12, 121-128, 1978.

[9] Olshanetsky M.A. and Perelomov A.M., Phys. Rep. 71, 314-400, 1981.

[10] Olshanetsky M.A. and Perelomov A.M., Phys. Rep. 94, 313-404, 1983.

[11] van Diejen J.F and Vinet L. (Eds.), Calogero-Moser-Sutherland Models, Springer, 2000.

[12] Caselle M., Nuclear Phys. Proc. Suppl. 45A, 120-129, 1996.

[13] Capello M. and Caselle M., J. Phys, C15, 6845-6854, 2003.

[14] Perelomov A.M., J. Phys. A31, L31-L37, 1998.

[15] Perelomov A.M., Ragoucy E. and Zaugg Ph., J. Phys. A31, L559-L565, 1998.

[16] Perelomov A.M., J. Phys. A32, 8563-8576, 1999.

[17] García Fuertes W., Lorente M., Perelomov A.M., J. Phys. A34, 10963-10973, 2001.

[18] García Fuertes W., Perelomov A.M., Theor. Math. Phys., 131, 609-611, 2002; math-ph/0201026.

[19] Fernández Núñez J., García Fuertes W., Perelomov A.M., Phys. Lett. A307, 233-238, 2003.

[20] Fernández Núñez J., García Fuertes W., Perelomov A.M., J. Math. Phys. 44, 4957-4974, 2003.

[21] Fernández-Núñez J., García-Fuertes W., Perelomov A.M., J. Nonlinear Math. Phys. 12, Suppl. 1, 280-301, 2005.

[22] Onishchik A.L. and Vinberg E.B., Lie Groups and Algebraic Groups, Springer, 1990. 
[23] Cornwell J.F., Group Theory in Physics, vol II,, Academic Press, 1984; Bourbaki N. Groupes et Algebres de Lie, Hermann, 1975.

[24] Baez J.C., Bull. Amer. Math. Soc. 39, 145-205, 2002.

[25] Khastgir S.P., Pocklington A.J., Sasaki R., J. Phys. A33, 9033-9064, 2000.

[26] Slansky R., Phys. Rep. 79, 1-128, 1981.

[27] Fernández Núñez J., García Fuertes W., Perelomov A.M., J. Math. Phys. 46, 073508, 2005.

[28] King R.C. and Wybourne B.G., J. Phys. A35, 3489-3513, 2002. 